Kent Andersen · Gérard Cornuéjols · Yanjun Li

\title{
Split closure and intersection cuts $^{\star}$
}

Received: February 4, 2003 / Accepted: September 1, 2004

Published online: December 29, 2004 - @ S Springer-Verlag 2004

\begin{abstract}
In the seventies, Balas introduced intersection cuts for a Mixed Integer Linear Program (MILP), and showed that these cuts can be obtained by a closed form formula from a basis of the standard linear programming relaxation. In the early nineties, Cook, Kannan and Schrijver introduced the split closure of a MILP, and showed that the split closure is a polyhedron. In this paper, we show that the split closure can be obtained using only intersection cuts. We give two different proofs of this result, one geometric and one algebraic. The result is then used to provide a new proof of the fact that the split closure of a MILP is a polyhedron. Finally, we extend the result to more general disjunctions.
\end{abstract}

\section{Introduction}

In the seventies, Balas [2] showed that cuts for a Mixed Integer Linear Program (MILP) can be derived from disjunctions applied to the bases of its linear programming relaxation. In that paper, the basis was an optimal basis to the linear programming relaxation. The cut was obtained by a closed form formula and was called the intersection cut.

Later in the seventies, Balas [3] generalized his results to polyhedra. He proved that, given a polyhedron $P$ and a disjunction, a cut for a point $\bar{x} \in P$ that violates the disjunction can be obtained by solving a linear program. The idea was further expanded in the early nineties when Cook, Kannan and Schrijver [6] studied split cuts obtained from disjunctions with two terms called split disjunctions. The intersection of all split cuts is called the split closure of a MILP. Cook, Kannan and Schrijver proved that the split closure of a MILP is a polyhedron. Split cuts are equivalent to Gomory mixed integer cuts [9] and to mixed integer rounding cuts generated from linear combinations of constraints $[10,8]$. Relations between these cuts and other families of cuts are surveyed in [7].

Intersection cuts are a special type of split cuts. The main contribution of this paper is to show that, conversely, the split closure of a MILP can be obtained using only intersection cuts, provided infeasible and non-optimal bases are considered. This result was first shown by Balas and Perregaard [4] for mixed 0-1 linear programs. For such programs, it suffices to consider $0-1$ disjunctions of the form $x_{j} \leq 0$ or $x_{j} \geq 1$. We generalize their

K. Andersen: Tepper School of Business, Carnegie Mellon University, Pittsburgh, PA 15213, USA. e-mail: kha@andrew.cmu.edu

G. Cornuéjols: Tepper School of Business, Carnegie Mellon University, Pittsburgh, PA 15213, USA; and LIF, University of Marseille at Luminy, 13288 Marseille, France. e-mail: gc0v@andrew . cmu . edu

Y. Li: Krannert Graduate School of Management, Purdue University, West Lafayette, IN 47907, USA. e-mail: li14@mgmt.purdue.edu

* Supported by NSF grant DMI-0352885 and ONR grant N00014-97-1-0196. 
result from 0-1 disjunctions to more general two-term disjunctions. We also consider the more general setting of MILPs. Two different proofs are provided, one geometric and one algebraic. A consequence is a new proof of the fact that the split closure of a MILP is a polyhedron.

Our main result shows that the only interesting split cuts are intersection cuts. This result has algorithmic implications since intersection cuts can be obtained by a closed form formula and many of the cutting planes used in practice are split cuts. In particular, Gomory mixed integer cuts generated from an optimal basis are currently the most effective in commercial codes [5]. In [1], we exploit the main result of this paper by fixing the optimal basis and modifying the split disjunctions that generate the Gomory mixed integer cuts. On several test problems, these cuts drastically reduce the number of nodes in a branch-and-cut framework.

\subsection{Notation}

We consider the Mixed Integer Linear Program

$$
\text { (MILP) } \min \left\{c^{T} x: A x \leq b, x_{j} \text { integer, } j \in N_{I}\right\},
$$

where $c \in \mathbb{R}^{n}, b \in \mathbb{R}^{m}, A \in \mathbb{R}^{m \times n}$ and $N_{I} \subseteq N:=\{1,2, \ldots, n\}$. Let LP be the Linear Programming problem obtained from MILP by dropping the integrality conditions on $x_{j}$ for $j \in N_{I} . P_{I}$ and $P$ denote the sets of feasible solutions to MILP and LP respectively. The set $M:=\{1,2, \ldots, m\}$ is used to index the rows of $A$. Given $i \in M$, the $i^{t h}$ row of $A$ is denoted $a_{i}$. We assume $a_{i} \neq 0_{n}$ for all $i \in M$. A basis of $A$ is an $n$-subset $B$ of $M$, such that the vectors $\left\{a_{i}\right\}_{i \in B}$ are linearly independent. Observe that, if the rank of $A$ is less than $n, A$ does not have bases.

The relaxations of $P$ obtained by dropping constraints of $P$ play a major role in this paper. Given $S \subseteq M$, define

$$
P(S):=\left\{x \in \mathbb{R}^{n}: a_{i .}^{T} x \leq b_{i}, \forall i \in S\right\}
$$

to be the relaxation of $P$ obtained by keeping the constraints in $S$ only. The rank of the sub-matrix of $A$ induced by the rows in $S$ is denoted $r(S)$. The rank of $A$ is denoted simply by $r:=r(M)$.

The case where the vectors $\left\{a_{i}\right\}_{i \in S}$ are linearly independent is especially important. Given a positive integer $k$, let

$$
\mathcal{B}_{k}^{*}:=\{S \subseteq M:|S|=k \text { and } r(S)=k\}
$$

denote the set of $k$-subsets $S$ of $M$, such that the vectors $\left\{a_{i}\right\}_{i \in S}$ are linearly independent $\left(\mathcal{B}_{n}^{*}\right.$ denotes the set of bases of $A$ ). Note that $\mathcal{B}_{k}^{*}$ is empty if $k>r$. Given $B \in \mathcal{B}_{k}^{*}$ where $k \leq r$, the set $P(B)$ is a translate of a polyhedral cone. Specifically, $P(B)$ can be written as $P(B)=C+\bar{x}(B)$, where $\bar{x}(B)$ solves the system $a_{i}^{T} x=b_{i}, \forall i \in B$, and $C$ is the polyhedral cone $C:=\left\{x \in \mathbb{R}^{n}: a_{i}^{T} x \leq 0, \forall i \in B\right\}$. Translates of polyhedral cones have many properties in common with polyhedral cones. We call them conic polyhedra in the remainder of this paper. 
The most general two-term disjunction considered in this paper is an expression $D$ of the form $D^{1} x \leq d^{1} \vee D^{2} x \leq d^{2}$, where $D^{1} \in \mathbb{R}^{m_{1} \times n}, D^{2} \in \mathbb{R}^{m_{2} \times n}, d^{1} \in \mathbb{R}^{m_{1}}$ and $d^{2} \in \mathbb{R}^{m_{2}}$. The set of points in $\mathbb{R}^{n}$ satisfying the disjunction $D$ is denoted by $F_{D}$. The convex hull of $P \cap F_{D}$, denoted by $\operatorname{Conv}\left(P \cap F_{D}\right)$, is called the disjunctive hull defined by $P$ and $D$ in the remainder of this paper. In addition, given a subset $S$ of the constraints, the set $\operatorname{Conv}\left(P(S) \cap F_{D}\right)$ is called the disjunctive hull defined by $S$ and $D$. In particular, given a set $B \in \mathcal{B}_{r}^{*}$, the set $\operatorname{Conv}\left(P(B) \cap F_{D}\right)$ is called a basic disjunctive hull. An important two-term disjunction is the split disjunction $D\left(\pi, \pi_{0}\right)$ of the form $\pi^{T} x \leq \pi_{0} \vee \pi^{T} x \geq \pi_{0}+1$, where $\left(\pi, \pi_{0}\right) \in \mathbb{Z}^{n+1}$ and $\pi_{j}=0$ for all $j \notin N_{I}$.

Let $\Pi^{n}\left(N_{I}\right):=\left\{\left(\pi, \pi_{0}\right) \in \mathbb{Z}^{n+1}: \pi_{j}=0, j \notin N_{I}\right\}$. The split closure of a MILP, denoted by SC, is defined to be the intersection of the disjunctive hulls defined by $P$ and $D\left(\pi, \pi_{0}\right)$ over all the disjunctions $\left(\pi, \pi_{0}\right)$ in $\Pi^{n}\left(N_{I}\right)$, i.e.,

$$
\mathrm{SC}:=\cap_{\left(\pi, \pi_{0}\right) \in \Pi^{n}\left(N_{I}\right)} \operatorname{Conv}\left(P \cap F_{D\left(\pi, \pi_{0}\right)}\right) .
$$

Similarly, given $S \subseteq M, \operatorname{SC}(S)$ is defined to be the intersection of the disjunctive hulls defined by $P(S)$ and $D\left(\pi, \pi_{0}\right)$ over all disjunctions $\left(\pi, \pi_{0}\right)$ in $\Pi^{n}\left(N_{I}\right)$. A split cut is a valid inequality for SC.

Several results presented in this paper about split disjunctions generalize to disjunctions $D\left(\pi, \pi_{0}^{1}, \pi_{0}^{2}\right)$ of the form $\pi^{T} x \leq \pi_{0}^{1} \vee \pi^{T} x \geq \pi_{0}^{2}$, where $\pi \in \mathbb{R}^{n}$ and $\pi_{0}^{1}<\pi_{0}^{2}$. These disjunctions are called general split disjunctions in the remainder of the paper. Given $\pi_{0}^{1}$ and $\pi_{0}^{2}$ satisfying $\pi_{0}^{1}<\pi_{0}^{2}$, not all general split disjunctions are valid for MILP, i.e., MILP may have feasible solutions that violate a disjunction $D\left(\pi, \pi_{0}^{1}, \pi_{0}^{2}\right)$ for some $\pi \in \mathbb{R}^{n}$. However, for the sake of generality, we state and prove some results in terms of general split disjunctions. The results obtained for general split disjunctions imply the corresponding results for split disjunctions.

\subsection{Main Contributions}

The first contribution of this paper is a result (Corollary 1) stating that the split closure of MILP can be written as the intersection of the split closures of the sets $P(B)$ over all sets $B \in \mathcal{B}_{r}^{*}$, i.e., $\mathrm{SC}=\cap_{B \in \mathcal{B}_{r}^{*}} \mathrm{SC}(B)$. We prove this result by proving that the disjunctive hull defined by $P$ and a general split disjunction $D\left(\pi, \pi_{0}^{1}, \pi_{0}^{2}\right)$ can be written as the intersection of the basic disjunctive hulls, i.e., $\operatorname{Conv}\left(P \cap F_{D\left(\pi, \pi_{0}^{1}, \pi_{0}^{2}\right)}\right)=$ $\cap_{B \in \mathcal{B}_{r}^{*}} \operatorname{Conv}\left(P(B) \cap F_{D\left(\pi, \pi_{0}^{1}, \pi_{0}^{2}\right)}\right.$. We provide both a geometric and an algebraic proof of this result. The result implies that the disjunctive hull defined by $P$ and a split disjunction $D\left(\pi, \pi_{0}\right)$ can be obtained using only intersection cuts (A precise definition of intersection cut is given in Sect. 2.1). In turn, this implies that the split closure of a MILP can be obtained using only intersection cuts. Furthermore, the result leads to a new proof of the fact that the split closure is a polyhedron (Theorem 2).

The second contribution of this paper is Theorem 3 that describes the disjunctive hull defined by $P$ and a general two-term disjunction $D$.

The rest of this paper is organized as follows. In Sect. 2, we give an outline of the main results in the paper. We also give examples showing that some, seemingly natural, extensions of these results are incorrect. In Sect. 3, we consider conic polyhedra. In 


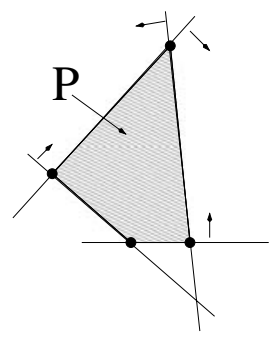

(a) A polytope

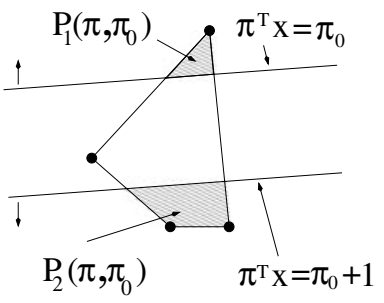

(b) A polytope and a split disjunction

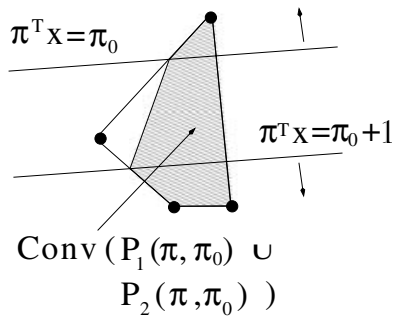

(c) The disjunctive hull

Fig. 1. Effect of applying a split disjunction to a polytope

particular, given $B \in \mathcal{B}_{r}^{*}$, we show that the split closure of the conic polyhedron $P(B)$ is a polyhedron. In Sect. 4 , a characterization of the split closure in terms of intersection cuts is presented, and a geometric argument for the validity of the result is presented. In Sect. 5, we generalize the results of Sect. 4 to more general two-term disjunctions. The arguments used in Sect. 5 are mostly algebraic. In fact, Sect. 4 and Sect. 5 can be read independently.

\section{Outline}

We start by giving an outline and by presenting examples of the results derived in the paper. Furthermore, we give counterexamples of some seemingly natural extensions of these results.

Figure 1(a) gives an example of a polyhedron $P$ (in this case a polytope). In Figure 1(b), a split disjunction $D\left(\pi, \pi_{0}\right)$ is given with $P$. As can be seen from Figure 1(b), $P \cap D\left(\pi, \pi_{0}\right)$ contains two disjoint parts $P_{1}\left(\pi, \pi_{0}\right)$ and $P_{2}\left(\pi, \pi_{0}\right)$ (either might be empty). The main reason that split disjunctions are interesting is that the valid inequalities for $P_{1}\left(\pi, \pi_{0}\right) \cup P_{2}\left(\pi, \pi_{0}\right)$ are valid inequalities for MILP. Since an inequality is valid for $P_{1}\left(\pi, \pi_{0}\right) \cup P_{2}\left(\pi, \pi_{0}\right)$ if and only if it is valid for $\operatorname{Conv}\left(P_{1}\left(\pi, \pi_{0}\right) \cup P_{2}\left(\pi, \pi_{0}\right)\right)$, this leads naturally to a study of the facial structure of the polyhedron $\operatorname{Conv}\left(P_{1}\left(\pi, \pi_{0}\right) \cup\right.$ $\left.P_{2}\left(\pi, \pi_{0}\right)\right)$. The shaded area in Figure 1(c) describes $\operatorname{Conv}\left(P_{1}\left(\pi, \pi_{0}\right) \cup P_{2}\left(\pi, \pi_{0}\right)\right)$.

\subsection{Split disjunctions, conic polyhedra and intersection cuts}

It seems natural to start with the simplest polyhedra. Consider a conic polyhedron $P(B)$ for $B \in \mathcal{B}_{r}^{*}$. Due to the linear independence of the vectors $\left\{a_{i}\right\}_{i \in B}$, this polyhedron is sufficiently simple. Observe that, in the case where $r=n, B$ defines a basis of $A$. Figure 2(a) shows a polyhedron in the two-dimensional plane and gives an example of such a set $B$. As illustrated in Figure 2(b), $P(B)$ is the set obtained from $P$ by ignoring the constraints in $M \backslash B$. 


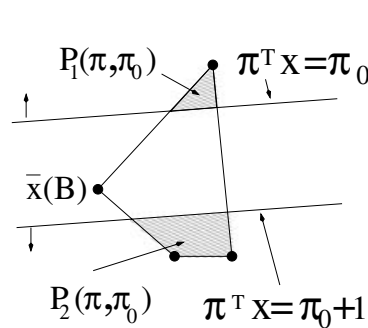

(a) A basic solution

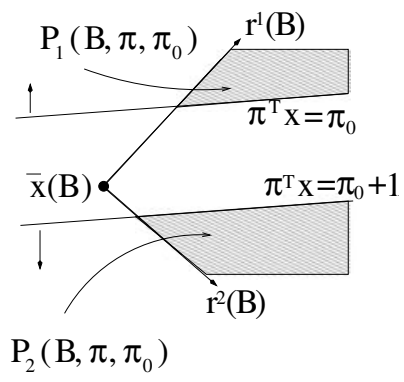

(b) The conic polyhedron

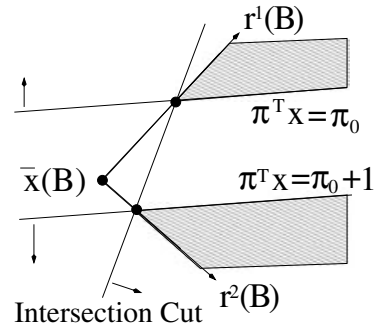

(c) Intersection cut

Fig. 2. Deriving a conic polyhedron and an intersection cut

In Sect. 1, we gave an inequality description of the polyhedron $P(B)$. However, $P(B)$ can also be described in terms of its extreme points and extreme rays as follows. Let $\bar{x}(B)$ satisfy $a_{i}^{T} \bar{x}(B)=b_{i}$ for all $i \in B$. Furthermore, let $L(B):=\left\{x \in \mathbb{R}^{n}\right.$ : $\left.a_{i .}^{T} x=0, \forall i \in B\right\} . L(B)$ is the null space of the matrix $\left[a_{i}\right]_{i \in B}$, and $\bar{x}(B)+L(B)$ is the intersection of the hyperplanes $a_{i}^{T} x=b_{i}$ for $i \in B$. In the example of Figure 2, there are two linearly independent vectors in two dimensions, so $L(B)$ reduces to $\left\{0_{n}\right\}$ and $\bar{x}(B)$ is uniquely defined. If $r<n$, this is not the case. The extreme rays $r^{i}(B)$ of $P(B)$ can be obtained as follows. Since the vectors $\left\{a_{i}\right\}_{i \in B}$ are linearly independent, there exists a solution $r^{i}(B)$ to the system $a_{k}^{T} r^{i}(B)=0, \forall k \in B \backslash\{i\}$, and $a_{i .}^{T} r^{i}(B)=-1$. Now $P(B)$ can be written as

$$
P(B)=\bar{x}(B)+L(B)+\text { Cone }\left(\left\{r^{i}(B)\right\}_{i \in B}\right),
$$

where Cone $\left(\left\{r^{i}(B)\right\}_{i \in B}\right):=\left\{x \in \mathbb{R}^{n}: x=\sum_{i \in B} \lambda_{i} r^{i}(B), \lambda_{i} \geq 0, i \in B\right\}$ denotes the polyhedral cone generated by the vectors $\left\{r^{i}(B)\right\}_{i \in B}$. Observe that the vectors $\left\{r^{i}(B)\right\}_{i \in B}$ are linearly independent.

We are now in position to derive the intersection cut. Let $D\left(\pi, \pi_{0}^{1}, \pi_{0}^{2}\right)$ be a general split disjunction. Assume all points $y$ in $\bar{x}(B)+L(B)$ violate the disjunction $D\left(\pi, \pi_{0}^{1}, \pi_{0}^{2}\right)$ (Lemma 1 below shows this is the only interesting case). This implies that the linear function $\pi^{T} x$ is constant on $\bar{x}(B)+L(B)$. Otherwise, the function value can be made as positive (or negative) as we want by choosing particular points in $\bar{x}(B)+L(B)$, which is a contradiction to the assumption that all points in $\bar{x}(B)+L(B)$ violate the disjunction $D\left(\pi, \pi_{0}^{1}, \pi_{0}^{2}\right)$. Define $\epsilon^{1}\left(\pi, \pi_{0}^{1}, B\right):=\pi^{T} \bar{x}(B)-\pi_{0}^{1}$ and $\epsilon^{2}\left(\pi, \pi_{0}^{2}, B\right):=$ $\pi_{0}^{2}-\pi^{T} \bar{x}(B)$ to be the amount by which the points in $\bar{x}(B)+L(B)$ violate the first and second terms in the disjunction respectively. Also, for $i \in B$, define

$$
\alpha_{i}\left(\pi, \pi_{0}^{1}, \pi_{0}^{2}, B\right):= \begin{cases}-\epsilon^{1}\left(\pi, \pi_{0}^{1}, B\right) /\left(\pi^{T} r^{i}(B)\right) & \text { if } \pi^{T} r^{i}(B)<0, \\ \epsilon^{2}\left(\pi, \pi_{0}^{2}, B\right) /\left(\pi^{T} r^{i}(B)\right) & \text { if } \pi^{T} r^{i}(B)>0 \\ +\infty & \text { otherwise. }\end{cases}
$$

The interpretation of the numbers $\alpha_{i}\left(\pi, \pi_{0}^{1}, \pi_{0}^{2}, B\right)$, for $i \in B$, is the following. For $\alpha \in \mathbb{R}_{+}$, let $x^{i}(\alpha, B):=\bar{x}(B)+\alpha r^{i}(B)$ denote the half-line starting at $\bar{x}(B)$ in the 
direction $r^{i}(B)$. The value $\alpha_{i}\left(\pi, \pi_{0}^{1}, \pi_{0}^{2}, B\right)$ is the smallest value of $\alpha \in \mathbb{R}_{+}$, if any, such that $x^{i}(\alpha, B)$ satisfies the disjunction $D\left(\pi, \pi_{0}^{1}, \pi_{0}^{2}\right)$ (see Figure 2(c) for an example). If the direction $r^{i}(B)$ is parallel to the hyperplanes $\pi^{T} x=\pi_{0}^{1}$ and $\pi^{T} x=\pi_{0}^{2}$, $\alpha_{i}\left(\pi, \pi_{0}^{1}, \pi_{0}^{2}, B\right)$ is defined to be $+\infty$. Given the numbers $\alpha_{i}\left(\pi, \pi_{0}^{1}, \pi_{0}^{2}, B\right)$ for $i \in B$, the intersection cut associated with $B$ and $D\left(\pi, \pi_{0}^{1}, \pi_{0}^{2}\right)$ is given by

$$
\sum_{i \in B}\left(b_{i}-a_{i .}^{T} x\right) / \alpha_{i}\left(\pi, \pi_{0}^{1}, \pi_{0}^{2}, B\right) \geq 1 .
$$

The validity of this inequality for $\operatorname{Conv}\left(P(B) \cap F_{D\left(\pi, \pi_{0}^{1}, \pi_{0}^{2}\right)}\right)$ was proven by Balas [2]. In fact, the intersection cut gives a complete description of the basic disjunctive hull associated with $B$ and $D\left(\pi, \pi_{0}^{1}, \pi_{0}^{2}\right)$.

Lemma 1. Let $B \in \mathcal{B}_{r}^{*}$ and $D\left(\pi, \pi_{0}^{1}, \pi_{0}^{2}\right)$ be a general split disjunction.

(i) If $\left.\pi^{T} x \notin\right] \pi_{0}^{1}, \pi_{0}^{2}\left[\right.$ for some $x \in \bar{x}(B)+L(B)$, then $\operatorname{Conv}\left(P(B) \cap F_{D\left(\pi, \pi_{0}^{1}, \pi_{0}^{2}\right)}\right)=$ $P(B)$.

(ii) If $\left.\pi^{T} x \in\right] \pi_{0}^{1}, \pi_{0}^{2}\left[\right.$ for all $x \in \bar{x}(B)+L(B)$, then $\operatorname{Conv}\left(P(B) \cap F_{D\left(\pi, \pi_{0}^{1}, \pi_{0}^{2}\right)}\right)=$ $\{x \in P(B):(3)\}$.

The proof of Lemma 1 will be given in Sect. 3 .

\subsection{Split disjunctions and polyhedra}

Having completely described the basic disjunctive hulls for general split disjunctions, a natural question is whether considering the intersection of all these sets is enough to describe the disjunctive hull associated with the polyhedron. The main result in this paper gives a positive answer to this question as follows.

Theorem 1. For every $\left(\pi, \pi_{0}^{1}, \pi_{0}^{2}\right)$ satisfying $\pi_{0}^{1}<\pi_{0}^{2}$,

$$
\operatorname{Conv}\left(P \cap F_{D\left(\pi, \pi_{0}^{1}, \pi_{0}^{2}\right)}\right)=\bigcap_{B \in \mathcal{B}_{r}^{*}} \operatorname{Conv}\left(P(B) \cap F_{D\left(\pi, \pi_{0}^{1}, \pi_{0}^{2}\right)}\right) .
$$

From a convex analysis perspective, this is an unusual result since it is typically not allowed to interchange the intersection operator with the convex hull operator. Note that $P=\cap_{B \in \mathcal{B}_{r}^{*}} P(B)$. Theorem 1 states that $\operatorname{Conv}\left(\cap_{B \in \mathcal{B}_{r}^{*}} P(B) \cap F_{D\left(\pi, \pi_{0}^{1}, \pi_{0}^{2}\right)}\right)=$ $\cap_{B \in \mathcal{B}_{r}^{*}} \operatorname{Conv}\left(P(B) \cap F_{D\left(\pi, \pi_{0}^{1}, \pi_{0}^{2}\right)}\right)$.

An immediate consequence of Theorem 1 is the following characterization of the split closure of a MILP, which implies that the split closure is completely described by intersection cuts.

\section{Corollary 1.}

$$
S C=\bigcap_{B \in \mathcal{B}_{r}^{*}} S C(B) .
$$

Another consequence is a new proof of the following fact.

Theorem 2. The split closure of MILP is a polyhedron. 


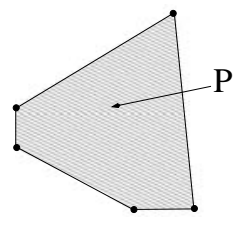

(a) A polytope

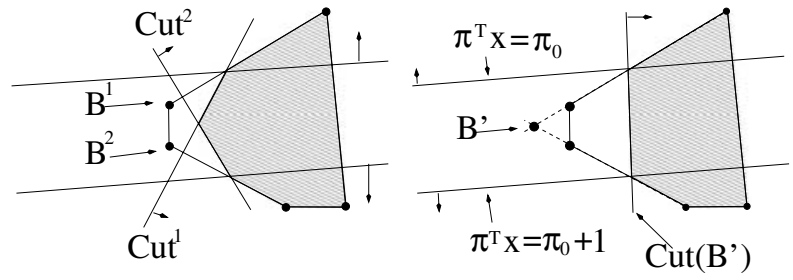

(b) Using only feasible bases

(c) Using an infeasible basis

Fig. 3. Infeasible bases are needed in Theorem 1

This was first proved by Cook, Kannan and Schrijver [6]. Our proof is based on showing that $\mathrm{SC}(B)$ is a polyhedron. Theorem 2 then follows from Corollary 1 .

When $r=n, \mathcal{B}_{r}^{*}$ is the set of bases of $A$. Every basis $B \in \mathcal{B}_{n}^{*}$ corresponds to a unique basic solution $\bar{x}(B)$, which may or may not be a feasible solution to LP. If $\bar{x}(B)$ is a feasible solution to LP, the basis $B$ is called a feasible basis, otherwise, it is called an infeasible basis. A natural question is whether infeasible bases are needed for Theorem 1 to be true. The example in Figure 3 demonstrates that infeasible bases are necessary. In Figure 3, the two intersection cuts derived from the two feasible bases $B^{1}$ and $B^{2}$ are both dominated by the cut derived from the infeasible basis $B^{\prime}$. In fact, the cut obtained from $B^{\prime}$ is the only necessary cut to describe $\operatorname{Conv}\left(P \cap F_{D\left(\pi, \pi_{0}\right)}\right)$.

\subsection{More general disjunctions}

Theorem 1 is proven for split disjunctions but the question of knowing if it generalizes to other disjunctions is still open. The example in Figure 4 demonstrates that Theorem 1 does not generalize from general split disjunctions to other types of disjunctions with two terms. In this example, $P$ has 3 constraints $a_{i}^{T} x \leq b_{i}, i=1,2,3$, and two bases $B^{1}$ and $B^{2} . D$ is a two-term disjunction involving the two hyperplanes $\left(\pi^{1}\right)^{T} x=\pi_{0}^{1}$ and $\left(\pi^{2}\right)^{T} x=\pi_{0}^{2}$. In Figure 4(b), the shaded area circumscribed by the cut and the two parallel lines form the constraints of $\operatorname{Conv}\left(P \cap F_{D}\right)$. In Figure 4(c), the shaded area is $\operatorname{Conv}\left(P\left(B^{1}\right) \cap F_{D}\right) \cap \operatorname{Conv}\left(P\left(B^{2}\right) \cap F_{D}\right)=\cap_{B \in \mathcal{B}_{r}^{*}} \operatorname{Conv}\left(P(B) \cap F_{D}\right)$. In this example, $\operatorname{Conv}\left(P \cap F_{D}\right) \neq \cap_{B \in \mathcal{B}_{r}^{*}} \operatorname{Conv}\left(P(B) \cap F_{D}\right)$.

Observe, however, that $\operatorname{Conv}\left(P \cap F_{D}\right)$ can be generated by considering $r$-subsets of the constraints $(r=2)$. In fact, in the example, only one 2 -subset needs to be considered (the set containing the parallel constraints of $P$ ). This does, in fact, hold for general two-term disjunctions $D$ of the form $D^{1} x \leq d^{1} \vee D^{2} x \leq d^{2}$, where $D^{1} \in \mathbb{R}^{m_{1} \times n}$, $D^{2} \in \mathbb{R}^{m_{2} \times n}, d^{1} \in \mathbb{R}^{m_{1}}$ and $d^{2} \in \mathbb{R}^{m_{2}}$.

Theorem 3. Let $D$ be a general two-term disjunction, and suppose $A$ is not of full row-rank, i.e., $|M|=m \geq r+1$. Define

(i) $\mathcal{C}_{1}^{*}:=\{S \subseteq M:|S|=r+1$ and $r(S)=r\}$.

(ii) $\mathcal{C}_{2}^{*}:=\{S \subseteq M:|S|=n$ and $(r(S)=n$ or $r(S)=n-1)\}$. 


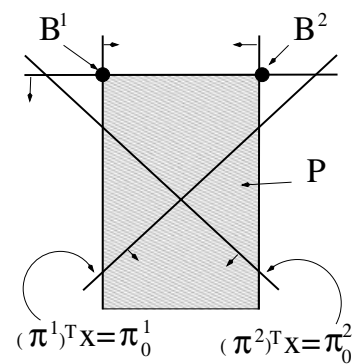

(a) A polyhedron and a disjunction

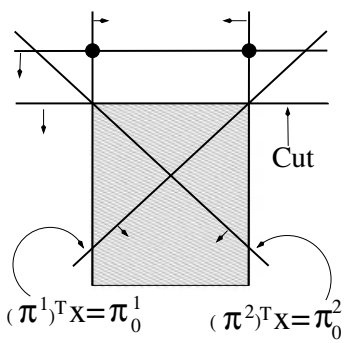

(b) The disjunctive hull

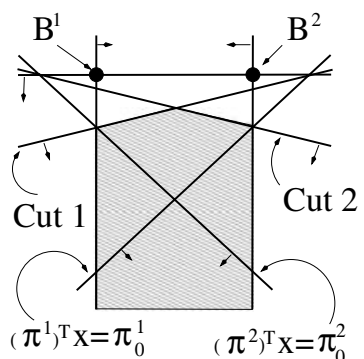

(c) Set obtained from bases

Fig. 4. Example of a general two-term disjunction

We have

$$
\operatorname{Conv}\left(P \cap F_{D}\right)=\bigcap_{T \in \mathcal{C}_{1}^{*}} \operatorname{Conv}\left(P(T) \cap F_{D}\right) .
$$

Furthermore, if $r=n$,

$$
\operatorname{Conv}\left(P \cap F_{D}\right)=\bigcap_{T \in \mathcal{C}_{2}^{*}} \operatorname{Conv}\left(P(T) \cap F_{D}\right) .
$$

A different counterexample for the generalization of Theorem 1 is given next. Theorem 1 demonstrates that the split closure of $P$ can be decomposed into split closures of the sets $P(B)$ for $B \in \mathcal{B}_{r}^{*}$. Is a similar decomposition result valid for the integer hull of $P$ ? In other words, do we have

$$
\operatorname{Conv}\left(P^{I}\right)=\bigcap_{B \in \mathcal{B}_{r}^{*}} \operatorname{Conv}\left(P^{I}(B)\right),
$$

where $P^{I}(B):=\left\{x \in P(B): x_{j}\right.$ integer, $\left.j \in N_{I}\right\}$ ? The following example shows that this is not true in 3 dimensions. Consider the polyhedron $P$ defined as the set of $x \in \mathbb{R}^{3}$ satisfying the following inequalities.

$$
\begin{aligned}
2 x_{1}-2 x_{2}+2 x_{3} & \leq 3, \\
-2 x_{1}+2 x_{2}+2 x_{3} & \leq 3, \\
2 x_{1}+2 x_{2}+2 x_{3} & \leq 5, \\
-2 x_{1}-2 x_{2}+2 x_{3} & \leq 1 .
\end{aligned}
$$

It can be verified that $\left(x_{1}, x_{2}, x_{3}\right)=\left(\frac{1}{2}, \frac{1}{2}, \frac{3}{2}\right)$ is the only point that satisfies all inequalities $(8)-(11)$ with equality. It can also be seen that $\left(x_{1}, x_{2}, x_{3}\right)=\left(\frac{1}{2}, \frac{1}{2}, \frac{1}{2}\right) \in$ $\operatorname{Conv}\left(P^{I}(B)\right)$ for all four bases $B \in \mathcal{B}_{3}^{*}$. However, the inequality $x_{3} \leq 0$ is valid for $\operatorname{Conv}\left(P^{I}\right)$. To see this, observe that the constraints (10) and (11), together with the 
integrality of $x_{3}$, imply $x_{3} \leq 1$. If $x_{3}=1$, by the integrality of $x_{1}$ and $x_{2}$, the constraints (8) and (9) imply $x_{1}-x_{2}=0$, while the constraints (10) and (11) imply $x_{1}+x_{2}=1$. Since no integral values of $x_{1}$ and $x_{2}$ satisfy $x_{1}-x_{2}=0$ and $x_{1}+x_{2}=1, P$ does not have an integral point with $x_{3}=1$.

\section{Conic polyhedra, cutting planes and split disjunctions}

In this section we focus on conic polyhedra. In Sect. 3.1 we consider conic polyhedra of the form

$$
\bar{C}:=\left\{x \in \mathbb{R}^{n}: x=\bar{x}+\sum_{j \in J} \bar{r}^{j} \mu_{j} \text { and } \mu_{j} \geq 0 \text { for } j \in J\right\},
$$

where $J$ is an index set, $\bar{x} \in \mathbb{R}^{n}, \bar{r}^{j} \in \mathbb{R}^{n}$ for $j \in J$ and the vectors $\left\{\bar{r}^{j}\right\}_{j \in J}$ are linearly independent. Conic polyhedra of the form $\bar{C}$ above are called simple conic polyhedra. We may have $|J|<n$. The focus in Sect. 3.1 is on inequalities that cut off $\bar{x}$, i.e., inequalities $\delta^{T} x \geq \delta_{0}$ satisfying $\delta^{T} \bar{x}<\delta_{0}$. Inequalities that cut off $\bar{x}$ are called apex-cuts for $\bar{C}$.

In Sect. 3.2 we consider simple conic polyhedra and general split disjunctions $\left(\pi, \pi_{0}^{1}, \pi_{0}^{2}\right)$. For split disjunctions that are violated by $\bar{x}$, we derive an intersection cut. Also, the intersection cut is used to describe the set $\operatorname{Conv}\left(\bar{C} \cap F_{D\left(\pi, \pi_{0}^{1}, \pi_{0}^{2}\right)}\right)$.

In Sect. 3.3 we prove that, given any subset $\Pi \subseteq \Pi^{n}\left(N_{I}\right)$ of split disjunctions, the set $\operatorname{SC}(\bar{C}, \Pi):=\cap_{\left(\pi, \pi_{0}\right) \in \Pi} \operatorname{Conv}\left(\bar{C} \cap F_{D\left(\pi, \pi_{0}\right)}\right)$ is a polyhedron. In words, $\operatorname{SC}(\bar{C}, \Pi)$ is defined to be the intersection of the disjunctive hulls defined by $\bar{C}$ and $D\left(\pi, \pi_{0}\right)$ over all the disjunctions $\left(\pi, \pi_{0}\right)$ in $\Pi$. Since the cardinality of $\Pi$ can be infinite, it is not trivial to see that $\operatorname{SC}(\bar{C}, \Pi)$ is a polyhedron. In particular, when $\Pi=\Pi^{n}\left(N_{I}\right)$, we know that the split closure of $\bar{C}$ is a polyhedron, as proved in [6].

In Sect. 3.4 we generalize the results of Sect. 3.3 on simple conic polyhedra $\bar{C}$ to conic polyhedra $P(B)$ defined in Sect. 1 and Sect. 2.1, where $B \in \mathcal{B}_{r}^{*}$. Given a general split disjunction $\left(\pi, \pi_{0}^{1}, \pi_{0}^{2}\right)$ that is violated by $\bar{x}(B)$, we characterize the disjunctive set obtained from $P(B)$ and $\left(\pi, \pi_{0}^{1}, \pi_{0}^{2}\right)$ as the set of points in $P(B)$ that satisfy the intersection cut. Furthermore, we show that the split closure of $P(B)$ is a polyhedron for all $B \in \mathcal{B}_{r}^{*}$.

\subsection{Simple conic polyhedra and apex-cuts}

We start by considering $\bar{C}$, the simple conic polyhedron defined in (12). The hyperplane associated with an intersection cut has intersection points with the extreme rays of $\bar{C}$. In fact, any apex-cut $\delta^{T} x \geq \delta_{0}$ for $\bar{C}$ has this characterization. For $j \in J$, define

$$
\alpha_{j}^{\prime}\left(\delta, \delta_{0}\right):= \begin{cases}\left(\delta_{0}-\delta^{T} \bar{x}\right) /\left(\delta^{T} \bar{r}^{j}\right) & \text { if } \delta^{T} \bar{r}^{j} \neq 0 \\ +\infty & \text { otherwise }\end{cases}
$$

Since $\delta^{T} x \geq \delta_{0}$ is an apex-cut for $\bar{C}, \delta_{0}>\delta^{T} \bar{x}$. As with the intersection cut, $\alpha_{j}^{\prime}\left(\delta, \delta_{0}\right)$ denotes the value of $\alpha$ for which the point $\bar{x}+\alpha \bar{r}^{j}$ is on the hyperplane $\delta^{T} x=\delta_{0}$. When 
there is no such point, $\alpha_{j}^{\prime}\left(\delta, \delta_{0}\right)=+\infty$. In contrast to the intersection cut, however, we might have $\alpha_{j}^{\prime}\left(\delta, \delta_{0}\right)<0$ for some $j \in J$. Let

$$
\bar{C}^{L}:=\left\{(x, \mu) \in \mathbb{R}^{n} \times \mathbb{R}^{|J|}: x=\bar{x}+\sum_{j \in J} \bar{r}^{j} \mu_{j} \text { and } \mu \geq 0_{|J|}\right\}
$$

be the set obtained from $\bar{C}$ by including the multipliers on the extreme rays of $\bar{C}$ in the description. The "L" in $\bar{C}^{L}$ is for "Lifted" since $\bar{C}^{L}$ is an image of $\bar{C}$ in a higher dimensional space (see Balas [3]). We have the following relationship between $\delta^{T} x \geq \delta_{0}$ and the scalars $\alpha_{j}^{\prime}\left(\delta, \delta_{0}\right)$ for $j \in J$.

Lemma 2. Let $\delta^{T} x \geq \delta_{0}$ be an arbitrary apex-cut for $\bar{C}$.

$$
\begin{aligned}
& \left\{x \in \bar{C}: \delta^{T} x \geq \delta_{0}\right\} \\
& \quad=\left\{x \in \bar{C}: \exists \mu \in \mathbb{R}^{|J|} \text { s.t. }(x, \mu) \in \bar{C}^{L} \text { and } \sum_{j \in J} \mu_{j} / \alpha_{j}^{\prime}\left(\delta, \delta_{0}\right) \geq 1\right\} .
\end{aligned}
$$

Proof. We have $x \in \bar{C}$ and $\delta^{T} x \geq \delta_{0} \Longleftrightarrow x=\bar{x}+\sum_{j \in J} \mu_{j} \bar{r}^{j}$, where $\mu \geq 0_{|J|}$, and $\delta^{T} x \geq \delta_{0} \Longleftrightarrow x=\bar{x}+\sum_{j \in J} \mu_{j} \bar{r}^{j}$, where $\mu \geq 0_{|J|}$, and $\sum_{j \in J} \mu_{j}\left(\delta^{T} \bar{r}^{j}\right) \geq$ $\left(\delta_{0}-\delta^{T} \bar{x}\right) \Longleftrightarrow(x, \mu) \in \bar{C}^{L}$ and $\sum_{j \in J} \mu_{j} / \alpha_{j}^{\prime}\left(\delta, \delta_{0}\right) \geq 1$.

Lemma 2 leads to the following notion of dominance between pairs of apex-cuts for $\bar{C}$. Given two apex-cuts $\left(\delta^{1}\right)^{T} x \geq \delta_{0}^{1}$ and $\left(\delta^{2}\right)^{T} x \geq \delta_{0}^{2}$ for $\bar{C}$, we say $\left(\delta^{1}\right)^{T} x \geq \delta_{0}^{1}$ dominates $\left(\delta^{2}\right)^{T} x \geq \delta_{0}^{2}$ on $\bar{C}$ if $1 / \alpha_{j}^{\prime}\left(\delta^{1}, \delta_{0}^{1}\right) \leq 1 / \alpha_{j}^{\prime}\left(\delta^{2}, \delta_{0}^{2}\right)$ for all $j \in J$. Furthermore, if $\left(\delta^{1}\right)^{T} x \geq \delta_{0}^{1}$ dominates $\left(\delta^{2}\right)^{T} x \geq \delta_{0}^{2}$ on $\bar{C}$ and $\left(\delta^{2}\right)^{T} x \geq \delta_{0}^{2}$ dominates $\left(\delta^{1}\right)^{T} x \geq \delta_{0}^{1}$ on $\bar{C}$, we say $\left(\delta^{1}\right)^{T} x \geq \delta_{0}^{1}$ and $\left(\delta^{2}\right)^{T} x \geq \delta_{0}^{2}$ are equivalent on $\bar{C}$. Two apex-cuts $\left(\delta^{1}\right)^{T} x \geq \delta_{0}^{1}$ and $\left(\delta^{2}\right)^{T} x \geq \delta_{0}^{2}$ are equivalent on $\bar{C}$ if and only if they intersect every ray at the same point, but the hyperplanes $\left(\delta^{1}\right)^{T} x=\delta_{0}^{1}$ and $\left(\delta^{2}\right)^{T} x=\delta_{0}^{2}$ are not necessarily identical as can be illustrated with a cone $\bar{C}$ with two rays in $\mathbb{R}^{3}$.

Observe that, if $\left(\delta^{1}\right)^{T} x \geq \delta_{0}^{1}$ dominates $\left(\delta^{2}\right)^{T} x \geq \delta_{0}^{2}$ on $\bar{C}$, then Lemma 2 implies $\left\{x \in \bar{C}:\left(\delta^{1}\right)^{T} x \geq \delta_{0}^{1}\right\} \subseteq\left\{x \in \bar{C}:\left(\delta^{2}\right)^{T} x \geq \delta_{0}^{2}\right\}$. Furthermore, if $\left(\delta^{1}\right)^{T} x \geq \delta_{0}^{1}$ is equivalent to $\left(\delta^{2}\right)^{T} \bar{x} \geq \delta_{0}^{2}$ on $\bar{C}$, then $\left\{x \in \bar{C}:\left(\delta^{1}\right)^{T} x \geq \delta_{0}^{1}\right\}=\left\{x \in \bar{C}:\left(\delta^{2}\right)^{T} x \geq \delta_{0}^{2}\right\}$.

We call an apex-cut $\delta^{T} x \geq \delta_{0}$ positive, if $\alpha_{j}^{\prime}\left(\delta, \delta_{0}\right)>0$ for all $j \in J$. In other words, $\delta^{T} x \geq \delta_{0}$ is positive if, for every $j \in J$ such that the hyperplane $\delta^{T} x=\delta_{0}$ intersects the line $\left\{\bar{x}+\alpha \bar{r}^{j}: \alpha \in \mathbb{R}\right\}$, the intersection point $\bar{x}+\alpha_{j}^{\prime}\left(\delta, \delta_{0}\right) \bar{r}^{j}$ is on the half-line contained in $\bar{C}$.

Now consider a polyhedron $\bar{P}$ obtained from $\bar{C}$ by adding the positive apex-cuts $\left(\delta^{1}\right)^{T} x \geq \delta_{0}^{1},\left(\delta^{2}\right)^{T} x \geq \delta_{0}^{2}, \ldots,\left(\delta^{p}\right)^{T} x \geq \delta_{0}^{p}$, i.e.

$$
\bar{P}:=\left\{x \in \bar{C}:\left(\delta^{k}\right)^{T} x \geq \delta_{0}^{k} \text { for } k=1,2, \ldots, p\right\} .
$$

Observe that $\bar{P} \neq \varnothing$ if and only if, for every $k \in\{1,2, \ldots, p\}$, there exists $j \in J$ such that $\alpha_{j}^{\prime}\left(\delta^{k}, \delta_{0}^{k}\right)<+\infty$. This is due to Lemma 2, i.e., by viewing the inequality $\left(\delta^{k}\right)^{T} x \geq \delta_{0}^{k}$ in the space of $(x, \mu)$ variables. By making the $\mu$ variables very large, a point in $\bar{C}$ can be constructed that satisfy the inequality $\left(\delta^{k}\right)^{T} x \geq \delta_{0}^{k}$.

Lemma 3. Assume $\bar{P} \neq \emptyset$. Let $\delta^{T} x \geq \delta_{0}$ be a positive apex-cut that is validfor $\bar{P}$. Then there exists an inequality $\left(\delta^{\prime}\right)^{T} x \geq \delta_{0}^{\prime}$, which can be obtained as a convex combination of the apex-cuts $\left(\delta^{k}\right)^{T} x \geq \delta_{0}^{k}, k=1,2, \ldots, p$, that dominates $\delta^{T} x \geq \delta_{0}$ on $\bar{C}$. 
Proof. Consider the LP $\min \left\{\delta^{T} x: x \in \bar{P}\right\}$. Since $\bar{P} \neq \varnothing$ and $\delta^{T} x \geq \delta_{0}$ is a valid inequality for $\bar{P}$, the LP is feasible and bounded. The dual of the LP gives vectors $\bar{u} \in \mathbb{R}^{n}$ and $\bar{v} \in \mathbb{R}^{p}$ such that

(a) $\bar{u}^{T} \bar{x}+\sum_{k=1}^{p} \delta_{0}^{k} \bar{v}_{k} \geq \delta_{0}$,

(b) $\bar{u}+\sum_{k=1}^{p} \bar{v}_{k} \delta^{k}=\delta$,

(c) $\bar{u}^{T} \bar{r}^{j} \geq 0$ for all $j \in J$ and

(d) $\bar{v} \geq 0$.

Define $\bar{\delta}:=\sum_{k=1}^{p} \bar{v}_{k} \delta^{k}$ and $\bar{\delta}_{0}:=\sum_{k=1}^{p} \delta_{0}^{k} \bar{v}_{k}$. The inequality $\bar{\delta}^{T} x \geq \bar{\delta}_{0}$ is a nonnegative combination of the inequalities $\left(\delta^{1}\right)^{T} x \geq \delta_{0}^{1},\left(\delta^{2}\right)^{T} x \geq \delta_{0}^{2}, \ldots,\left(\delta^{p}\right)^{T} x \geq \delta_{0}^{p}$, and is therefore valid for $\bar{P}$. We claim $\bar{\delta}^{T} x \geq \bar{\delta}_{0}$ dominates $\delta^{T} x \geq \delta_{0}$ on $\bar{C}$.

Let $j \in J$ be arbitrary. Observe that (a) and (b) give $\bar{u}^{T} \bar{x}+\bar{\delta}_{0} \geq \delta_{0}$ and $\bar{u}+\bar{\delta}=\delta$. This implies $\bar{u}^{T} \bar{r}^{j}+\bar{\delta}^{T} \bar{r}^{j}=\delta^{T} \bar{r}^{j}$. Since from (c) we have $\bar{u}^{T} \bar{r}^{j} \geq 0$, we also have $\bar{\delta}^{T} \bar{r}^{j} \leq \delta^{T} \bar{r}^{j}$. Furthermore, we have $\bar{u}^{T} \bar{x}+\bar{\delta}^{T} \bar{x}=\delta^{T} \bar{x}$ from multiplying $\bar{u}+\bar{\delta}=\delta$ by $\bar{x}$. Substituting the expression $\bar{u}^{T} \bar{x}=\delta^{T} \bar{x}-\bar{\delta}^{T} \bar{x}$ into the inequality $\bar{u}^{T} \bar{x}+\bar{\delta}_{0} \geq \delta_{0}$, we get $0<\delta_{0}-\delta^{T} \bar{x} \leq \bar{\delta}_{0}-\bar{\delta}^{T} \bar{x}$.

Observe that the assumption $\alpha^{\prime}\left(\delta, \delta_{0}\right) \geq 0$ implies $\delta^{T} \bar{r}^{j} \geq 0$. Now we have $0 \leq\left(\bar{\delta}^{T} \bar{r}^{j}\right) /\left(\bar{\delta}_{0}-\bar{\delta}^{T} \bar{x}\right) \leq\left(\delta^{T} \bar{r}^{j}\right) /\left(\bar{\delta}_{0}-\bar{\delta}^{T} \bar{x}\right) \leq\left(\delta^{T} \bar{r}^{j}\right) /\left(\delta_{0}-\delta^{T} \bar{x}\right)$. It follows that $1 / \alpha_{j}^{\prime}\left(\bar{\delta}, \bar{\delta}_{0}\right) \leq 1 / \alpha_{j}^{\prime}\left(\delta, \delta_{0}\right)$, so that $\bar{\delta}^{T} x \geq \bar{\delta}_{0}$ dominates $\delta^{T} x \geq \delta_{0}$ on $\bar{C}$.

Finally, the inequality $\bar{\delta}^{T} x \geq \bar{\delta}_{0}$ might not be a convex combination of the inequalities $\left(\delta^{1}\right)^{T} x \geq \delta_{0}^{1},\left(\delta^{2}\right)^{T} x \geq \delta_{0}^{2}, \ldots,\left(\delta^{p}\right)^{T} x \geq \delta_{0}^{p}$, i.e., we might have $\bar{v}^{S}:=$ $\sum_{k=1}^{p} \bar{v}_{k} \neq 1$. First we argue that $\bar{v} \neq 0_{p}$. If $\bar{v}=0_{p}$, then (a) and (b) imply $\delta^{T} \bar{x} \geq \delta_{0}$. Since $\delta^{T} x \geq \delta_{0}$ is an apex-cut for $\bar{C}$, this cannot be the case, so $\bar{v} \neq 0_{p}$. Now define $\delta^{\prime}:=\bar{\delta} / \bar{v}^{S}$ and $\delta_{0}^{\prime}:=\bar{\delta}_{0} / \bar{v}^{S}$. The inequality $\left(\delta^{\prime}\right)^{T} x \geq \delta_{0}^{\prime}$ is a convex combination of $\left(\delta^{1}\right)^{T} x \geq \delta_{0}^{1},\left(\delta^{2}\right)^{T} x \geq \delta_{0}^{2}, \ldots,\left(\delta^{p}\right)^{T} x \geq \delta_{0}^{p}$, and $\left(\delta^{\prime}\right)^{T} x \geq \delta_{0}^{\prime}$ is equivalent to $\bar{\delta}^{T} x \geq \bar{\delta}_{0}$ on $\bar{C}$, and therefore also dominates $\delta^{T} x \geq \delta_{0}$ on $\bar{C}$.

\subsection{Simple conic polyhedra and split disjunctions}

The intersection cut (3) given in Sect. 2 was derived using a description of a conic polyhedron as intersection of half-spaces $a_{i}^{T} x \leq b_{i}$. Since we do not have a description of $\bar{C}$ with half-spaces, we now give an alternative derivation using $\bar{x}$ and the extreme rays $\bar{r}^{j}$. Recall that the intersection cut goes through the intersection points of the disjunction with the extreme rays of the cone. define

Let $\left(\pi, \pi_{0}^{1}, \pi_{0}^{2}\right) \in \mathbb{R}^{n+2}$ satisfy $\pi_{0}^{1}<\pi_{0}^{2}$, and suppose $\left.\pi^{T} \bar{x} \in\right] \pi_{0}^{1}, \pi_{0}^{2}[$. Given $j \in J$,

$$
\bar{\alpha}_{j}\left(\pi, \pi_{0}^{1}, \pi_{0}^{2}\right):= \begin{cases}-\epsilon^{1}\left(\pi, \pi_{0}^{1}\right) /\left(\pi^{T} \bar{r}^{j}\right) & \text { if } \pi^{T} \bar{r}^{j}<0, \\ \epsilon^{2}\left(\pi, \pi_{0}^{2}\right) /\left(\pi^{T} \bar{r}^{j}\right) & \text { if } \pi^{T} \bar{r}^{j}>0, \\ +\infty & \text { otherwise }\end{cases}
$$

where $\epsilon^{1}\left(\pi, \pi_{0}^{1}\right):=\pi^{T} \bar{x}-\pi_{0}^{1}$ and $\epsilon^{2}\left(\pi, \pi_{0}^{2}\right):=\pi_{0}^{2}-\pi^{T} \bar{x}$. As mentioned in Sect. 2.1, $\bar{\alpha}_{j}\left(\pi, \pi_{0}^{1}, \pi_{0}^{2}\right)$, for $j \in J$, denotes the smallest value of $\alpha \geq 0$ such that $\bar{x}+\alpha \bar{r}^{j}$ satisfies the disjunction $D\left(\pi, \pi_{0}^{1}, \pi_{0}^{2}\right.$ ) (or $\bar{\alpha}_{j}\left(\pi, \pi_{0}^{1}, \pi_{0}^{2}\right)=+\infty$ if this does not happen). The intersection cut associated with $\bar{C}$ and $D\left(\pi, \pi_{0}^{1}, \pi_{0}^{2}\right)$ is given by

$$
\left(\delta\left(\pi, \pi_{0}^{1}, \pi_{0}^{2}\right)\right)^{T} x \geq \delta_{0}\left(\pi, \pi_{0}^{1}, \pi_{0}^{2}\right),
$$


where the vector $\delta\left(\pi, \pi_{0}^{1}, \pi_{0}^{2}\right)$ is one of possibly many solutions to the system

$$
\delta^{T} \bar{r}^{j}=1 / \bar{\alpha}_{j}\left(\pi, \pi_{0}^{1}, \pi_{0}^{2}\right), \forall j \in J
$$

and $\delta_{0}\left(\pi, \pi_{0}^{1}, \pi_{0}^{2}\right)$ is defined by $\delta_{0}\left(\pi, \pi_{0}^{1}, \pi_{0}^{2}\right):=\left(\delta\left(\pi, \pi_{0}^{1}, \pi_{0}^{2}\right)\right)^{T} \bar{x}+1$. Note that, since $\bar{r}^{j}, j \in J$, are linearly independent and the disjunction $D\left(\pi, \pi_{0}^{1}, \pi_{0}^{2}\right)$ gives an intersection cut, the equations (16) have a non-zero solution. The inequality $\left(\delta\left(\pi, \pi_{0}^{1}, \pi_{0}^{2}\right)\right)^{T} x \geq$ $\delta_{0}\left(\pi, \pi_{0}^{1}, \pi_{0}^{2}\right)$ has the property that, for all $j \in J$ satisfying $\pi^{T} \bar{r}^{j} \neq 0$, the point $\bar{x}+\bar{\alpha}_{j}\left(\pi, \pi_{0}^{1}, \pi_{0}^{2}\right) \bar{r}^{j}$ is on the hyperplane $\left(\delta\left(\pi, \pi_{0}^{1}, \pi_{0}^{2}\right)\right)^{T} x=\delta_{0}\left(\pi, \pi_{0}^{1}, \pi_{0}^{2}\right)$, i.e., the hyperplane of the intersection cut goes through all the intersection points of the disjunction and the cone. Furthermore, we have $\left(\delta\left(\pi, \pi_{0}^{1}, \pi_{0}^{2}\right)\right)^{T} \bar{x}<\delta_{0}\left(\pi, \pi_{0}^{1}, \pi_{0}^{2}\right)$, i.e., the hyperplane cuts off $\bar{x}$. Also observe that, for a general split disjunction $\left(\pi, \pi_{0}^{1}, \pi_{0}^{2}\right)$ and an extreme ray $j \in J$, we have the identity

$$
\bar{\alpha}_{j}\left(\pi, \pi_{0}^{1}, \pi_{0}^{2}\right)=\alpha_{j}^{\prime}\left(\delta\left(\pi, \pi_{0}^{1}, \pi_{0}^{2}\right), \delta_{0}\left(\pi, \pi_{0}^{1}, \pi_{0}^{2}\right)\right),
$$

where the scalars $\alpha_{j}^{\prime}\left(\delta\left(\pi, \pi_{0}^{1}, \pi_{0}^{2}\right), \delta_{0}\left(\pi, \pi_{0}^{1}, \pi_{0}^{2}\right)\right)$ are as defined in Sect. 3.1. Given $\bar{C}$ and $D\left(\pi, \pi_{0}^{1}, \pi_{0}^{2}\right)$, when $|J|<n$, the intersection cut is not unique, i.e., the equations (16) have more than just one solution. There can be many intersection cuts that intersect the half-lines of the cone at the same points.

The intersection cut is an apex-cut for $\bar{C}$. Because of Lemma 2, we have

$$
\{x \in \bar{C}:(15)\}=\left\{x \in \bar{C}: \exists \mu \in \mathbb{R}^{|J|} \text { s.t. }(x, \mu) \in \bar{C}^{L}, \sum_{j \in J} \mu_{j} / \bar{\alpha}_{j}\left(\pi, \pi_{0}^{1}, \pi_{0}^{2}\right) \geq 1\right\},
$$

where $\bar{C}^{L}$ was defined in Sect. 3.1. The next lemma characterizes $\operatorname{Conv}\left(\bar{C} \cap F_{D\left(\pi, \pi_{0}^{1}, \pi_{0}^{2}\right)}\right)$ for general split disjunctions $D\left(\pi, \pi_{0}^{1}, \pi_{0}^{2}\right)$.

Lemma 4. Let $\left(\pi, \pi_{0}^{1}, \pi_{0}^{2}\right) \in \mathbb{R}^{n+2}$ be an arbitrary general split disjunction, where $\pi_{0}^{1}<\pi_{0}^{2}$.

(i) If $\left.\pi^{T} \bar{x} \notin\right] \pi_{0}^{1}, \pi_{0}^{2}\left[\right.$, then $\operatorname{Conv}\left(\bar{C} \cap F_{D\left(\pi, \pi_{0}^{1}, \pi_{0}^{2}\right)}\right)=\bar{C}$,

(ii) If $\left.\pi^{T} \bar{x} \in\right] \pi_{0}^{1}, \pi_{0}^{2}\left[\right.$, then $\operatorname{Conv}\left(\bar{C} \cap F_{D\left(\pi, \pi_{0}^{1}, \pi_{0}^{2}\right)}\right)=\{x \in \bar{C}:(15)\}$.

Proof. (i) Assume $\bar{x} \in F_{D\left(\pi, \pi_{0}^{1}, \pi_{0}^{2}\right)}$. Wlog suppose $\pi^{T} \bar{x} \leq \pi_{0}^{1}$. Clearly $\operatorname{Conv}(\bar{C} \cap$ $F_{D\left(\pi, \pi_{0}^{1}, \pi_{0}^{2}\right)} \subseteq \bar{C}$ since $\bar{C}$ is convex, so we only need to show the other direction. Let $y \in \bar{C}$ be arbitrary. We may write $y=\bar{x}+\sum_{j \in J} s_{j} \bar{r}^{j}$, where $s_{j} \geq 0$ for $j \in J$. If $y \in F_{D\left(\pi, \pi_{0}^{1}, \pi_{0}^{2}\right)}$, then $y \in \bar{C} \cap F_{D\left(\pi, \pi_{0}^{1}, \pi_{0}^{2}\right)}$ and we are done. If not $\left.\pi^{T} y \in\right] \pi_{0}^{1}, \pi_{0}^{2}[$. Define $J^{+}:=\left\{j \in J: \pi^{T} \bar{r}^{j}>0\right.$ and $\left.s_{j}>0\right\}$. Since $\left.\pi^{T} y \in\right] \pi_{0}^{1}, \pi_{0}^{2}\left[\right.$ and $\pi^{T} \bar{x} \leq \pi_{0}^{1}$, we must have $J^{+} \neq \emptyset$. Let $z^{+}:=\sum_{j \in J^{+}} s_{j} \bar{r}^{j} \neq 0_{n}$, and let $z^{-}:=\sum_{j \in J \backslash J^{+}} s_{j} \bar{r}^{j}$. So $\pi^{T} z^{+}=\sum_{j \in J^{+}} S_{j}\left(\pi^{T} \bar{r}^{j}\right)>0$. Choose $\left.\left.\lambda \in\right] 0,1\right]$ to satisfy $\pi^{T}\left(\bar{x}+z^{-}+\frac{1}{\lambda} z^{+}\right) \geq \pi_{0}^{2}$. Define $y^{1}:=\bar{x}+z^{-}+\frac{1}{\lambda} z^{+} \in \bar{C}$ and $y^{2}:=\bar{x}+z^{-} \in \bar{C}$. Then $y=\bar{x}+z^{-}+z^{+}=\lambda y^{1}+$ $(1-\lambda) y^{2}$. By the choice of $\lambda, y^{1}$ satisfies the disjunction $D\left(\pi, \pi_{0}^{1}, \pi_{0}^{2}\right)$. Furthermore, since $\pi^{T}\left(\bar{x}+z^{-}\right) \leq \pi_{0}^{1}, y^{2}$ satisfies the disjunction $D\left(\pi, \pi_{0}^{1}, \pi_{0}^{2}\right)$. Therefore we have $\left.y^{1} \in \bar{C} \cap F_{D\left(\pi, \pi_{0}^{1}, \pi_{0}^{2}\right)}\right)$ and $\left.y^{2} \in \bar{C} \cap F_{D\left(\pi, \pi_{0}^{1}, \pi_{0}^{2}\right)}\right)$, so that $y \in \operatorname{Conv}\left(\bar{C} \cap F_{D\left(\pi, \pi_{0}^{1}, \pi_{0}^{2}\right)}\right)$. 
(ii) The validity of the intersection cut was proved by Balas [2]. We therefore know that (15) is valid for $\operatorname{Conv}\left(\bar{C} \cap F_{D\left(\pi, \pi_{0}^{1}, \pi_{0}^{2}\right)}\right)$. Since $\bar{C}$ is convex, we have the inclusion $\operatorname{Conv}\left(\bar{C} \cap F_{D\left(\pi, \pi_{0}^{1}, \pi_{0}^{2}\right)}\right) \subseteq\{x \in \bar{C}:(15)\}$. Let $y \in\{x \in \bar{C}:$ (15) $\}$ be arbitrary. If $y \in F_{D\left(\pi, \pi_{0}^{1}, \pi_{0}^{2}\right)}$, we are done, so suppose $\left.\pi^{T} y \in\right] \pi_{0}^{1}, \pi_{0}^{2}[$. We may write $y=\bar{x}+\sum_{j \in J} s_{j} \bar{r}^{j}$, where $s_{j} \geq 0$ for $j \in J$. Observe that Lemma 2 applied on the intersection cut (15) implies that $\sum_{j \in J} s_{j} / \bar{\alpha}_{j}\left(\pi, \pi_{0}^{1}, \pi_{0}^{2}\right) \geq 1$.

First assume $\sum_{j \in J} s_{j} / \bar{\alpha}_{j}\left(\pi, \pi_{0}^{1}, \pi_{0}^{2}\right)=1$. Define the set $J^{\neq 0}:=\left\{j \in J: \pi^{T} \bar{r}^{j} \neq\right.$ $0\}$ and define the non-negative scalars $\lambda_{j}:=s_{j} / \bar{\alpha}_{j}\left(\pi, \pi_{0}^{1}, \pi_{0}^{2}\right)$ for $j \in J \neq 0$. Clearly $\sum_{j \in J \neq 0} \lambda_{j}=1$. Also, let $\bar{z}:=\sum_{j \in J \backslash J \neq 0} s_{j} \bar{r}^{j}$. We have $y=\bar{x}+\sum_{j \in J} s_{j} \bar{r}^{j}=$ $\bar{x}+\bar{z}+\sum_{j \in J \neq 0} s_{j} \bar{r}^{j}$. This can be re-written as $y=\sum_{j \in J \neq 0} \lambda_{j}\left(\bar{x}+\bar{z}+\bar{\alpha}_{j}\left(\pi, \pi_{0}^{1}, \pi_{0}^{2}\right) \bar{r}^{j}\right)$. Now $y=\sum_{j \in J \neq 0} \lambda_{j} \bar{x}^{j}\left(\pi, \pi_{0}^{1}, \pi_{0}^{2}\right)$ is a convex combination of the vectors $\left\{\bar{x}^{j}\left(\pi, \pi_{0}^{1}, \pi_{0}^{2}\right)\right\}_{j \in J}$, which are defined as $\bar{x}^{j}\left(\pi, \pi_{0}^{1}, \pi_{0}^{2}\right):=\bar{x}+\bar{z}+\bar{\alpha}_{j}\left(\pi, \pi_{0}^{1}, \pi_{0}^{2}\right) \bar{r}^{j}$ for $j \in J$. Since all the vectors $\left\{\bar{x}^{j}\left(\pi, \pi_{0}^{1}, \pi_{0}^{2}\right)\right\}_{j \in J}$ satisfy the disjunction $D\left(\pi, \pi_{0}^{1}, \pi_{0}^{2}\right)$, this shows $y \in \operatorname{Conv}\left(\bar{C} \cap F_{D\left(\pi, \pi_{0}^{1}, \pi_{0}^{2}\right)}\right)$.

Now suppose $\sum_{j \in J} s_{j} / \alpha_{j}\left(\pi, \pi_{0}^{1}, \pi_{0}^{2}\right)>1$. Let $z$ be the point on the line between $y$ and $\bar{x}$ that satisfies (15) with equality. We have $y=\bar{x}+\mu(z-\bar{x})$ for some $\mu>1$. Also, we know $z$ can be expressed as $z=\lambda^{1} z^{1}+\lambda^{2} z^{2}$, where $z^{1}, z^{2} \in \bar{C} \cap F_{D\left(\pi, \pi_{0}^{1}, \pi_{0}^{2}\right)}$, $\lambda^{1}, \lambda^{2} \geq 0$ and $\lambda^{1}+\lambda^{2}=1$. Then $y=\lambda^{1}\left(\bar{x}+\mu\left(z^{1}-\bar{x}\right)\right)+\lambda^{2}\left(\bar{x}+\mu\left(z^{2}-\bar{x}\right)\right) \in$ $\operatorname{Conv}\left(\overline{\bar{C}} \cap F_{D\left(\pi, \pi_{0}^{1}, \pi_{0}^{2}\right)}\right)$ since $\bar{x}+\mu\left(z^{i}-\bar{x}\right) \in \bar{C} \cap F_{D\left(\pi, \pi_{0}^{1}, \pi_{0}^{2}\right)}$ for $i=1,2$.

\subsection{Polyhedrality of the split closure of a simple conic polyhedron}

We now prove that the split closure of a simple conic polyhedron is a polyhedron. Given a set $\Pi \subseteq \Pi^{n}\left(N_{I}\right)$ of split disjunctions, define $\operatorname{SC}(\bar{C}, \Pi):=\cap_{\left(\pi, \pi_{0}\right) \in \Pi} \operatorname{Conv}(\bar{C} \cap$ $\left.F_{D\left(\pi, \pi_{0}\right)}\right)$. We will prove that $\operatorname{SC}(\bar{C}, \Pi)$ is a polyhedron. Wlog assume $\Pi$ satisfies $\pi^{T} \bar{x} \in$ ]$\pi_{0}^{1}, \pi_{0}^{2}\left[\right.$ for all $\left(\pi, \pi_{0}\right) \in \Pi$ (Lemma 4$)$.

Observe that every intersection cut is characterized by its intersection points with the extreme rays of $\bar{C}$. Furthermore, any two intersection cuts that intersect every ray at the same point are equivalent. Therefore, we only need to show that the number of possible intersection points with each extreme ray is finite.

Throughout this section we assume $\bar{x} \in \mathbb{Q}^{n}$ and $\bar{r}^{j} \in \mathbb{Z}^{n}$ for all $j \in J$. This is equivalent to assuming that the simple conic polyhedron $\bar{C}$ is rational. Let $\left(\pi, \pi_{0}\right) \in \Pi$ be an arbitrary split disjunction. For simplicity, let $\delta\left(\pi, \pi_{0}\right)$ and $\delta_{0}\left(\pi, \pi_{0}\right)$ describe the intersection cut $\left(\delta\left(\pi, \pi_{0}\right)\right)^{T} x \geq \delta_{0}\left(\pi, \pi_{0}\right)$ associated with $\bar{C}$ and $D\left(\pi, \pi_{0}\right)$, and let $\bar{\alpha}_{j}\left(\pi, \pi_{0}\right), j \in J$, be the coefficients (14) in the intersection cut. Furthermore, let $\epsilon^{1}\left(\pi, \pi_{0}\right)$ and $\epsilon^{2}\left(\pi, \pi_{0}\right)$ denote the amounts by which $\bar{x}$ violates the first and second terms of the disjunction $D\left(\pi, \pi_{0}\right)$, respectively.

The following is a key lemma, which gives an expression for $\bar{\alpha}_{j}\left(\pi, \pi_{0}\right)$ for a disjunction $D\left(\pi, \pi_{0}\right)$ and an extreme ray $j \in J$.

Lemma 5. Let $\left(\pi, \pi_{0}\right) \in \Pi$ and $j \in J$ satisfy $\bar{\alpha}_{j}\left(\pi, \pi_{0}\right)<+\infty$. Then there exist integers $p_{j}\left(\pi, \pi_{0}\right), w_{j}\left(\pi, \pi_{0}\right)$ and $g$ such that 
(i) $\bar{\alpha}_{j}\left(\pi, \pi_{0}\right)<1$.

(ii) $\bar{\alpha}_{j}\left(\pi, \pi_{0}\right)=\frac{p_{j}\left(\pi, \pi_{0}\right)}{g w_{j}\left(\pi, \pi_{0}\right)}$, where $0<p_{j}\left(\pi, \pi_{0}\right)<g$ and $w_{j}\left(\pi, \pi_{0}\right)>0$.

Proof. (i) Since $\bar{r}^{j}$ and $\pi$ are integers and $\pi^{T} \bar{r}^{j} \neq 0$, we must have $\left|\pi^{T} \bar{r}^{j}\right| \geq 1$. Because every split disjunction is such that $\pi_{0}^{2}-\pi_{0}^{1}=1$, we have $\left.\epsilon^{1}\left(\pi, \pi_{0}\right), \epsilon^{2}\left(\pi, \pi_{0}\right) \in\right] 0,1[$. Therefore, (14) implies $\bar{\alpha}_{j}\left(\pi, \pi_{0}\right)<1$.

(ii) Since $\bar{x}$ is rational, we may write $\bar{x}=\left(\frac{p_{1}}{q_{1}}, \frac{p_{2}}{q_{2}}, \ldots, \frac{p_{n}}{q_{n}}\right)$, where $p_{i} \in \mathbb{Z}$ and $q_{i} \in \mathbb{N}$ for $i=1,2, \ldots, n$. Define $d_{k}:=\Pi_{i=1, i \neq k}^{n} q_{i}$ for $k=1,2, \ldots, n$, and let $g:=\Pi_{i=1}^{n} q_{i}>0$. If $\pi^{T} \bar{r}^{j}<0$, let $p_{j}\left(\pi, \pi_{0}\right):=\sum_{k=1}^{n} d_{k} p_{k} \pi_{k}-g \pi_{0}$ and $w_{j}\left(\pi, \pi_{0}\right):=$ $-\pi^{T} \bar{r}^{j}>0$. Finally, if $\pi^{T} \bar{r}^{j}>0$, let $p_{j}\left(\pi, \pi_{0}\right):=g+g \pi_{0}-\sum_{k=1}^{n} d_{k} p_{k} \pi_{k}$ and $w_{j}\left(\pi, \pi_{0}\right):=\pi^{T} \bar{r}^{j}>0$. With these choices, (14) implies $\bar{\alpha}_{j}\left(\pi, \pi_{0}\right)=\frac{p_{j}\left(\pi, \pi_{0}\right)}{g w_{j}\left(\pi, \pi_{0}\right)}$. We also have $0<p_{j}\left(\pi, \pi_{0}\right)<g$ because $\left.\epsilon^{1}\left(\pi, \pi_{0}\right), \epsilon^{2}\left(\pi, \pi_{0}\right) \in\right] 0,1[$.

In Lemma 5, we observe that the integer $g$ is independent of the disjunction $D\left(\pi, \pi_{0}\right)$ and the extreme ray $j \in J$. The following lemma, which will be used to prove that $\operatorname{SC}(\bar{C}, \Pi)$ is a polyhedron, bounds the number of intersection points the split disjunctions in $\Pi$ can have with half-lines of the form $\left\{\bar{x}+\alpha r^{j}: \alpha \geq \alpha^{*}\right\}$, where $j \in J$ and $\alpha^{*}>0$ is arbitrary.

Lemma 6. For any $j \in J$ and $\alpha^{*}>0$, the split disjunctions $\left(\pi, \pi_{0}\right) \in \Pi$ only have a finite number of intersection points $\bar{x}+\bar{\alpha}_{j}\left(\pi, \pi_{0}\right) r^{j}$ with the half-line $\left\{\bar{x}+\alpha r^{j}: \alpha \geq \alpha^{*}\right\}$.

Proof. We only have to prove that the number of possible values for $\bar{\alpha}_{j}\left(\pi, \pi_{0}\right)$ satisfying $\bar{\alpha}_{j}\left(\pi, \pi_{0}\right) \geq \alpha^{*}$ is finite. Therefore let $j \in J$ and $\left(\pi, \pi_{0}\right) \in \Pi$ be arbitrary. Wlog assume $\bar{\alpha}_{j}\left(\pi, \pi_{0}\right)<\infty$, and that $\alpha^{*}=\frac{p^{*}}{g w^{*}}$, where $p^{*}, w^{*} \in \mathbb{Z}, 0<p^{*}<g$ and $w^{*}>0$.

From Lemma 5 we have that $0<\alpha^{*} \leq \bar{\alpha}_{j}\left(\pi, \pi_{0}\right)=\frac{p_{j}\left(\pi, \pi_{0}\right)}{g w_{j}\left(\pi, \pi_{0}\right)}<1$. Now, there is only a finite number of possible values for $p_{j}\left(\pi, \pi_{0}\right)$, namely the values $1,2, \ldots,(g-1)$. Also, for every $p \in\{1,2, \ldots,(g-1)\}$, a corresponding value $w$ must satisfy $1>\frac{p}{g w} \geq$ $\frac{p^{*}}{g w^{*}}$, which means $\frac{p}{g}<w \leq \frac{p w^{*}}{p^{*}}$.

We now prove that $\operatorname{SC}(\bar{C}, \Pi)$ is a polyhedron. The proof is by induction on $|J|$. First consider the case of $|J|=1$ (basic step).

Lemma 7. Suppose $|J|=1$. Then there exists $\left(\pi^{*}, \pi_{0}^{*}\right) \in \Pi$ such that $\operatorname{SC}(\bar{C}, \Pi)=$ $\left\{x \in \bar{C}:\left(\delta\left(\pi^{*}, \pi_{0}^{*}\right)\right)^{T} x \geq \delta_{0}\left(\pi^{*}, \pi_{0}^{*}\right)\right\}$.

Proof. We may assume that $J=\{1\}$. We have $\bar{C}=\left\{x \in \mathbb{R}^{n}: x=\bar{x}+\alpha \bar{r}^{1}, \alpha \geq 0\right\}$. First suppose there exists $\left(\pi^{*}, \pi_{0}^{*}\right) \in \Pi$ such that $\left(\pi^{*}\right)^{T} \bar{r}^{1}=0$. This implies that the intersection cut associated with $\left(\pi^{*}, \pi_{0}^{*}\right)$ cuts off the entire ray $\bar{C}$.

Now suppose $\pi^{T} \bar{r}^{1} \neq 0$ for all $\left(\pi, \pi_{0}\right) \in \Pi$. Define $\alpha_{1}^{*}:=\sup \left\{\bar{\alpha}_{1}\left(\pi, \pi_{0}\right):\left(\pi, \pi_{0}\right) \in\right.$ $\Pi\}$. Then we have $\operatorname{SC}(\bar{C}, \Pi)=\left\{x \in \mathbb{R}^{n}: x=\bar{x}+\alpha \bar{r}^{1}, \alpha \geq \alpha_{1}^{*}\right\}$. Hence the only question is whether the supremum is achieved by some $\left(\pi, \pi_{0}\right) \in \Pi$.

Let $\left(\pi^{\prime}, \pi_{0}^{\prime}\right) \in \Pi$ be arbitrary, and let $p^{\prime}:=p\left(\pi^{\prime}, \pi_{0}^{\prime}\right)$ and $w^{\prime}=w\left(\pi^{\prime}, \pi_{0}^{\prime}\right)$ be as in Lemma 5. For every integer $p$ satisfying $0<p<g$ there are only a finite number of integers $w>0$ for which $\frac{p}{w g}>\frac{p^{\prime}}{w^{\prime} g}$, namely integers $w>0$ satisfying $w<\frac{p w^{\prime}}{p^{\prime}}$. Since 
there are also only a finite number of integers $p$ satisfying $0<p<g$, there are only a finite number of possible integers $p$ and $w$ such that $0<p<g$ and $w>0$ for which $\frac{p}{w g}>\frac{p^{\prime}}{w^{\prime} g}$. Hence the supremum is achieved.

We now discuss the induction hypothesis. Given $j \in J$, let

$$
\bar{C}^{j}:=\bar{x}+\text { Cone }\left(\left\{\bar{r}^{k}\right\}_{k \in J \backslash\{j\}}\right),
$$

and let $\operatorname{SC}\left(\bar{C}^{j}, \Pi\right):=\bigcap_{\left(\pi, \pi_{0}\right) \in \Pi} \operatorname{Conv}\left(\bar{C}^{j} \cap F_{D\left(\pi, \pi_{0}\right)}\right)$. The induction hypothesis is that there exists a finite set $\Pi^{j} \subseteq \Pi$ of split disjunctions such that

$$
\operatorname{SC}\left(\bar{C}^{j}, \Pi\right)=\left\{x \in \bar{C}^{j}:\left(\delta\left(\pi, \pi_{0}\right)\right)^{T} x \geq \delta_{0}\left(\pi, \pi_{0}\right) \text { for all }\left(\pi, \pi_{0}\right) \in \Pi^{j}\right\},
$$

i.e., $\operatorname{SC}\left(\bar{C}^{j}, \Pi\right)$ is the set of points in $\bar{C}^{j}$ satisfying the intersection cuts associated with $\bar{C}^{j}$ and split disjunctions $\left(\pi, \pi_{0}\right)$ in $\Pi^{j}$. Furthermore, for $\left(\pi, \pi_{0}\right) \in \Pi^{j}, \delta\left(\pi, \pi_{0}\right)$ is a solution to the system

$$
\delta^{T} \bar{r}^{k}=1 / \bar{\alpha}_{k}\left(\pi, \pi_{0}\right), \forall k \in J \backslash\{j\}
$$

and $\delta_{0}\left(\pi, \pi_{0}\right):=\left(\delta\left(\pi, \pi_{0}\right)\right)^{T} \bar{x}+1$. Let $\left(\pi, \pi_{0}\right) \in \Pi^{j}$ be arbitrary. Since the equations in (18) are a subset of those in (16), we can choose $\delta\left(\pi, \pi_{0}\right)$ to be a solution to (16) such that it automatically satisfies (18). In other words, we can choose an intersection cut associated with $\bar{C}^{j}$ and $\left(\pi, \pi_{0}\right)$ which is also an intersection cut associated with $\bar{C}$ and $\left(\pi, \pi_{0}\right)$. We now prove that $\operatorname{SC}(\bar{C}, \Pi)$ is a polyhedron.

Lemma 8. $S C(\bar{C}, \Pi)$ is a polyhedron.

Proof. For each $j \in J$, define $\alpha_{j}^{*}:=\min \left\{\bar{\alpha}_{j}\left(\pi, \pi_{0}\right):\left(\pi, \pi_{0}\right) \in \Pi^{j}\right\}>0$. The scalar $\alpha_{j}^{*}$ is such that $\bar{x}+\alpha_{j}^{*} \bar{r}^{j}$ is the intersection point on the half-line $\bar{x}+\alpha \bar{r}^{j}, \alpha \geq 0$, which is closest to $\bar{x}$ over all disjunctions $\left(\pi, \pi_{0}\right)$ in $\Pi^{j}$.

Let $\bar{\Pi}:=\cup_{j \in J} \Pi^{j}$, and let $\operatorname{SC}(\bar{C}, \bar{\Pi}):=\bigcap_{\left(\pi, \pi_{0}\right) \in \bar{\Pi}} \operatorname{Conv}\left(\bar{C} \cap F_{D\left(\pi, \pi_{0}\right)}\right)$ denote the approximation to $\operatorname{SC}(\bar{C}, \Pi)$ obtained by considering the finite set of split disjunctions in $\bar{\Pi}$.

Consider a split disjunction $\left(\pi^{\prime}, \pi_{0}^{\prime}\right) \in \Pi \backslash \bar{\Pi}$. We will show that, if $\bar{\alpha}_{j}\left(\pi^{\prime}, \pi_{0}^{\prime}\right)<$ $\alpha_{j}^{*}$ for some $j \in J$, then the intersection cut $\left(\delta\left(\pi^{\prime}, \pi_{0}^{\prime}\right)\right)^{T} x \geq \delta_{0}\left(\pi^{\prime}, \pi_{0}^{\prime}\right)$ is valid for $\operatorname{SC}(\bar{C}, \bar{\Pi})$. This will mean that it is only necessary to consider split disjunctions in $\Pi \backslash \bar{\Pi}$ satisfying $\bar{\alpha}_{j}\left(\pi, \pi_{0}\right) \geq \alpha_{j}^{*}$ for all $j \in J$. Since, for every extreme ray $j \in J$ and split disjunction $\left(\pi, \pi_{0}\right) \in \Pi \backslash \bar{\Pi}$, there is only a finite number of possible values for $\bar{\alpha}_{j}\left(\pi, \pi_{0}\right)$ satisfying $\bar{\alpha}_{j}\left(\pi, \pi_{0}\right) \geq \alpha_{j}^{*}$ (Lemma 6), and since intersection cuts that intersect every extreme ray of $\bar{C}$ at the same points are equivalent, this shows that it is sufficient to consider a finite number of disjunctions in $\Pi \backslash \bar{\Pi}$ to get $\operatorname{SC}(\bar{C}, \Pi)$ from $\operatorname{SC}(\bar{C}, \bar{\Pi})$. It then follows that $\operatorname{SC}(\bar{C}, \Pi)$ is a polyhedron.

Therefore assume $\left(\pi^{\prime}, \pi_{0}^{\prime}\right) \in \Pi \backslash \bar{\Pi}$ and $j \in J$ satisfies $\bar{\alpha}_{j}\left(\pi^{\prime}, \pi_{0}^{\prime}\right)<\alpha_{j}^{*}$. Because $\left(\delta\left(\pi^{\prime}, \pi_{0}^{\prime}\right)\right)^{T} x \geq \delta_{0}\left(\pi^{\prime}, \pi_{0}^{\prime}\right)$ is an intersection cut associated with $\bar{C}$ and $\left(\pi^{\prime}, \pi_{0}^{\prime}\right)$, it is a positive apex-cut for $\bar{C}$ and $\bar{C}^{j}$. Since $\bar{C}^{j} \subseteq \bar{C}$, we have $\operatorname{SC}\left(\bar{C}^{j}, \Pi\right) \subseteq \operatorname{SC}(\bar{C}, \Pi)$, which implies $\left(\delta\left(\pi^{\prime}, \pi_{0}^{\prime}\right)\right)^{T} x \geq \delta_{0}\left(\pi^{\prime}, \pi_{0}^{\prime}\right)$ is valid for $\operatorname{SC}\left(\bar{C}^{j}, \Pi\right)$. By Lemma 3 there exists an inequality $\left(\delta^{\prime}\right)^{T} x \geq \delta_{0}^{\prime}$ that dominates $\left(\delta\left(\pi^{\prime}, \pi_{0}^{\prime}\right)\right)^{T} x \geq \delta_{0}\left(\pi^{\prime}, \pi_{0}^{\prime}\right)$ on $\bar{C}^{j}$ and 
can be expressed as a convex combination of the inequalities $\left(\delta\left(\pi, \pi_{0}\right)\right)^{T} x \geq \delta_{0}\left(\pi, \pi_{0}\right)$ for $\left(\pi, \pi_{0}\right) \in \Pi^{j}$. Assume $\delta^{\prime}=\sum_{\left(\pi, \pi_{0}\right) \in \Pi^{j}} \delta\left(\pi, \pi_{0}\right) \lambda_{\left(\pi, \pi_{0}\right)}$ and $\delta_{0}^{\prime}=\sum_{\left(\pi, \pi_{0}\right) \in \Pi^{j}}$ $\delta_{0}\left(\pi, \pi_{0}\right) \lambda_{\left(\pi, \pi_{0}\right)}$, where $\sum_{\left(\pi, \pi_{0}\right) \in \Pi^{j}} \lambda_{\left(\pi, \pi_{0}\right)}=1$ and $\lambda_{\left(\pi, \pi_{0}\right)} \geq 0$ for all $\left(\pi, \pi_{0}\right) \in \Pi^{j}$.

To finish the proof, it suffices to show that $\left(\delta^{\prime}\right)^{T} x \geq \delta_{0}^{\prime}$ also dominates $\left(\delta\left(\pi^{\prime}, \pi_{0}^{\prime}\right)\right)^{T} x \geq$ $\delta_{0}\left(\pi^{\prime}, \pi_{0}^{\prime}\right)$ on $\bar{C}$. Because $\left(\delta^{\prime}\right)^{T} x \geq \delta_{0}^{\prime}$ dominates $\left(\delta\left(\pi^{\prime}, \pi_{0}^{\prime}\right)\right)^{T} x \geq \delta_{0}\left(\pi^{\prime}, \pi_{0}^{\prime}\right)$ on $\bar{C}^{j}$, by the notation introduced in (13) we know $1 / \alpha_{i}^{\prime}\left(\delta^{\prime}, \delta_{0}^{\prime}\right) \leq 1 / \alpha_{i}^{\prime}\left(\delta\left(\pi^{\prime}, \pi_{0}^{\prime}\right), \delta_{0}\left(\pi^{\prime}, \pi_{0}^{\prime}\right)\right)$ for $i \in J \backslash\{j\}$. We only need to verify $1 / \alpha_{j}^{\prime}\left(\delta^{\prime}, \delta_{0}^{\prime}\right) \leq 1 / \alpha_{j}^{\prime}\left(\delta\left(\pi^{\prime}, \pi_{0}^{\prime}\right), \delta_{0}\left(\pi^{\prime}, \pi_{0}^{\prime}\right)\right)$.

By definition of $\delta_{0}\left(\pi, \pi_{0}\right), \delta_{0}\left(\pi, \pi_{0}\right)-\left(\delta\left(\pi, \pi_{0}\right)\right)^{T} \bar{x}=1$ for all $\left(\pi, \pi_{0}\right) \in \Pi^{j}$. By (16) and the choice of $\alpha_{j}^{*}$, we have $\delta_{0}\left(\pi, \pi_{0}\right)-\left(\delta\left(\pi, \pi_{0}\right)\right)^{T} \bar{x} \geq \alpha_{j}^{*}\left(\delta\left(\pi, \pi_{0}\right)\right)^{T} \bar{r}^{j}$. This implies $\sum_{\left(\pi, \pi_{0}\right) \in \Pi^{j}} \lambda_{\left(\pi, \pi_{0}\right)}\left(\delta_{0}\left(\pi, \pi_{0}\right)-\left(\delta\left(\pi, \pi_{0}\right)\right)^{T} \bar{x}\right) \geq \alpha_{j}^{*} \sum_{\left(\pi, \pi_{0}\right) \in \Pi^{j}} \lambda_{\left(\pi, \pi_{0}\right)}$ $\left(\delta\left(\pi, \pi_{0}\right)\right)^{T} \bar{r}^{j}$. Furthermore, we have the identities

(a) $\delta_{0}^{\prime}-\left(\delta^{\prime}\right)^{T} \bar{x}=\sum_{\left(\pi, \pi_{0}\right) \in \Pi^{j}} \lambda_{\left(\pi, \pi_{0}\right)}\left(\delta_{0}\left(\pi, \pi_{0}\right)-\left(\delta\left(\pi, \pi_{0}\right)\right)^{T} \bar{x}\right)$ and

(b) $\left(\delta^{\prime}\right)^{T} \bar{r}^{j}=\sum_{\left(\pi, \pi_{0}\right) \in \Pi^{j}} \lambda_{\left(\pi, \pi_{0}\right)}\left(\delta\left(\pi, \pi_{0}\right)\right)^{T} \bar{r}^{j}$.

From (a), (b) and (13) it follows that $\alpha_{j}^{*} \leq \alpha_{j}^{\prime}\left(\delta^{\prime}, \delta_{0}^{\prime}\right)$. The choice of $\alpha_{j}^{*}$ and (17) imply $\alpha_{j}^{\prime}\left(\delta\left(\pi^{\prime}, \pi_{0}^{\prime}\right), \delta_{0}\left(\pi^{\prime}, \pi_{0}^{\prime}\right)\right)<\alpha_{j}^{*}$, so that $\alpha_{j}^{\prime}\left(\delta\left(\pi^{\prime}, \pi_{0}^{\prime}\right), \delta_{0}\left(\pi^{\prime}, \pi_{0}^{\prime}\right)\right)<\alpha_{j}^{\prime}\left(\delta^{\prime}, \delta_{0}^{\prime}\right)$. Hence $1 / \alpha_{j}^{\prime}\left(\delta^{\prime}, \delta_{0}^{\prime}\right)<1 / \alpha_{j}^{\prime}\left(\delta\left(\pi^{\prime}, \pi_{0}^{\prime}\right), \delta_{0}\left(\pi^{\prime}, \pi_{0}^{\prime}\right)\right)$, which shows $\left(\delta^{\prime}\right)^{T} x \geq \delta_{0}^{\prime}$ dominates $\left(\delta\left(\pi^{\prime}, \pi_{0}^{\prime}\right)\right)^{T} x \geq \delta_{0}\left(\pi^{\prime}, \pi_{0}^{\prime}\right)$ on $\bar{C}$.

\subsection{Conic polyhedra and split disjunctions}

In this section we generalize the results of Sect. 3.2 and Sect. 3.3 to conic polyhedra of the form $P(B)$ of Sect. 2.1, where $B \in \mathcal{B}_{r}^{*}$. Throughout this section $B$ denotes an element of $\mathcal{B}_{r}^{*}$. We mention in Sect. 2.1 that $P(B)$ can be written as $P(B)=\bar{x}(B)+$ $L(B)+$ Cone $\left(\left\{r^{i}(B)\right\}_{i \in B}\right)$. Observe that the set $P^{\prime}(B):=\bar{x}(B)+$ Cone $\left(\left\{r^{i}(B)\right\}_{i \in B}\right)$ is a simple conic polyhedron.

We start by providing a link between the sets $\operatorname{Conv}\left(P(B) \cap F_{D\left(\pi, \pi_{0}^{1}, \pi_{0}^{2}\right)}\right)$ and $\operatorname{Conv}\left(P^{\prime}(B) \cap F_{D\left(\pi, \pi_{0}^{1}, \pi_{0}^{2}\right)}\right)$ for a general split disjunction $D\left(\pi, \pi_{0}^{1}, \pi_{0}^{2}\right)$ :

Lemma 9. Let $\left(\pi, \pi_{0}^{1}, \pi_{0}^{2}\right)$ be a general split disjunction, where $\pi_{0}^{1}<\pi_{0}^{2}$.

(i) If the linear function $\pi^{T} x$ is not constant on the affine space $\bar{x}(B)+L(B)$, then $\operatorname{Conv}\left(P(B) \cap F_{D\left(\pi, \pi_{0}^{1}, \pi_{0}^{2}\right)}\right)=P(B)$.

(ii) If the linear function $\pi^{T} x$ is constant on the affine space $\bar{x}(B)+L(B)$, then $\operatorname{Conv}\left(P(B) \cap F_{D\left(\pi, \pi_{0}^{1}, \pi_{0}^{2}\right)}\right)=L(B)+\operatorname{Conv}\left(P^{\prime}(B) \cap F_{D\left(\pi, \pi_{0}^{1}, \pi_{0}^{2}\right)}\right)$.

Proof. (i) Let $x^{1}, x^{2} \in \bar{x}(B)+L(B)$ satisfy $\pi^{T} x^{1} \neq \pi^{T} x^{2}$, and let $l:=x^{1}-x^{2} \in L(B)$. We have $\pi^{T} l \neq 0$. Let $x^{\prime}$ be a point in $P(B)$ satisfying $\left.\pi^{T} x^{\prime} \in\right] \pi_{0}^{1}, \pi_{0}^{2}\left[\right.$. The line $x^{\prime}+\alpha l$, $\alpha \in \mathbb{R}$, is contained in $P(B)$. Since $\pi^{T} l \neq 0$, the point $x^{\prime}$ can be expressed as a convex combination of two points on this line that satisfy the disjunction $D\left(\pi, \pi_{0}^{1}, \pi_{0}^{2}\right)$.

(ii) Now suppose $\pi^{T} x$ is constant on $\bar{x}(B)+L(B)$. This implies $\pi^{T} l=0$ for all $l \in L(B)$. Given a positive integer $k$, define $\Lambda^{k}:=\left\{\lambda \in \mathbb{R}^{k}: \lambda \geq 0_{k}, \sum_{j=1}^{k} \lambda_{j}=1\right\}$. We have 


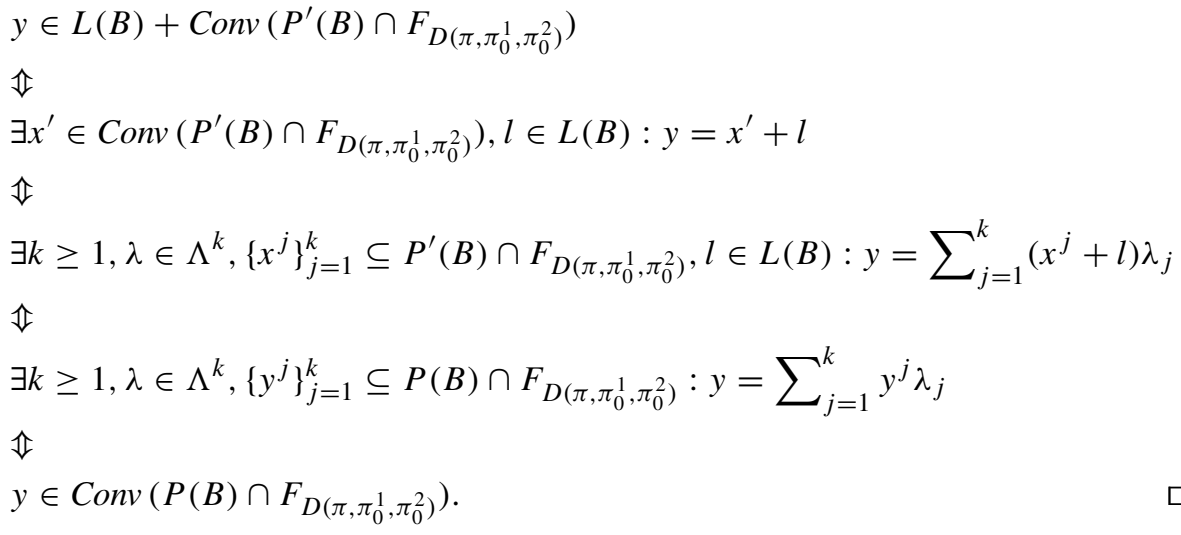

Combining Lemma 9 with Lemma 4 in Sect. 3.2 gives Lemma 1 in Sect. 2.1.

\section{Corollary 2. Lemma 1 holds.}

Proof. (i) If $\pi^{T} x$ is not constant on $\bar{x}(B)+L(B)$, we are done (Lemma 9(i)). So we assume $\pi^{T} x$ is constant on $\bar{x}(B)+L(B)$. Let $x^{\prime} \in \bar{x}(B)+L(B)$ satisfy $x^{\prime} \in$ $F_{D\left(\pi, \pi_{0}^{1}, \pi_{0}^{2}\right)}$. Since $x^{\prime} \in F_{D\left(\pi, \pi_{0}^{1}, \pi_{0}^{2}\right)}$, we know $\bar{x}(B)+L(B) \subseteq F_{D\left(\pi, \pi_{0}^{1}, \pi_{0}^{2}\right)}$. In particular $\bar{x}(B) \in F_{D\left(\pi, \pi_{0}^{1}, \pi_{0}^{2}\right)}$. Now, since $P^{\prime}(B)$ is a simple conic polyhedron, Lemma 4(i) gives $\operatorname{Conv}\left(P^{\prime}(B) \cap F_{D\left(\pi, \pi_{0}^{1}, \pi_{0}^{2}\right)}\right)=P^{\prime}(B)$. Combining this fact with Lemma 9(ii) gives $\operatorname{Conv}\left(P(B) \cap F_{D\left(\pi, \pi_{0}^{1}, \pi_{0}^{2}\right)}\right)=P(B)$.

(ii) Suppose $\left.\pi^{T} x \in\right] \pi_{0}^{1}, \pi_{0}^{2}\left[\right.$ for every $x \in \bar{x}(B)+L(B)$. Then $\pi^{T} x$ is constant on $\bar{x}(B)+L(B)$, and $\pi^{T} l=0$ holds for all $l \in L(B)$. In fact, if $L(B)=\{0\}$, the result is obvious; otherwise, $L(B)$ is a linear space, and we must have $\pi^{T} l=0$ to avoid that $x+\alpha l$ satisfies the disjunction for some $\alpha \in \mathbb{R}$.

Now suppose $\left(\delta\left(\pi, \pi_{0}\right)\right)^{T} l \neq 0$ for some $l \in L(B)$. Let $x^{\prime} \in P(B) \cap F_{D\left(\pi, \pi_{0}^{1}, \pi_{0}^{2}\right)}$ be arbitrary, and let $l^{\prime} \in L(B)$ satisfy $\left(\delta\left(\pi, \pi_{0}\right)\right)^{T} l^{\prime}<0$. The point $x^{\prime}+\alpha l^{\prime}$ is in $P(B) \cap F_{D\left(\pi, \pi_{0}^{1}, \pi_{0}^{2}\right)}$ and violates the inequality $\left(\delta\left(\pi, \pi_{0}\right)\right)^{T} x \geq \delta_{0}\left(\pi, \pi_{0}\right)$ for $\alpha>0$ sufficiently large. Hence $\left(\delta\left(\pi, \pi_{0}\right)\right)^{T} l=0$ for all $l \in L(B)$.

Since $P^{\prime}(B)$ is a simple conic polyhedron, Lemma 4(ii) gives $\operatorname{Conv}\left(P^{\prime}(B) \cap\right.$ $\left.F_{D\left(\pi, \pi_{0}^{1}, \pi_{0}^{2}\right)}\right)=\left\{x \in P^{\prime}(B):(15)\right\}$. Combining this with Lemma 9(ii) gives the identity $\operatorname{Conv}\left(P(B) \cap F_{D\left(\pi, \pi_{0}^{1}, \pi_{0}^{2}\right)}\right)=L(B)+\left\{x \in P^{\prime}(B):(15)\right\}$. Finally, since $\left(\delta\left(\pi, \pi_{0}\right)\right)^{T} l=$ 0 for all $l \in L(B)$, we get $\left\{x \in P^{\prime}(B):(15)\right\}+L(B)=\left\{x \in P^{\prime}(B)+L(B):(15)\right\}=$ $\{x \in P(B):(15)\}$.

By combining Lemma 8 and Lemma 9 we can prove

Corollary 3. $S C(B)$ is a polyhedron.

Proof. Let $\Pi_{V}^{n}\left(N_{I}\right):=\left\{\left(\pi, \pi_{0}\right) \in \Pi^{n}\left(N_{I}\right): \pi^{T} x \in\right] \pi_{0}, \pi_{0}+1[, \forall x \in \bar{x}(B)+L(B)\}$ denote the set of violated split disjunctions for $P(B)$. The split closure of $P(B)$ is given by $\operatorname{SC}(B)=\cap_{\left(\pi, \pi_{0}\right) \in \Pi_{V}^{n}\left(N_{I}\right)} \operatorname{Conv}\left(P(B) \cap F_{D\left(\pi, \pi_{0}\right)}\right)$. Using Lemma 9 gives $\operatorname{SC}(B)=$ 
$L(B)+\cap_{\left(\pi, \pi_{0}\right) \in \Pi_{V}^{n}\left(N_{I}\right)} \operatorname{Conv}\left(P^{\prime}(B) \cap F_{D\left(\pi, \pi_{0}\right)}\right)$. Since $P^{\prime}(B)$ is a simple conic polyhedron, Lemma 8 implies that $\cap_{\left(\pi, \pi_{0}\right) \in \Pi_{V}^{n}\left(N_{I}\right)} \operatorname{Conv}\left(P^{\prime}(B) \cap F_{D\left(\pi, \pi_{0}\right)}\right)$ is a polyhedron.

Given $B^{\prime} \in \mathcal{B}_{k}^{*}$, where $0<k<r$, and a general split disjunction $D\left(\pi, \pi_{0}^{1}, \pi_{0}^{2}\right)$ satisfying $\left.\pi^{T} y \in\right] \pi_{0}^{1}, \pi_{0}^{2}\left[, \forall y \in \bar{x}\left(B^{\prime}\right)+L\left(B^{\prime}\right)\right.$, an intersection cut can be derived from $B^{\prime}$ and $D\left(\pi, \pi_{0}^{1}, \pi_{0}^{2}\right)$. A question is how the intersection cut derived from $B^{\prime}$ and $D\left(\pi, \pi_{0}^{1}, \pi_{0}^{2}\right)$ relates to intersection cuts derived from $D\left(\pi, \pi_{0}^{1}, \pi_{0}^{2}\right)$ and sets $B \in \mathcal{B}_{r}^{*}$. The next lemma shows that the intersection cut derived from $B^{\prime}$ and $D\left(\pi, \pi_{0}^{1}, \pi_{0}^{2}\right)$ is equivalent to any intersection cut derived from $D\left(\pi, \pi_{0}^{1}, \pi_{0}^{2}\right)$ and any $B \in \mathcal{B}_{r}^{*}$ satisfying $B^{\prime} \subset B$, i.e., the size of $B^{\prime}$ is not important.

Lemma 10. Let $B^{\prime} \in \mathcal{B}_{k}^{*}$, where $0<k<r$, satisfy $\left.\pi^{T} y \in\right] \pi_{0}^{1}, \pi_{0}^{2}[$ for all $y \in$ $\bar{x}\left(B^{\prime}\right)+L\left(B^{\prime}\right)$ and $\operatorname{Conv}\left(P\left(B^{\prime}\right) \cap F_{D\left(\pi, \pi_{0}^{1}, \pi_{0}^{2}\right)}\right)=\left\{x \in P\left(B^{\prime}\right): \delta^{T} x \geq \delta_{0}\right\}$. Then $\operatorname{Conv}\left(P(B) \cap F_{D\left(\pi, \pi_{0}^{1}, \pi_{0}^{2}\right)}\right)=\left\{x \in P(B): \delta^{T} x \geq \delta_{0}\right\}$ for any $B \in \mathcal{B}_{r}^{*}$ satisfying $B \supset B^{\prime}$.

Proof. Let $B^{\prime}$ and $B$ be as stated. Wlog we assume that $B$ and $B^{\prime}$ only differ in one element, i.e., $B=B^{\prime} \cup\{q\}$, where $q \in M \backslash B^{\prime}$. Using the notation in Sect. 2.1, we can choose $r^{j}(B)=r^{j}\left(B^{\prime}\right)$ for $j \in B^{\prime}$ and $\bar{x}(B)=\bar{x}\left(B^{\prime}\right)$. This implies $\alpha_{j}\left(\pi, \pi_{0}^{1}, \pi_{0}^{2}, B\right)=$ $\alpha_{j}\left(\pi, \pi_{0}^{1}, \pi_{0}^{2}, B^{\prime}\right)$ for $j \in B^{\prime}$, i.e., the intersection cut associated with $B$ only differs from the intersection cut associated with $B^{\prime}$ in the coefficient corresponding to $q$. For simplicity, let $\alpha_{j}:=\alpha_{j}\left(\pi, \pi_{0}^{1}, \pi_{0}^{2}, B\right)$ for $j \in B$ and $\alpha_{j}^{\prime}:=\alpha_{j}\left(\pi, \pi_{0}^{1}, \pi_{0}^{2}, B^{\prime}\right)$ for $j \in B^{\prime}$.

By assumption $\left\{x \in P\left(B^{\prime}\right): \delta^{T} x \geq \delta_{0}\right\}=\operatorname{Conv}\left(P\left(B^{\prime}\right) \cap F_{D\left(\pi, \pi_{0}^{1}, \pi_{0}^{2}\right)}\right)$. Now $P(B) \subseteq P\left(B^{\prime}\right)$, so $\operatorname{Conv}\left(P(B) \cap F_{D\left(\pi, \pi_{0}^{1}, \pi_{0}^{2}\right)} \subseteq \operatorname{Conv}\left(P\left(B^{\prime}\right) \cap F_{D\left(\pi, \pi_{0}^{1}, \pi_{0}^{2}\right)}\right)\right.$. This implies that the inequality $\delta^{T} x \geq \delta_{0}$ is valid for $\operatorname{Conv}\left(P(B) \cap F_{D\left(\pi, \pi_{0}^{1}, \pi_{0}^{2}\right)}\right)$. Therefore, $\operatorname{Conv}\left(P(B) \cap F_{D\left(\pi, \pi_{0}^{1}, \pi_{0}^{2}\right)}\right) \subseteq\left\{x \in P(B): \delta^{T} x \geq \delta_{0}\right\}$.

For the other direction suppose $y \in\left\{x \in P(B): \delta^{T} x \geq \delta_{0}\right\}$. Since $P(B) \subseteq P\left(B^{\prime}\right)$ and $y \in P(B)$, we have $y \in P\left(B^{\prime}\right)$. Also, since $\delta^{T} y \geq \delta_{0}$ and $y \in P\left(B^{\prime}\right)$, we have $y \in$ $\operatorname{Conv}\left(P\left(B^{\prime}\right) \cap F_{D\left(\pi, \pi_{0}^{1}, \pi_{0}^{2}\right)}\right)$. This implies that $y$ satisfies the intersection cut associated with $B^{\prime}$ and $D\left(\pi, \pi_{0}^{1}, \pi_{0}^{2}\right)$, i.e., the inequality $\sum_{i \in B^{\prime}}\left(b_{i}-a_{i .}^{T} y\right) / \alpha_{i}^{\prime} \geq 1$ holds. Finally, using the facts $a_{q .}^{T} y \leq b_{q}, \alpha_{j}=\alpha_{j}^{\prime}$ for $j \in B^{\prime}$ and $\alpha_{q} \geq 0$ gives $\sum_{i \in B}\left(b_{i}-a_{i .}^{T} y\right) / \alpha_{i} \geq 1$, i.e., $y$ satisfies the intersection cut associated with $B$ and $D\left(\pi, \pi_{0}^{1}, \pi_{0}^{2}\right)$. From Lemma 1 it follows that $y \in \operatorname{Conv}\left(P(B) \cap F_{D\left(\pi, \pi_{0}^{1}, \pi_{0}^{2}\right)}\right)$.

\section{Split closure characterization}

In this section we give a geometric argument for the validity of Theorem 1 . As mentioned in Sect. 2 this leads to a characterization of the split closure in terms of intersection cuts (Corollary 1).

Throughout this section $D\left(\pi, \pi_{0}^{1}, \pi_{0}^{2}\right)$ denotes a general split disjunction, where $\pi \in \mathbb{R}^{n}$ and $\pi_{0}^{1}<\pi_{0}^{2}$. The following notation will be convenient. Define $P_{1}:=$ 
$P \cap\left\{x \in \mathbb{R}^{n}: \pi^{T} x \leq \pi_{0}^{1}\right\}$ and $P_{2}:=P \cap\left\{x \in \mathbb{R}^{n}: \pi^{T} x \geq \pi_{0}^{2}\right\}$ to be the two subsets of $P$ that satisfy the disjunction $D\left(\pi, \pi_{0}^{1}, \pi_{0}^{2}\right)$. Furthermore, given $B \in \mathcal{B}_{r}^{*}$, define $P_{1}(B):=P(B) \cap\left\{x \in \mathbb{R}^{n}: \pi^{T} x \leq \pi_{0}^{1}\right\}$ and $P_{2}(B):=P(B) \cap\left\{x \in \mathbb{R}^{n}: \pi^{T} x \geq \pi_{0}^{2}\right\}$. The following inclusion is easy to prove.

\section{Lemma 11.}

$$
\operatorname{Conv}\left(P_{1} \cup P_{2}\right) \subseteq \bigcap_{B \in \mathcal{B}_{r}^{*}} \operatorname{Conv}\left(P_{1}(B) \cup P_{2}(B)\right) .
$$

Proof. Let $B \in \mathcal{B}_{r}^{*}$. Clearly $P_{1} \cup P_{2} \subseteq P_{1}(B) \cup P_{2}(B)$. Convexifying both sides gives $\operatorname{Conv}\left(P_{1} \cup P_{2}\right) \subseteq \operatorname{Conv}\left(P_{1}(B) \cup P_{2}(B)\right)$. Since this holds for all $B \in \mathcal{B}_{r}^{*}$, the result follows.

The remainder of this section is divided into two parts. The focus is on proving the other inclusion $\operatorname{Conv}\left(P_{1} \cup P_{2}\right) \supseteq \bigcap_{B \in \mathcal{B}_{r}^{*}} \operatorname{Conv}\left(P_{1}(B) \cup P_{2}(B)\right)$. In Sect. 4.1 we consider the main case that $P, P_{1}$ and $P_{2}$ are all full-dimensional. In Sect. 4.2 all remaining cases are considered.

\subsection{Main case: $P_{1}, P_{2}$ and $P$ full-dimensional}

In this section we assume that the sets $P, P_{1}$ and $P_{2}$ are full-dimensional. We will show that every facet defining inequality for $\operatorname{Conv}\left(P_{1} \cup P_{2}\right)$ is valid for $\cap_{B \in \mathcal{B}_{r}^{*}} \operatorname{Conv}\left(P_{1}(B) \cup\right.$ $\left.P_{2}(B)\right)$. This will show the direction $\operatorname{Conv}\left(P_{1} \cup P_{2}\right) \supseteq \cap_{B \in \mathcal{B}_{r}^{*}} \operatorname{Conv}\left(P_{1}(B) \cup P_{2}(B)\right)$. Let $\delta^{T} x \leq \delta_{0}$ be an arbitrary facet defining inequality for $\operatorname{Conv}\left(P_{1} \cup P_{2}\right)$. Because $\cap_{B \in \mathcal{B}_{r}^{*}} \operatorname{Conv}\left(P_{1}(B) \cup P_{2}(B)\right) \subseteq P$, we can assume that $\delta^{T} x \leq \delta_{0}$ is not valid for $P$.

To show $\delta^{T} x \leq \delta_{0}$ is valid for $\cap_{B \in \mathcal{B}_{r}^{*}} \operatorname{Conv}\left(P_{1}(B) \cup P_{2}(B)\right)$, it suffices to prove that there exists $\bar{B} \in \mathcal{B}_{r}^{*}$ such that $\delta^{T} x \leq \delta_{0}$ is valid for $\operatorname{Conv}\left(P_{1}(\bar{B}) \cup P_{2}(\bar{B})\right)$. In particular, we prove the stronger fact that there exists $\bar{B} \in \mathcal{B}_{r}^{*}$ such that $\operatorname{Conv}\left(P_{1}(\bar{B}) \cup P_{2}(\bar{B})\right)=$ $\left\{x \in P(\bar{B}): \delta^{T} x \leq \delta_{0}\right\}$, i.e., $\delta^{T} x \leq \delta_{0}$ can be obtained as an intersection cut. Lemma 10 implies that it suffices to find $\bar{B}$ in $\mathcal{B}_{k}^{*}$, where $0<k<r$, such that $\operatorname{Conv}\left(P_{1}(\bar{B}) \cup\right.$ $\left.P_{2}(\bar{B})\right)=\left\{x \in P(\bar{B}): \delta^{T} x \leq \delta_{0}\right\}$.

Let $F:=\left\{x \in \operatorname{Conv}\left(P_{1} \cup P_{2}\right): \delta^{T} x=\delta_{0}\right\}$ be the facet of $\operatorname{Conv}\left(P_{1} \cup P_{2}\right)$ defined by $\delta^{T} x \leq \delta_{0}$. Furthermore, let $F_{1}:=F \cap P_{1}$ and $F_{2}:=F \cap P_{2}$. The assumptions that $P_{1}$ and $P_{2}$ are non-empty and $\delta^{T} x \leq \delta_{0}$ is not valid for $P$ imply $F_{1} \neq \emptyset$ and $F_{2} \neq \emptyset$. We now give some basic properties of the sets $F, F_{1}$ and $F_{2}$ that we derived from the general split disjunction $D\left(\pi, \pi_{0}^{1}, \pi_{0}^{2}\right)$.

Lemma 12. (i) $F=\operatorname{Conv}\left(F_{1} \cup F_{2}\right)$.

(ii) For $k \in\{1,2\}$ we have $F_{k} \subseteq\left\{x \in \mathbb{R}^{n}: \pi^{T} x=\pi_{0}^{k}\right\}$, i.e., $F_{k}$ is contained in the hyperplane $\pi^{T} x=\pi_{0}^{k}$.

Proof. (i) is trivial, so we only prove (ii). Wlog let $k=1$. Assume there exists $x^{1} \in F_{1}$ satisfying $\pi^{T} x^{1}<\pi_{0}^{1}$. Note that $\delta^{T} x^{1}=\delta_{0}$. Since $\delta^{T} x \leq \delta_{0}$ is not valid for $P$, there exists $x^{2} \in P \backslash\left(P_{1} \cup P_{2}\right)$ such that $\delta^{T} x^{2}>\delta_{0}$. Let $z(\lambda):=x^{1} \lambda+(1-\lambda) x^{2}$, where $\lambda \in[0,1]$. Observe that $\delta^{T} z(\lambda)>\delta_{0}$ and $z(\lambda) \in P$ for all $\left.\left.\lambda \in\right] 0,1\right]$. By choosing $\lambda$ sufficiently close to zero, we have $\pi^{T} z(\lambda)<\pi_{0}^{1}$ and $\delta^{T} z(\lambda)>\delta_{0}$. For such a $\lambda$, $z(\lambda) \in P_{1}$ and $\delta^{T} z(\lambda)>\delta_{0}$. This contradicts the validity of $\delta^{T} x \leq \delta_{0}$ for $P_{1}$. 
Let $k \in\{1,2\}$ be arbitrary. Observe that, since $\delta^{T} x \leq \delta_{0}$ is valid for $P_{k}$ and $F_{k}=$ $\left\{x \in P_{k}: \delta^{T} x=\delta_{0}\right\}, F_{k}$ is a face of $P_{k}$. Let $d_{k}$ be the dimension of the face $F_{k}$ of $P_{k}$. We have $F_{k}=P_{k} \cap \operatorname{Aff}\left(F_{k}\right)$, where $\operatorname{Aff}\left(F_{k}\right)$ denotes the affine hull of $F_{k}$. The set $\bar{B}$ satisfying $\operatorname{Conv}\left(P_{1}(\bar{B}) \cup P_{2}(\bar{B})\right)=\left\{x \in P(\bar{B}): \delta^{T} x \leq \delta_{0}\right\}$ that we are about to construct is obtained from constraints of $P$ that can be used to describe $A f f\left(F_{1}\right)$ and $A f f\left(F_{2}\right)$. The following crucial lemma provides the constraints for the set $\bar{B}$.

Lemma 13. For $k \in\{1,2\}$, there exists $B_{k} \in \mathcal{B}_{n-d_{k}-1}^{*}$ such that

(i) $\operatorname{Aff}\left(F_{k}\right)=\left\{x \in \mathbb{R}^{n}: a_{i .}^{T} x=b_{i}, \forall i \in B_{k}, \pi^{T} x=\pi_{0}^{k}\right\}$, i.e., Aff $\left(F_{k}\right)$ is the intersection of the hyperplanes $\pi^{T} x=\pi_{0}^{k}$ and $a_{i}^{T} x=b_{i}$ for $i \in B_{k}$.

(ii) There exist $\bar{u}_{0}>0$ and $\bar{u}_{i} \geq 0$ for $i \in B_{1}$ such that $\delta=\bar{u}_{0} \pi+\sum_{i \in B_{1}} \bar{u}_{i} a_{i}$ and $\delta_{0}=\bar{u}_{0} \pi_{0}^{1}+\sum_{i \in B_{1}} \bar{u}_{i} b_{i}$.

(iii) There exist $\bar{v}_{0}>0$ and $\bar{v}_{i} \geq 0$ for $i \in B_{2}$ such that $\delta=\sum_{i \in B_{2}} \bar{v}_{i} a_{i .}-\bar{v}_{0} \pi$ and $\delta_{0}=\sum_{i \in B_{2}} \bar{v}_{i} b_{i}-\bar{v}_{0} \pi_{0}^{2}$.

Proof. Wlog let $k=1$. Consider the linear program (PLP)

$$
\begin{aligned}
& \max \quad \delta^{T} x \\
\text { s.t. } & a_{i .}^{T} x \leq b_{i}, \quad \forall i \in M, \quad\left(u_{i}\right) \\
\pi^{T} x \leq \pi_{0}^{1}, & \left(u_{0}\right)
\end{aligned}
$$

the problem of maximizing $\delta^{T} x$ over $P_{1}$. Since $\delta^{T} x \leq \delta_{0}$ is valid for $P_{1}$, and $F_{1}$ is the set of points in $P_{1}$ satisfying $\delta^{T} x=\delta_{0}, F_{1}$ is the set of optimal solutions to ( $P L P$ ). The dual of $(P L P)$ is the problem $(D L P)$ given by

$$
\begin{aligned}
\min u^{T} b+\pi_{0}^{1} u_{0} & \\
\text { s.t. } \sum_{i \in M} a_{i} u_{i}+\pi u_{0} & =\delta, \\
u & \geq 0_{m}, \\
u_{0} & \geq 0 .
\end{aligned}
$$

Let $\bar{x}$ be an optimal basic feasible solution to $(P L P)$, and let $\left(\bar{u}, \bar{u}_{0}\right)$ be a corresponding optimal basic feasible solution to $(D L P)$. Since the optimal objective value to $(D L P)$ is $\delta_{0}$, we have $\delta_{0}=b^{T} \bar{u}+\pi_{0}^{1} \bar{u}_{0}$.

If $\bar{u}_{0}=0$, then $\sum_{i \in M} a_{i} \bar{u}_{i}=\delta$ and $\sum_{i \in M} b_{i} \bar{u}_{i}=\delta_{0}$, which implies that $\delta^{T} x \leq \delta_{0}$ is valid for $P$ since $\bar{u} \geq 0_{m}$ which contradicts our assumption. Hence we must have $\bar{u}_{0}>0$, which means that $u_{0}$ is basic.

Let $\tilde{B}_{1}:=\left\{i \in M: u_{i}\right.$ basic $\}$. Since the variables $\left\{u_{i}\right\}_{i \in \tilde{B}_{1}} \cup\left\{u_{0}\right\}$ are all basic, the vectors $\left\{a_{i}\right\}_{i \in \tilde{B}_{1}} \cup\{\pi\}$ are linearly independent.

Let $M_{1}^{=}:=\left\{i \in M: a_{i}^{T} x=b_{i}, \forall x \in F_{1}\right\}$ denote the constraints of $P$ satisfied at equality by all points in $\dot{F}_{1}$. We will prove by contradiction that if $i \in M \backslash M_{1}^{=}$ then $\bar{u}_{i}=0$. Suppose there exists $k \in M \backslash M_{1}^{=}$satisfying $\bar{u}_{k}>0$. Consider an inner point $x^{I}$ of $F_{1}$, i.e., $x^{I}$ satisfies $\pi^{T} x^{I}=\pi_{0}^{1}, a_{i .}^{T} x^{I}=b_{i}$ for $i \in M_{1}^{=}$and $a_{i}^{T} x^{I}<b_{i}$ for $i \in M \backslash M_{1}^{=}$. Since $\delta=\sum_{i \in M} a_{i} \cdot \bar{u}_{i}+\pi \bar{u}_{0}$, we have $\delta^{T} x^{I}=\sum_{i \in M} a_{i .}^{T} x^{I} \bar{u}_{i}+\pi^{T} x^{I} \bar{u}_{0}<$ 
$\sum_{i \in M} b_{i} \bar{u}_{i}+\pi_{0}^{1} \bar{u}_{0}=\delta_{0}$, which contradicts $\delta^{T} x=\delta_{0}$ for all $x \in F_{1}$. Therefore we have $\bar{u}_{i}=0$ for all $i \in M \backslash M_{1}^{=}$.

By choosing a maximal subset $B_{1}$ of $M_{1}^{=}$such that $B_{1} \supseteq \tilde{B}_{1} \cap M_{1}^{=}$and the vectors $\{\pi\} \cup\left\{a_{i .}\right\}_{i \in B_{1}}$ are linearly independent, (i) and (ii) are satisfied. This proves (ii). The proof of (iii) is similar to the proof of (ii).

Let $B_{1}$ and $B_{2}$ be as in Lemma 13, and define $\bar{B}:=B_{1} \cup B_{2}$. We will show that the vectors $\left\{a_{i}\right\}_{i \in \bar{B}}$ are linearly independent (Lemma 16) and $\operatorname{Conv}\left(P_{1}(\bar{B}) \cup P_{2}(\bar{B})\right)=$ $\left\{x \in P(\bar{B}): \delta^{T} x \leq \delta_{0}\right\}$ (Lemma 19). This will finish the proof of Theorem 1 for the main case. For $k=1,2$, define $A^{k}:=\left\{x \in \mathbb{R}^{n}: a_{i}^{T} x=b_{i}, \forall i \in B_{k}\right\}$ to be the affine space defined by $B_{k}$. First, we prove the following lemma that describes how points in $A^{1}$ and $A^{2}$ are positioned relative to the hyperplanes $\pi^{T} x=\pi_{0}^{1}, \pi^{T} x=\pi_{0}^{2}$ and $\delta^{T} x=\delta_{0}$.

Lemma 14. For $x^{1} \in A^{1}$ and $x^{2} \in A^{2}$,

(i) $\pi^{T} x^{1}<\pi_{0}^{1} \Longleftrightarrow \delta^{T} x^{1}<\delta_{0}$,

$\pi^{T} x^{1}=\pi_{0}^{1} \Longleftrightarrow \delta^{T} x^{1}=\delta_{0}$ and

$\pi^{T} x^{1}>\pi_{0}^{1} \Longleftrightarrow \delta^{T} x^{1}>\delta_{0}$.

(ii) $\pi^{T} x^{2}>\pi_{0}^{2} \Longleftrightarrow \delta^{T} x^{2}<\delta_{0}$,

$\pi^{T} x^{2}=\pi_{0}^{2} \Longleftrightarrow \delta^{T} x^{2}=\delta_{0}$ and

$\pi^{T} x^{2}<\pi_{0}^{2} \Longleftrightarrow \delta^{T} x^{2}>\delta_{0}$.

Proof. By symmetry we only need to prove (i). Let $x^{1} \in A^{1}$, and let $\left\{\bar{u}_{i}\right\}_{i \in B_{1}}$ and $\bar{u}_{0}>0$ be as in Lemma 13. Since $a_{i .}^{T} x=b_{i}$ for all $x \in A^{1}, \sum_{i \in B_{1}} \bar{u}_{i} a_{i .}^{T} x$ is a constant on $A^{1}$ that is equal to $c:=\sum_{i \in B_{1}} \bar{u}_{i} b_{i}$. We conclude from Lemma 13 that $\delta_{0}=c+\bar{u}_{0} \pi_{0}^{1}$ and $\delta^{T} x=c+\bar{u}_{0} \pi^{T} x$ for all $x \in A^{1}$.

Now we have $\delta^{T} x^{1}<\delta_{0} \Longleftrightarrow \bar{u}_{0} \pi^{T} x^{1}+c<\delta_{0} \Longleftrightarrow \pi^{T} x^{1}<\pi_{0}^{1}$. Also, we have $\delta^{T} x^{1}=\delta_{0} \Longleftrightarrow \bar{u}_{0} \pi^{T} x^{1}+c=\delta_{0} \Longleftrightarrow \pi^{T} x^{1}=\pi_{0}^{1}$.

Now, we show that, if $B_{1}$ and $B_{2}$ are as in Lemma 13, then the vectors $\left\{a_{i}\right\}_{i \in B_{1} \cup B_{2}}$ are linearly independent. We will show $r\left(B_{1} \cup B_{2}\right)=\left|B_{1}\right|+\left|B_{2}\right|$. Observe that this is equivalent to showing that the set $L\left(B_{1}\right) \cap L\left(B_{2}\right)$ is of dimension $n-\left|B_{1}\right|-\left|B_{2}\right|$, where $L\left(B_{k}\right):=\left\{x \in \mathbb{R}^{n}: a_{i .}^{T} x=0, \forall i \in B_{k}\right\}$ for $k=1,2$ are as defined in Sect. 2.1.

Lemma 15. The set $L\left(B_{1}\right) \cap L\left(B_{2}\right) \cap\left\{x \in \mathbb{R}^{n}: \pi^{T} x=0\right\}$ is of dimensionn-|B $|-| B_{2} \mid$.

Proof. Let $\operatorname{Aff}\left(F_{1}\right)=L_{1}+y^{1}$ and $\operatorname{Aff}\left(F_{2}\right)=L_{2}+y^{2}$, where $y^{1} \in \operatorname{Aff}\left(F_{1}\right), y^{2} \in$ $\operatorname{Aff}\left(F_{2}\right)$, and $L_{1}$ and $L_{2}$ are the linear spaces parallel to $\operatorname{Aff}\left(F_{1}\right)$ and $\operatorname{Aff}\left(F_{2}\right)$ respectively. Observe that $L\left(B_{1}\right) \cap L\left(B_{2}\right) \cap\left\{x \in \mathbb{R}^{n}: \pi^{T} x=0\right\}=L_{1} \cap L_{2}$. Hence we need to show $\operatorname{dim}\left(L_{1} \cap L_{2}\right)=n-\left|B_{1}\right|-\left|B_{2}\right|$.

Let $\left\{x^{j}\right\}_{j=1}^{n} \subseteq F_{1} \cup F_{2}$ be affinely independent and chosen such that the first $d_{1}+1$ points are in $F_{1}$, i.e., Aff $\left(\left\{x^{j}\right\}_{j=1}^{d_{1}+1}\right)=\operatorname{Aff}\left(F_{1}\right)$. Necessarily, the remaining $\left(n-d_{1}-1\right)$ points are in $F_{2}$, i.e., $\left\{x^{j}\right\}_{j=d_{1}+2}^{n} \subseteq F_{2}$. Define $S_{1}:=A f f\left(\left\{x^{j}\right\}_{j=d_{1}+2}^{n}\right)-y^{2}$. We have $\operatorname{dim}\left(S_{1}\right)=n-d_{1}-2$. Furthermore, since $S_{1} \subseteq L_{2}$ and $\operatorname{dim}\left(S_{1}\right)=n-d_{1}-2$, we have $d_{2} \geq n-d_{1}-2$. Observe that $n-\left|B_{1}\right|-\left|B_{2}\right|=d_{2}-\left(n-d_{1}-2\right) \geq 0$. 
First suppose $d_{2}-\left(n-d_{1}-2\right)=0$. Then $L_{2}=S_{1}$. Since Aff $\left(\left\{x^{j}\right\}_{j=1}^{d_{1}+1}\right)-y^{1}=L_{1}$, the vectors in $L_{1}$ are linearly independent from those in $L_{2}=S_{1}$, so that $L_{1} \cap L_{2}=\left\{0_{n}\right\}$. Therefore, $\operatorname{dim}\left(L_{1} \cap L_{2}\right)=0=d_{2}-\left(n-d_{1}-2\right)=n-\left|B_{1}\right|-\left|B_{2}\right|$.

Now suppose $d_{2}-\left(n-d_{1}-2\right)>0$. Let $d_{2}^{r}:=d_{2}-\left(n-d_{1}-2\right)$. Choose $d_{2}^{r}$ vectors $\left\{z^{j}\right\}_{j=1}^{d_{2}^{r}}$ from $L_{2} \backslash S_{1}$ such that they, together with $S_{1}$, span $L_{2}$. Define $S_{2}:=\operatorname{Span}\left(\left\{z^{j}\right\}_{j=1}^{d_{2}^{r}}\right)$. By construction, we have $L_{2}=S_{1}+S_{2}$ and $S_{1} \cap S_{2}=\left\{0_{n}\right\}$. Furthermore we have $S_{1} \cap L_{1}=\left\{0_{n}\right\}$. From this it follows that $L_{1} \cap L_{2}=S_{2}$, so that $\operatorname{dim}\left(L_{1} \cap L_{2}\right)=d_{2}^{r}=n-\left|B_{1}\right|-\left|B_{2}\right|$.

By combining Lemma 14 and Lemma 15 we get

Lemma 16. Let $B_{1}$ and $B_{2}$ be as in Lemma 13. Then

(i) $B_{1} \cap B_{2}=\emptyset$,

(ii) The vectors $\left\{a_{i}\right\}_{i \in B_{1} \cup B_{2}}$ are linearly independent.

Proof. Lemma 14(i) implies that every solution to the system $a_{i}^{T} x=0, i \in B_{1}$ satisfying $\pi^{T} \bar{x} \neq 0$ is either in the set $\left\{x \in \mathbb{R}^{n}: \pi^{T} x<0, \delta^{T} x<0\right\}$ or in the set $\left\{x \in \mathbb{R}^{n}\right.$ : $\left.\pi^{T} x>0, \delta^{T} x>0\right\}$. Furthermore, Lemma 14(ii) implies every solution to the system $a_{i .}^{T} x=0, i \in B_{2}$ satisfying $\pi^{T} \bar{x} \neq 0$ is either in the set $\left\{x \in \mathbb{R}^{n}: \pi^{T} x>0, \delta^{T} x<0\right\}$ or in the set $\left\{x \in \mathbb{R}^{n}: \pi^{T} x<0, \delta^{T} x>0\right\}$.

From this we see that every solution to the system $a_{i .}^{T} x=0, i \in B_{1} \cup B_{2}$ must satisfy $\pi^{T} x=0$. This implies $L\left(B_{1}\right) \cap L\left(B_{2}\right)=L\left(B_{1}\right) \cap L\left(B_{2}\right) \cap\left\{x \in \mathbb{R}^{n}: \pi^{T} x=0\right\}$. By applying Lemma 15 we get that $L\left(B_{1}\right) \cap L\left(B_{2}\right)$ is of dimension $n-\left|B_{1}\right|-\left|B_{2}\right|$. Since the description of $L\left(B_{1}\right) \cap L\left(B_{2}\right)$ involves $\left|B_{1}\right|+\left|B_{2}\right|$ constraints, this implies both (i) and (ii).

In the remainder of this section we show $\operatorname{Conv}\left(P_{1}(\bar{B}) \cup P_{2}(\bar{B})\right)=\{x \in P(\bar{B})$ : $\left.\delta^{T} x \leq \delta_{0}\right\}$, where $\bar{B}:=B_{1} \cup B_{2}$. The following lemma shows the first inclusion.

Lemma 17. Let $B_{1}$ and $B_{2}$ be as in Lemma 13 , and let $\bar{B}:=B_{1} \cup B_{2}$. Then $\delta^{T} x \leq \delta_{0}$ is valid for $\operatorname{Conv}\left(P_{1}(\bar{B}) \cup P_{2}(\bar{B})\right)$.

Proof. We will show that $\delta^{T} x \leq \delta_{0}$ is valid for both $P_{1}\left(B_{1}\right)$ and $P_{2}\left(B_{2}\right)$. Since $P_{1}(\bar{B}) \subseteq$ $P_{1}\left(B_{1}\right)$ and $P_{2}(\bar{B}) \subseteq P_{2}\left(B_{2}\right)$, this implies that $\delta^{T} x \leq \delta_{0}$ is valid for $\operatorname{Conv}\left(P_{1}(\bar{B}) \cup\right.$ $\left.P_{2}(\bar{B})\right)$. Wlog we only show that $\delta^{T} x \leq \delta_{0}$ is valid for $P_{1}\left(B_{1}\right)$.

Recall that $P_{1}\left(B_{1}\right)=\left\{x \in \mathbb{R}^{n}: a_{i}^{\bar{T}} x \leq b_{i}, i \in B_{1}, \pi^{T} x \leq \pi_{0}^{1}\right\}$. We now derive a representation of $P_{1}\left(B_{1}\right)$ in terms of extreme points and extreme rays. Let $\bar{x}$ be a solution to the system $\pi^{T} x=\pi_{0}^{1}$ and $a_{i}^{T} x=b_{i}$ for all $i \in B_{1}$. Also, for $j \in B_{1}$, let $\bar{r}^{j}$ be a solution to the system $a_{i .}^{T} x=0, \forall i \in B_{1} \backslash\{j\}, a_{j}^{T} x=-1$ and $\pi^{T} x=0$. Finally, let $\bar{r}^{\pi}$ be a solution to the system $a_{i}^{T} x=0, \forall i \in B_{1}$, and $\pi^{T} x=-1$. We have $P_{1}\left(B_{1}\right)=\bar{x}+L^{\pi}\left(B_{1}\right)+$ Cone $\left(\left\{\bar{r}^{j}\right\}_{j \in B_{1}} \cup\left\{\bar{r}^{\pi}\right\}\right)$, where $L^{\pi}\left(B_{1}\right):=\left\{x \in \mathbb{R}^{n}: a_{i .}^{T} x=0, \forall i \in B_{1}, \pi^{T} x=0\right\}$.

Observe that $\bar{x}+L^{\pi}\left(B_{1}\right) \subseteq A^{1}$ (where $A^{1}:=\left\{x \in \mathbb{R}^{n}: a_{i}^{T} x=b_{i}, \forall i \in B_{1}\right\}$ as defined earlier). Furthermore $\pi^{T} x=\pi_{0}^{1}$ for every $x \in \bar{x}+L^{\pi}\left(B_{1}\right)$, which implies that $\delta^{T} x=\delta_{0}$ for all $x \in \bar{x}+L^{\pi}\left(B_{1}\right)$ (Lemma 14(i)). By using the scalars from Lemma 13(ii), we get $\delta^{T} \bar{r}^{j}=-\bar{u}_{j} \leq 0$ for all $j \in B_{1}$ and $\delta^{T} \bar{r}^{\pi}=-\bar{u}_{0}<0$. Since all the extreme rays $\bar{r}^{j}$ of $P_{1}\left(B_{1}\right)$ are such that $\delta^{T} \bar{r}^{j} \leq 0$ and $\delta^{T} \bar{x}=\delta_{0}$, we have that $\delta^{T} x \leq \delta_{0}$ is valid for $P_{1}\left(B_{1}\right)$. 
Lemma 17 proves $\operatorname{Conv}\left(P_{1}(\bar{B}) \cup P_{2}(\bar{B})\right) \subseteq\left\{x \in P(\bar{B}): \delta^{T} x \leq \delta_{0}\right\}$, where $\bar{B}=B_{1} \cup B_{2}$, i.e., that $\delta^{T} x \leq \delta_{0}$ is valid for $\operatorname{Conv}\left(P_{1}(\bar{B}) \cup P_{2}(\bar{B})\right)$. Combining this result with Lemma 10 and Lemma 11 finishes the proof of Theorem 1 for the main case. The remainder of this section is devoted to proving $\operatorname{Conv}\left(P_{1}(\bar{B}) \cup P_{2}(\bar{B})\right) \supseteq$ $\left\{x \in P(\bar{B}): \delta^{T} x \leq \delta_{0}\right\}$. To simplify notation, let $\bar{x}:=\bar{x}(\bar{B})$ and $\bar{r}^{j}:=r^{j}(\bar{B})$ for $j \in \overline{\bar{B}}$ (see Sect. 2.1). First we prove some basic properties of $P(\bar{B})$.

Lemma 18. Let $\bar{B}=B_{1} \cup B_{2}$, where $B_{1}$ and $B_{2}$ are as in Lemma 13 .

(i) $\left.\pi^{T} x \in\right] \pi_{0}^{1}, \pi_{0}^{2}\left[\right.$ for all $x \in A^{1} \cap A^{2}=\bar{x}+L(\bar{B})$.

(ii) $\pi^{T} \bar{r}^{j} \geq 0$ for all $j \in B_{1}$.

(iii) $\pi^{T} \bar{r}^{j} \leq 0$ for all $j \in B_{2}$.

Proof. (i) The proof is by contradiction. Let $y \in \bar{x}+L(\bar{B})$ and wlog assume $\pi^{T} y \leq \pi_{0}^{1}$. Since $y \in A^{1}$, Lemma 14(i), implies $\delta^{T} y \leq \delta_{0}$. However, since $y \in A^{2}$ and $\pi^{T} y \leq$ $\pi_{0}^{1}<\pi_{0}^{2}$, Lemma 14(ii) implies $\delta^{T} y>\delta_{0}$, which is a contradiction.

(ii)-(iii) We only prove (ii). We know $P\left(B_{1}\right)=A^{1}+$ Cone $\left(\left\{\bar{r}^{j}\right\}_{j \in B_{1}}\right)$. Let $j \in B_{1}$, and assume $\pi^{T} \bar{r}^{j}<0$ for a contradiction. We have $\bar{x}+\alpha \bar{r}^{j} \in A^{2}$ and $\bar{x}+\alpha \bar{r}^{j} \in$ $P(\bar{B}) \subseteq P\left(B_{1}\right)$ for every $\alpha \geq 0$. Let $\bar{\alpha}>0$ satisfy $\pi^{T}\left(\bar{x}+\bar{\alpha} \bar{r}^{j}\right)=\pi_{0}^{1}$. Lemma 14(ii) gives $\delta^{T}\left(\bar{x}+\bar{\alpha} \bar{r}^{j}\right)>\delta_{0}$. This contradicts the validity of $\delta^{T} x \leq \delta_{0}$ for $P_{1}\left(B_{1}\right)$.

Lemma 18(i) states that an intersection cut can be derived from $D\left(\pi, \pi_{0}^{1}, \pi_{0}^{2}\right)$ and $\bar{B}$ (Lemma 1). Lemma 18(ii) states that rays associated with elements of $B_{1}$ point in the direction of satisfaction of the term $\pi^{T} x \geq \pi_{0}^{2}$ of the disjunction $D\left(\pi, \pi_{0}^{1}, \pi_{0}^{2}\right)$, and Lemma 18(iii) states that rays associated with elements of $B_{2}$ point in the opposite direction.

To simplify notation, let $\bar{\alpha}_{j}:=\alpha_{j}\left(\pi, \pi_{0}^{1}, \pi_{0}^{2}, \bar{B}\right)$ denote the coefficient in the intersection cut associated with $\bar{B}$ and $D\left(\pi, \pi_{0}^{1}, \pi_{0}^{2}\right)$ for $j \in \bar{B}$. We are now able to prove $\operatorname{Conv}\left(P_{1}(\bar{B}) \cup P_{2}(\bar{B})\right)=\left\{x \in P(\bar{B}): \delta^{T} x \leq \delta_{0}\right\}$.

Lemma 19. Let $\bar{B}=B_{1} \cup B_{2}$, where $B_{1}$ and $B_{2}$ are as in Lemma 13. Then

(i) $\bar{\alpha}_{j}=\left(\delta_{0}-\delta^{T} \bar{x}\right) / \delta^{T} \bar{r}^{j}$ for $j \in \bar{B}$ satisfying $\delta^{T} \bar{r}^{j} \neq 0$.

(ii) $\operatorname{Conv}\left(P_{1}(\bar{B}) \cup P_{2}(\bar{B})\right)=\left\{x \in P(\bar{B}): \delta^{T} x \leq \delta_{0}\right\}$.

Proof. (i) Let $j \in \bar{B}$ satisfy $\pi^{T} \bar{r}^{j} \neq 0$. Wlog assume $\pi^{T} \bar{r}^{j}>0$, so $j \in B_{1}$ (Lemma 18(ii)). Choose $\bar{\alpha}_{j}$ such that $\pi^{T}\left(\bar{x}+\bar{\alpha}_{j} \bar{r}^{j}\right)=\pi_{0}^{2}$. Since $\bar{x}+\bar{\alpha}_{j} \bar{r}^{j} \in A^{2}$, we have $\delta^{T}\left(\bar{x}+\bar{\alpha}_{j} \bar{r}^{j}\right)=\delta_{0}$ (Lemma 14(ii)). Solving for $\bar{\alpha}_{j}$ gives (i). Notice that, since $\delta^{T} \bar{x}>\delta_{0}$ (Lemma 18(i) and Lemma 14), we have $\delta^{T} \bar{r}^{j}<0$.

(ii) We already proved $\operatorname{Conv}\left(P_{1}(\bar{B}) \cup P_{2}(\bar{B})\right) \subseteq\left\{x \in P(\bar{B}): \delta^{T} x \leq \delta_{0}\right\}$ (Lemma 17). Now suppose that $y \in P(\bar{B})$ satisfies $\delta^{T} y \leq \delta_{0}$. Since $y \in P(\bar{B})$, we may write $y=x^{\prime}+\sum_{j \in \bar{B}} \bar{r}^{j} s_{j}^{y}$, where $s_{j}^{y} \geq 0$ for $j \in \bar{B}$ denotes the slack in the $j^{\text {th }}$ constraint, i.e., $s_{j}^{y}=b_{j}-a_{j}^{T} y$, and $x^{\prime} \in \bar{x}+L(\bar{B})$. Multiplying with $\delta$ on both sides and deducting $\delta_{0}$ gives $\delta^{T} y-\delta_{0}=\sum_{j \in \bar{B}} \delta^{T} \bar{r}^{j} s_{j}^{y}+\delta^{T} x^{\prime}-\delta_{0}$. Since $\delta^{T} y \leq \delta_{0}$, we have $0 \geq \sum_{j \in \bar{B}} \delta^{T} \bar{r}^{j} s_{j}^{y}+\delta^{T} x^{\prime}-\delta_{0}$. Dividing with $\delta^{T} x^{\prime}-\delta_{0}>0$ on both sides gives $\sum_{j \in \bar{B}}$ $s_{j}^{y}\left(\delta^{T} \bar{r}^{j}\right) /\left(\delta_{0}-\delta^{T} x^{\prime}\right) \geq 1$.

Observe that Lemma 18(i) and the fact that $\bar{x}+L(\bar{B})=A^{1} \cap A^{2}$ is affine implies the linear function $\pi^{T} x$ is constant on $\bar{x}+L(\bar{B})$. Now, using Lemma 14, this implies the linear function $\delta^{T} x$ is constant on $\bar{x}+L(\bar{B})$. Therefore $\delta^{T} x^{\prime}=\delta^{T} \bar{x}$. 
Now using (i) we get $\sum_{j \in \bar{B}} s_{j}^{y} / \bar{\alpha}_{j} \geq 1$. Hence $y$ satisfies the intersection cut associated with $\bar{B}$ and the disjunction $D\left(\pi, \pi_{0}^{1}, \pi_{0}^{2}\right)$, which implies $y \in \operatorname{Conv}\left(P_{1}(\bar{B}) \cup P_{2}(\bar{B})\right)$.

\subsection{Reduction to the main case}

In this section, we consider the remaining cases, i.e., when $P$ or $P_{1}$ or $P_{2}$ is not fulldimensional. We distinguish between three cases, namely 1) One of the sets is empty 2) $\mathrm{P}$ is full-dimensional and 3) $\mathrm{P}$ is not full-dimensional. First we prove Theorem 1 when $P$ is empty.

Lemma 20. Theorem 1 holds when $P$ is empty.

Proof. If $P$ is empty, $\operatorname{Conv}\left(P \cap F_{D\left(\pi, \pi_{0}^{1}, \pi_{0}^{2}\right)}\right)=\emptyset$. We know $\cap_{B \in \mathcal{B}_{r}^{*}} P(B)=P$. Since $P(B)$ is convex, $\cap_{B \in \mathcal{B}_{r}^{*}} \operatorname{Conv}\left(P(B) \cap F_{D\left(\pi, \pi_{0}^{1}, \pi_{0}^{2}\right)} \subseteq \cap_{B \in \mathcal{B}_{r}^{*}} P(B)=\emptyset\right.$.

From now on we consider the cases in which $P$ is not empty. Next we consider the case where one of the sets $P_{1}$ and $P_{2}$ is empty. Wlog assume $P_{2}$ is empty.

Lemma 21. Theorem 1 holds when $P_{2}$ is empty.

Proof. Since $P_{2}=\emptyset, \operatorname{Conv}\left(P_{1} \cup P_{2}\right)=P_{1}=P \cap\left\{x: \pi^{T} x \leq \pi_{0}^{1}\right\}$. Hence $\pi^{T} x \leq \pi_{0}^{1}$ is valid for $\operatorname{Conv}\left(P_{1} \cup P_{2}\right)$. Furthermore, it is the only inequality needed to describe Conv $\left(P_{1} \cup P_{2}\right)$ besides the constraints of $P$. We just need to show that it can be obtained from a set $B \in \mathcal{B}_{r}^{*}$. Consider the linear program $z:=\max \left\{\pi^{T} x: x \in P\right\}$. We have $z<\pi_{0}^{2}$. By the linear programming duality theorem, there exists $B \in \mathcal{B}_{r}^{*}$ that satisfies $\pi=\sum_{i \in B} y_{i} a_{i}, z=\sum_{i \in B} y_{i} b_{i}$. and $y_{i} \geq 0$ for $i \in B$. Any $x \in P(B)$ satisfies $\pi^{T} x=\sum_{i \in B} y_{i} a_{i .}^{T} x \leq \sum_{i \in B} y_{i} b_{i} .=z$. Therefore, $P(B) \cap\left\{x: \pi^{T} x \geq \pi_{0}^{2}\right\}=\emptyset$ and $\operatorname{Conv}\left(P_{1}(B) \cup P_{2}(B)\right)=P_{1}(B)$ for this particular $B$.

Next we consider the case where $P$ is full-dimensional.

Lemma 22. Theorem 1 holds when $P$ is full-dimensional.

Proof. Because of Lemma 21, we can assume $P_{1}$ and $P_{2}$ are not empty. Choose $\epsilon>0$ such that $\pi_{0}^{1}+\epsilon<\pi_{0}^{2}-\epsilon$. Let $P_{1}^{\epsilon}:=P \cap\left\{x \in \mathbb{R}^{n}: \pi^{T} x \leq \pi_{0}^{1}+\epsilon\right\}$ and $P_{2}^{\epsilon}:=$ $P \cap\left\{x \in \mathbb{R}^{n}: \pi^{T} x \geq \pi_{0}^{2}-\epsilon\right\}$. Similarly, given a set $B \in \mathcal{B}_{r}^{*}$, let $P_{1}^{\epsilon}(B):=P(B) \cap\{x \in$ $\left.\mathbb{R}^{n}: \pi^{T} x \leq \pi_{0}^{1}+\epsilon\right\}$ and $P_{2}^{\epsilon}(B):=P(B) \cap\left\{x \in \mathbb{R}^{n}: \pi^{T} x \geq \pi_{0}^{2}-\epsilon\right\}$.

Now we prove $P_{1}^{\epsilon}$ and $P_{2}^{\epsilon}$ are full-dimensional by showing that $P_{1}^{\epsilon}$ and $P_{2}^{\epsilon}$ have interior points. Let $y_{1} \in P_{1} \neq \emptyset$, let $y_{2}$ be an interior point of $P$ and let $y(\lambda):=$ $\lambda y^{1}+(1-\lambda) y^{2}$, where $\lambda \in[0,1]$. Because $y_{2}$ is an interior point of $P, y(\lambda)$ is in the interior of $P$ for all $\lambda \in[0,1[$. Choose $\bar{\lambda} \in] 0,1[$ sufficiently close to one such that $\pi^{T} y(\bar{\lambda})<\pi_{0}^{1}+\epsilon$. We have $y(\bar{\lambda})$ is an interior point of $P_{1}^{\epsilon}$. This shows $P_{1}^{\epsilon}$ is a full-dimensional polyhedron. A similar proof applies to $P_{2}^{\epsilon}$. 
Since $P_{1}^{\epsilon}$ and $P_{2}^{\epsilon}$ are full-dimensional closed sets in $\mathbb{R}^{n}$, we have.

$$
\begin{aligned}
& \bigcap_{B \in \mathcal{B}_{r}^{*}} \operatorname{Conv}\left(P_{1}(B) \cup P_{2}(B)\right) \\
& =\bigcap_{B \in \mathcal{B}_{r}^{*}} \lim _{\epsilon \rightarrow 0^{+}} \operatorname{Conv}\left(P_{1}^{\epsilon}(B) \cup P_{2}^{\epsilon}(B)\right) \\
& =\lim _{\epsilon \rightarrow 0^{+}} \bigcap_{B \in \mathcal{B}_{r}^{*}} \operatorname{Conv}\left(P_{1}^{\epsilon}(B) \cup P_{2}^{\epsilon}(B)\right) \\
& =\lim _{\epsilon \rightarrow 0^{+}} \operatorname{Conv}\left(P_{1}^{\epsilon} \cup P_{2}^{\epsilon}\right) \\
& =\operatorname{Conv}\left(P_{1} \cup P_{2}\right) .
\end{aligned}
$$

In the remainder of this section assume $P \neq \emptyset$ and $P$ is not full-dimensional. Let $d_{P}:=\operatorname{dim}(A f f(P))<n$ denote the dimension of $P$ and let $M^{=}:=\left\{i \in M: a_{i}^{T} x=\right.$ $\left.b_{i}, \forall x \in P\right\}$ denote the constraints of $P$ satisfied with equality by all points in $P$. Since $\operatorname{dim}(P)=d_{P}$, there exists $B^{=} \in \mathcal{B}_{n-d_{P}}^{*}$ such that $\operatorname{Aff}(P)=\left\{x \in \mathbb{R}^{n}: a_{i .}^{T} x=b_{i}, i \in\right.$ $B^{=}$\}. Let $L$ denote the linear space parallel to $\operatorname{Aff}(P)$, i.e., $L:=\operatorname{Aff}(P)-x^{P}$, where $x^{P} \in P$.

The idea of the proof is to add a linear space $\bar{L}$ to $P$ in such a way that $P^{\prime}:=P+\bar{L}$ is full-dimensional. The space $\bar{L}$ is described in the following lemma.

Lemma 23. Let $L^{\pi}:=\left\{x \in \mathbb{R}^{n}: \pi^{T} x=0\right\}$. We may write

$$
L^{\pi}=\left(L^{\pi} \cap L\right)+\bar{L},
$$

where $\operatorname{dim}\left(L^{\pi} \cap L\right)=d_{P}-1, \operatorname{dim}(\bar{L})=n-d_{P}, L \cap \bar{L}=\left\{0_{n}\right\}$ and $L+\bar{L}=\mathbb{R}^{n}$.

Proof. Clearly $\operatorname{dim}\left(L^{\pi} \cap L\right)=d_{P}-1$ or $\operatorname{dim}\left(L^{\pi} \cap L\right)=d_{P}$. If $\operatorname{dim}\left(L^{\pi} \cap L\right)=d_{P}$ we have $\pi \in \operatorname{Span}\left(\left\{a_{i}\right\}_{i \in B^{=}}\right)$. This implies the function $\pi^{T} x$ is constant on $P$, which means that one (or both) of the sets $P_{1}$ and $P_{2}$ is empty. Since we have assumed this not to be the case, we have $\operatorname{dim}\left(L^{\pi} \cap L\right)=d_{P}-1$.

Let $\left\{b^{i}\right\}_{i=1}^{n-1}$ be a basis for $L^{\pi}$ chosen such that $\left\{b^{i}\right\}_{i=1}^{d_{P}-1} \subseteq L^{\pi} \cap L$. Define $\bar{L}:=$ $\operatorname{Span}\left(\left\{b^{i}\right\}_{i=d_{P}}^{n-1}\right)$. By construction $L^{\pi}=\left(L^{\pi} \cap L\right)+\bar{L}, \operatorname{dim}(\bar{L})=n-d_{P}$ and $L \cap \bar{L}=\left\{0_{n}\right\}$.

To prove $L+\bar{L}=\mathbb{R}^{n}$, observe that $L^{\pi}=\left(L^{\pi} \cap L\right)+\bar{L} \subseteq L+\bar{L}$. We cannot have $x \in L^{\pi}$ for all $x \in L$, since that would imply that $\pi^{T} x$ is constant on $P$.

Observe that the fact $L+\bar{L}=\mathbb{R}^{n}$ implies that $P^{\prime}:=P+\bar{L}$ is full-dimensional (we have $\left.\operatorname{Aff}\left(P^{\prime}\right)=\operatorname{Aff}(P)+\bar{L}=x^{P}+L+\bar{L}=\mathbb{R}^{n}\right)$. Also observe that the set $\bar{L}$ has been constructed as a subspace of $L^{\pi}$ and therefore that $\pi^{T} \bar{l}=0$ for all $\bar{l} \in \bar{L}$. We now relate the sets $P$ and $P^{\prime}$ and the sets $\operatorname{Conv}\left(P \cap F_{\left(\pi, \pi_{0}^{1}, \pi_{0}^{2}\right)}\right)$ and $\operatorname{Conv}\left(P^{\prime} \cap F_{\left(\pi, \pi_{0}^{1}, \pi_{0}^{2}\right)}\right)$.

Lemma 24. (i) $P^{\prime} \cap \operatorname{Aff}(P)=P$.

(ii) $\operatorname{Conv}\left(P^{\prime} \cap F_{\left(\pi, \pi_{0}^{1}, \pi_{0}^{2}\right)}\right)=\operatorname{Conv}\left(P \cap F_{\left(\pi, \pi_{0}^{1}, \pi_{0}^{2}\right)}\right)+\bar{L}$.

Proof. (ii) follows easily from the facts that $P^{\prime}=P+\bar{L}$ and $\pi^{T} \bar{l}=0$ for all $\bar{l} \in \bar{L}$, so we only prove (i). Since $P \subseteq P^{\prime}$ and $P \subseteq \operatorname{Aff}(P)$, we have $P \subseteq P^{\prime} \cap \operatorname{Aff}(P)$. Let 
$\bar{y} \in P^{\prime} \cap A f f(P)$. Since $\bar{y} \in P^{\prime}$, we have $\bar{y}=z+\bar{l}$, where $z \in P$ and $\bar{l} \in \bar{L}$. Also, since $\bar{y} \in \operatorname{Aff}(P)$ we have $\bar{y}=x^{P}+l$, where $l \in L$. This implies $\left(\bar{y}-x^{P}\right)=\left(z-x^{P}\right)+\bar{l}$. Since $\left(\bar{y}-x^{P}\right),\left(z-x^{P}\right) \in L$, we have $\left(\bar{y}-x^{P}\right)-\left(z-x^{P}\right) \in L \cap \bar{L}$. Hence $\left(\bar{y}-x^{P}\right)-\left(z-x^{P}\right)=0_{n}$, or, $\bar{y}=z \in P$, which proves that $P^{\prime} \cap A f f(P) \subseteq P$.

We now give a description of $P^{\prime}$ with constraints. The description is based on the constraints of $P$, and an affine mapping $\tilde{A}_{f}: \mathbb{R}^{n} \mapsto \mathbb{R}^{n}$ with the following properties.

Lemma 25. There exist $\tilde{D} \in \mathbb{R}^{n \times n}$ and $\tilde{d} \in \mathbb{R}^{n}$ such that the affine mapping $\tilde{A}_{f}(x):=$ $\tilde{D} x+\tilde{d}$ and the corresponding linear mapping $\tilde{L}_{f}(x):=\tilde{D} x$ satisfy

(i) $\tilde{A}_{f}(x)=0_{n}$ for all $x \in A f f(P)$, i.e., $\tilde{A}_{f}$ maps $A f f(P)$ to the set $\left\{0_{n}\right\}$.

(ii) $\tilde{L}_{f}(x)=0_{n}$ for all $x \in L$, i.e., $\tilde{L}_{f}$ maps $L$ to the set $\left\{0_{n}\right\}$.

(iii) For $x, \bar{l} \in \mathbb{R}^{n}, \bar{l}=\tilde{A}_{f}(x) \Longleftrightarrow \bar{l} \in \bar{L}$ and $(x-\bar{l}) \in \operatorname{Aff}(P)$.

(iv) For $x, \bar{l} \in \mathbb{R}^{n}, \bar{l}=\tilde{L}_{f}(x) \Longleftrightarrow \bar{l} \in \bar{L}$ and $(x-\bar{l}) \in L$.

Proof. Since $\bar{L}$ is a vector space, there exist linearly independent vectors $\left\{\bar{b}^{k}\right\}_{k \in I}$, where $I:=\left\{1,2, \ldots, d_{P}\right\}$, such that $\bar{L}=\left\{\bar{l} \in \mathbb{R}^{n}:\left(\bar{b}^{k}\right)^{T} \bar{l}=0, \forall k \in I\right\}$. Notice that the vectors $\left\{\bar{b}^{k}\right\}_{k \in I}$ define a basis for $\bar{L}^{\perp}$, and that the vectors $\left\{a_{i}\right\}_{i \in B}=$ define a basis for $L^{\perp}$. Since $L+\bar{L}=\mathbb{R}^{n}$ and $L \cap \bar{L}=\left\{0_{n}\right\}$, it follows that $L^{\perp}+\bar{L}^{\perp}=\mathbb{R}^{n}$, so that the vectors $\left\{\bar{b}^{k}\right\}_{k \in I} \cup\left\{a_{i .}\right\}_{i \in B}=$ are linearly independent. Now we have:

$$
\begin{aligned}
& \bar{l} \in \bar{L} \text { and }(x-\bar{l}) \in \operatorname{Aff}(P) \Longleftrightarrow \\
& \bar{l} \in \bar{L} \text { and } a_{i .}^{T}(x-\bar{l})=b_{i}, \forall i \in B^{=} \Longleftrightarrow \\
& a_{i .}^{T}(x-\bar{l})=b_{i}, \forall i \in B^{=} \text {and }\left(\bar{b}^{k}\right)^{T} \bar{l}=0, \forall k \in I \Longleftrightarrow \\
& \bar{l}=\tilde{D} x+\tilde{d},
\end{aligned}
$$

where $\tilde{D}$ is the inverse matrix of $\left[a_{i} \mid \bar{b}^{k}\right]^{T}$. Observe that (28) expresses $\bar{l}$ as a linear function of $x$. Next it is an easy exercise to verify the correctness of (i)-(iv).

We now have the following description of $P^{\prime}$ with constraints

Lemma 26. Let $\tilde{A}_{f}$ be as in Lemma 25. Then

$$
P^{\prime}=\left\{x \in \mathbb{R}^{n}: a_{i .}^{T} x \leq b_{i}+a_{i .}^{T} \tilde{A}_{f}(x), \forall i \in M \backslash M^{=}\right\} .
$$

Proof. We have $x \in P^{\prime} \Longleftrightarrow \exists \bar{l} \in \bar{L}$ such that $a_{i}^{T}(x-\bar{l}) \leq b_{i}, \forall i \in M \backslash M^{=}$, and $a_{i .}^{T}(x-\bar{l})=b_{i}, \forall i \in B^{=} \Longleftrightarrow$ (by Lemma 25(iii)) $\exists \bar{l} \in \bar{L}$ such that $a_{i .}^{T}(x-\bar{l}) \leq$ $b_{i}, \forall i \in M \backslash M^{=}$, and $\bar{l}=\tilde{A}_{f}(x) \Longleftrightarrow a_{i .}^{T} x \leq b_{i}+a_{i .}^{T} \tilde{A}_{f}(x), \forall i \in M \backslash M^{=}$.

Lemma 26 shows that $P^{\prime}$ has one constraint for each element of $M \backslash M^{=}$. Let $r^{\prime}$ denote the rank of the corresponding coefficient matrix, i.e., $r^{\prime}$ is the size of a maximal subset $B^{\prime}$ of $M \backslash M^{=}$such that the vectors $\left\{a_{i}^{T}\left(I_{n}-\tilde{D}\right)\right\}_{i \in B^{\prime}}$ are linearly independent ( $I_{n}$ denotes the $n$-by- $n$ identity matrix). Furthermore let $\mathcal{B}_{r^{\prime}}^{\prime *}$ denote the set of all such subsets.

It is easy to see that, if the vectors $\left\{a_{i}^{T}\left(I_{n}-\tilde{D}\right)\right\}_{i \in B^{\prime}}$ are linearly independent, then so are the vectors $\left\{a_{i .}\right\}_{i \in B^{\prime}}$. Specifically, if the vectors $\left\{a_{i .}\right\}_{i \in B^{\prime}}$ are linearly dependent, then one of them can be expressed as a linear combination of the rest. However, that would 
mean that the corresponding vector in $\left\{a_{i .}^{T}\left(I_{n}-\tilde{D}\right)\right\}_{i \in B^{\prime}}$ is a linear combination of the remaining vectors in $\left\{a_{i}^{T}\left(I_{n}-\tilde{D}\right)\right\}_{i \in B^{\prime}}$, which contradicts their linear independence.

We know that $P$ is not full-dimensional, but that $P^{\prime}$ is full-dimensional, where $P^{\prime}:=P+\bar{L}$ and $\bar{L}$ is the linear space described by Lemma 23. Similarly, $P\left(B^{\prime}\right) \cap A f f(P)$ is not full-dimensional for any $B^{\prime} \in \mathcal{B}^{\prime *}{ }_{r^{\prime}}$, but we can also add $\bar{L}$ to it to get a full-dimensional conic polyhedron. Given a set $B^{\prime} \in \mathcal{B}_{r^{\prime}}{ }^{\prime}$, define $P^{\prime}\left(B^{\prime}\right):=\left\{x \in \mathbb{R}^{n}: a_{i .}^{T} x \leq\right.$ $\left.b_{i}+a_{i .}^{T} \tilde{A}_{f}(x), \forall i \in B^{\prime}\right\} . P^{\prime}\left(B^{\prime}\right)$ denotes the conic polyhedron for $P^{\prime}$ associated with $B^{\prime}$. We now relate the set $P^{\prime}\left(B^{\prime}\right)$ to $P\left(B^{\prime}\right)$ for $B^{\prime} \in \mathcal{B}_{r^{\prime}}^{\prime *}$.

Lemma 27. Let $B^{\prime} \in \mathcal{B}_{r^{\prime}}^{*}$. Then

$$
P^{\prime}\left(B^{\prime}\right)=P\left(B^{\prime}\right) \cap A f f(P)+\bar{L}
$$

Proof. Using Lemma 25(iii) we get $x \in P^{\prime}\left(B^{\prime}\right) \Longleftrightarrow a_{i .}^{T}(x-\bar{l}) \leq b_{i}, \forall i \in B^{\prime}$, $a_{i}^{T}(x-\bar{l})=b_{i}, \forall i \in B^{=}, \bar{l} \in \bar{L}$ and $\bar{l}=\tilde{A}_{f}(x) \Longleftrightarrow x=y+\bar{l}$, where $y \in$ $P\left(B^{\prime}\right) \cap A f f(P), \bar{l} \in \bar{L}$ and $\bar{l}=\tilde{A}_{f}(x) \Longleftrightarrow x \in P\left(B^{\prime}\right) \cap A f f(P)+\bar{L}$.

Next we show that it is possible to find a representation of the conic polyhedron $P^{\prime}\left(B^{\prime}\right)$ such that the extreme point $\bar{x}$ is in $\operatorname{Aff}(P)$, and the extreme rays $\left\{r^{k}\right\}_{k \in B^{\prime}}$ are all in $L$.

Lemma 28. Let $B^{\prime} \in \mathcal{B}_{r^{\prime}}^{*}$. There exist vectors $\bar{x} \in A f f(P)$ and $\left\{r^{k}\right\}_{k \in B^{\prime}} \subseteq L$ such that

$$
P^{\prime}\left(B^{\prime}\right)=\bar{x}+L^{\prime}\left(B^{\prime}\right)+\text { Cone }\left(\left\{r^{k}\right\}_{k \in B^{\prime}}\right),
$$

where $L^{\prime}\left(B^{\prime}\right):=\left\{x \in \mathbb{R}^{n}: a_{i .}^{T} x=a_{i .}^{T} \tilde{L}_{f}(x), \forall i \in B^{\prime}\right\}$, i.e., $L^{\prime}\left(B^{\prime}\right):=\left\{x \in \mathbb{R}^{n}\right.$ : $\left.a_{i .}^{T}(x-\tilde{D} x)=0, \forall i \in B^{\prime}\right\}$. The vector $\bar{x}$ satisfies $a_{i .}^{T} \bar{x}=b_{i}$ for all $i \in B^{\prime} \cup B^{=}$. Furthermore given $k \in B^{\prime}$, the vector $r^{k}$ satisfies

(i) $a_{i}^{T} r^{k}=0, \forall i \in\left(B^{\prime} \cup B^{=}\right) \backslash\{k\}$ and

(ii) $a_{k}^{\dot{T}} r^{k}=-1$.

Proof. Since $P^{\prime}\left(B^{\prime}\right)$ is a conic polyhedron we may write (see Sect. 2.1)

$$
P^{\prime}\left(B^{\prime}\right)=\bar{y}+L^{\prime}\left(B^{\prime}\right)+\text { Cone }\left(\left\{s^{k}\right\}_{k \in B^{\prime}}\right)
$$

where the vectors $\bar{y}$ and $\left\{s^{k}\right\}_{k \in B^{\prime}}$ are as follows. The vector $\bar{y}$ satisfies $a_{i}^{T} \bar{y}=b_{i}+$ $a_{i .}^{T} \tilde{A}_{f}(\bar{y})$ for all $i \in B^{\prime}$. Given $k \in B^{\prime}$, the vector $s^{k}$ satisfies $a_{i .}^{T} s^{k}=a_{i .}^{T} \tilde{L}_{f}\left(s^{k}\right), \forall i \in$ $B^{\prime} \backslash\{k\}$ and $a_{k}^{T} s^{k}=a_{k}^{T} \tilde{L}_{f}\left(s^{k}\right)-1$.

Define $\bar{l}:=\tilde{A}_{f}(\bar{y})$, and define the vector $\bar{x}:=\bar{y}-\bar{l}$. From Lemma 25(iii) we have $\bar{x} \in \operatorname{Aff}(P)$ and $\bar{l} \in \bar{L}$. Since $\bar{x} \in \operatorname{Aff}(P)$ Lemma 25(i) implies $\tilde{A}_{f}(\bar{x})=0_{n}$. Furthermore, for $i \in B^{\prime}$, we have $a_{i .}^{T} \bar{x}=a_{i .}^{T} \bar{y}-a_{i .}^{T} \bar{l}=b_{i}+a_{i .}^{T} \tilde{A}_{f}(\bar{y})-a_{i .}^{T} \bar{l}=b_{i}$. Also, since $\bar{x} \in \operatorname{Aff}(P)$, we have $a_{i .}^{T} \bar{x}=b_{i}$ for all $i \in B^{=}$. Finally, since $\tilde{A}_{f}(\bar{x})=0_{n}$, we have $a_{i .}^{T} \bar{x}=b_{i}+a_{i .}^{T} \tilde{A}_{f}(\bar{x}), \forall i \in B^{\prime}$. Hence we can use $\bar{x}$ as an extreme point for $P^{\prime}\left(B^{\prime}\right)$, $\bar{x} \in \operatorname{Aff}(P)$ and $a_{i}^{T} \bar{x}=b_{i}$ for all $i \in B^{\prime} \cup B^{=}$.

We now define the vectors $r^{k}$ for $k \in B^{\prime}$. Let $k \in B^{\prime}$ be arbitrary, and consider the vector $s^{k}$ defined above. Let $\bar{l}:=\tilde{L}_{f}\left(s^{k}\right)$ and define $r^{k}$ as $r^{k}:=s^{k}-\bar{l}$. From Lemma 25(iv) we have $r^{k} \in L$. Moreover, since $r^{k} \in L$, Lemma 25(ii) implies $\tilde{L}_{f}\left(r^{k}\right)=0_{n}$. 
Also, for $i \in B^{\prime} \backslash\{k\}$, we have $a_{i .}^{T} r^{k}=a_{i .}^{T} s^{k}-a_{i .}^{T} \bar{l}=a_{i .}^{T}\left(\tilde{L}_{f}\left(s^{k}\right)-\bar{l}\right)=0$, and $a_{k}^{T} r^{k}=$ $a_{k .}^{T} s^{k}-a_{k .}^{T} \bar{l}=a_{k}^{T}\left(\tilde{L}_{f}\left(s^{k}\right)-\bar{l}\right)-1=-1$. Since $r^{k} \in L$ we have $a_{i .}^{T} r^{k}=0, \forall i \in B=$. Finally, since $\tilde{L}_{f}\left(r^{k}\right)=0_{n}, a_{i .}^{T} r^{k}=\tilde{L}_{f}\left(r^{k}\right), \forall i \in B^{\prime} \backslash\{k\}$ and $a_{k}^{T} r^{k}=a_{k}^{T} \tilde{L}_{f}\left(r^{k}\right)-1$. Hence we can use $r^{k}$ as an extreme ray for $P^{\prime}\left(B^{\prime}\right), r^{k} \in L$ and $r^{k}$ satisfies (i) and (ii).

The choice of extreme point and extreme rays in Lemma 28 implies the following facts about the set $P\left(B^{\prime} \cup B\right)$ and the conic polyhedron $P\left(B^{\prime}\right)$.

Lemma 29. Let $B^{\prime} \in \mathcal{B}_{r^{\prime}}^{*}$ and let the vectors $\bar{x} \in \operatorname{Aff}(P)$ and $\left\{r^{k}\right\}_{k \in B^{\prime}} \subseteq L$ be as in Lemma 28.

(i) The vectors $\left\{a_{i}\right\}_{i \in B^{\prime} \cup B}$ are linearly independent, i.e., $P\left(B^{\prime} \cup B\right)$ is a conic polyhedron.

(ii) $P\left(B^{\prime}\right)=\bar{x}+L\left(B^{\prime}\right)+$ Cone $\left(\left\{r^{k}\right\}_{k \in B^{\prime}}\right)$, where $L\left(B^{\prime}\right)$ is given by $L\left(B^{\prime}\right)=\{x \in$ $\left.\mathbb{R}^{n}: a_{i .}^{T} x=0, \forall i \in B^{\prime}\right\}$.

(iii) $P\left(B^{\prime}\right) \cap \operatorname{Aff}(P)=\bar{x}+L\left(B^{\prime} \cup B^{=}\right)+$Cone $\left(\left\{r^{k}\right\}_{k \in B^{\prime}}\right)$, where $L\left(B^{\prime} \cup B^{=}\right)=$ $L \cap L\left(B^{\prime}\right)$.

Proof. (ii) and (iii) follow from the properties of the vectors $\bar{x}$ and $\left\{r^{k}\right\}_{k \in B^{\prime}}$, so we only prove (i). Let $k \in B^{\prime} \cup B^{=}$, and assume $a_{k}=\sum_{i \in\left(B^{\prime} \cup B^{=}\right) \backslash\{k\}} \mu_{i} a_{i}$, where $\mu_{i} \in \mathbb{R}$ for $i \in\left(B^{\prime} \cup B^{=}\right) \backslash\{k\}$. From multiplying this equality by $\bar{x}$ and using the fact that $a_{i}^{T} \bar{x}=b_{i}$ for all $\mathrm{i} \in B^{\prime} \cup B^{=}$, we have $b_{k}=\sum_{i \in\left(B^{\prime} \cup B^{=}\right) \backslash\{k\}} \mu_{i} b_{i}$. For any $\bar{i} \in B^{\prime} \backslash\{k\}$ we have $0=a_{k}^{T} r^{\bar{i}}=\sum_{i \in\left(B^{\prime} \cup B^{=}\right) \backslash\{k\}} \mu_{i} a_{i .}^{T} r^{\bar{i}}=-\mu_{\bar{i}}$. Hence $\mu_{i}=0$ for all $i \in B^{\prime} \backslash\{k\}$. Assume by contradiction that $k \in B^{\prime}$. Then $a_{k}$. $=\sum_{i \in B=\mu_{i}} a_{i}$. and $b_{k}=\sum_{i \in B}=\mu_{i} b_{i}$. This implies $k \in M^{=}$, which contradicts $B^{\prime} \subseteq M \backslash M^{=}$. Therefore we can assume $k \in B^{=}$. The equality $a_{k}=\sum_{i \in B^{=} \backslash\{k\}} \mu_{i} a_{i}$. contradicts the fact that $B^{=} \in \mathcal{B}_{n-d_{P}}^{*}\left(B^{=}\right.$is defined before Lemma 23).

Lemma 29(ii)-(iii) gives that both sets $P\left(B^{\prime}\right)$ and $P\left(B^{\prime}\right) \cap A f f(P)$ can be generated from the vectors $\bar{x}$ and $\left\{r^{k}\right\}_{k \in B^{\prime}}$ of Lemma 28. The following lemma describes the disjunctive hull associated with a set $B^{\prime} \in \mathcal{B}_{r^{\prime}}^{*}$ and $D\left(\pi, \pi_{0}^{1}, \pi_{0}^{2}\right)$.

Lemma 30. Let $B^{\prime} \in \mathcal{B}_{r^{\prime}}^{*}$.

(i) $\left.\pi^{T} x \in\right] \pi_{0}^{1}, \pi_{0}^{2}\left[, \forall x \in \bar{x}+L^{\prime}\left(B^{\prime}\right) \Longleftrightarrow \pi^{T} x \in\right] \pi_{0}^{1}, \pi_{0}^{2}\left[, \forall x \in \bar{x}+L\left(B^{\prime} \cup B^{=}\right)\right.$, i.e., an intersection cut can be derived from $P\left(B^{\prime} \cup B^{=}\right)$if and only if an intersection cut can be derived from $P^{\prime}\left(B^{\prime}\right)$.

(ii) $\operatorname{Conv}\left(P^{\prime}\left(B^{\prime}\right) \cap F_{D\left(\pi, \pi_{0}^{1}, \pi_{0}^{2}\right)}\right)=\operatorname{Conv}\left(P\left(B^{\prime} \cup B^{=}\right) \cap F_{D\left(\pi, \pi_{0}^{1}, \pi_{0}^{2}\right)}\right) \cap A f f(P)+\bar{L}$.

Proof. (i) We first prove that $\bar{x}+L\left(B^{\prime} \cup B^{=}\right) \subseteq \bar{x}+L^{\prime}\left(B^{\prime}\right)$

$$
\begin{array}{rlr}
y \in \bar{x}+L\left(B^{\prime} \cup B^{=}\right) & \Rightarrow & \text { (Lemma 28) } \\
a_{i .}^{T} y=b_{i}, \forall i \in B^{=} \cup B^{\prime} & \Rightarrow & \\
y \in A f f(P) \text { and } a_{i .}^{T} y=b_{i}, \forall i \in B^{\prime} & \Rightarrow & (\operatorname{Lemma} 25(i)) \\
\tilde{A}_{f}(y)=0_{n} \text { and } a_{i .}^{T} y=b_{i}+a_{i .}^{T} \tilde{A}_{f}(y), \forall i \in B^{\prime} & \Rightarrow & \\
y \in \bar{x}+L^{\prime}\left(B^{\prime}\right) &
\end{array}
$$

This shows $\left.\pi^{T} x \in\right] \pi_{0}^{1}, \pi_{0}^{2}\left[, \forall x \in \bar{x}+L^{\prime}\left(B^{\prime}\right) \Rightarrow \pi^{T} x \in\right] \pi_{0}^{1}, \pi_{0}^{2}\left[, \forall x \in \bar{x}+L\left(B^{\prime} \cup B^{=}\right)\right.$. 
For the other direction, suppose $y \in \bar{x}+L^{\prime}\left(B^{\prime}\right)$ and $\left.\pi^{T} y \notin\right] \pi_{0}^{1}, \pi_{0}^{2}[$. We will show that this implies there exists $z \in \bar{x}+L\left(B^{\prime} \cup B^{=}\right)$satisfying $\left.\pi^{T} z \notin\right] \pi_{0}^{1}, \pi_{0}^{2}[$. Define $\bar{l}:=\tilde{A}_{f}(y)$. From Lemma 25(iii), we have $\bar{l} \in \bar{L}$ and $z:=y-\bar{l} \in A f f(P)$. This implies $a_{i}^{T} z=b_{i}, \forall i \in B^{=} \cup B^{\prime}$, i.e., $z \in \bar{x}+L\left(B^{\prime} \cup B^{=}\right)$. Since $\pi^{T} \bar{l}=0$ (Lemma 23), we have $\left.\pi^{T} z=\pi^{T}(y-\bar{l})=\pi^{T} y \notin\right] \pi_{0}^{1}, \pi_{0}^{2}$ [. This finishes the proof of (i).

(ii) We may assume $\left.\pi^{T} y \in\right] \pi_{0}^{1}, \pi_{0}^{2}$ [ for all $y \in \bar{x}+L^{\prime}\left(B^{\prime}\right)$, since otherwise statement (ii) reduces to $P^{\prime}\left(B^{\prime}\right)=P\left(B^{\prime}\right) \cap A f f(P)+\bar{L}$ (Lemma 27$)$.

From Lemma 28 and 29 it follows that the intersection cut associated with $P\left(B^{\prime} \cup B^{=}\right)$ and $D\left(\pi, \pi_{0}^{1}, \pi_{0}^{2}\right)$ is given by

$$
\sum_{k \in B^{\prime}}\left(b_{k}-a_{k}^{T} x\right) / \alpha_{k} \geq 1
$$

for points $x \in A f f(P)$, where

$$
\alpha_{k}:= \begin{cases}-\epsilon^{1} /\left(\pi^{T} r^{k}\right) & \text { if } \pi^{T} r^{k}<0, \\ \epsilon^{2} /\left(\pi^{T} r^{k}\right) & \text { if } \pi^{T} r^{k}>0, \\ +\infty & \text { otherwise }\end{cases}
$$

for $k \in B^{\prime}, \epsilon^{1}:=\pi^{T} \bar{x}-\pi_{0}^{1}$ and $\epsilon^{2}:=\pi_{0}^{2}-\pi^{T} \bar{x}$ (see the definition in (2)). Similarly the intersection cut associated with $P^{\prime}\left(B^{\prime}\right)$ and $D\left(\pi, \pi_{0}^{1}, \pi_{0}^{2}\right)$ is given by

$$
\sum_{k \in B^{\prime}}\left(b_{k}+a_{k .}^{T} \tilde{A}_{f}(x)-a_{k .}^{T} x\right) / \alpha_{k} \geq 1 .
$$

Now suppose $x \in \operatorname{Conv}\left(P^{\prime}\left(B^{\prime}\right) \cap F_{D\left(\pi, \pi_{0}^{1}, \pi_{0}^{2}\right)}\right)$. Since $x \in P^{\prime}\left(B^{\prime}\right)$, Lemma 27 shows that we may write $x=y+\bar{l}$, where $y \in P\left(B^{\prime}\right) \cap A f f(P), \bar{l}:=\tilde{A}_{f}(x)$ and $\bar{l} \in \bar{L}$. From the fact that $x$ satisfies the intersection cut (31) associated with $P^{\prime}\left(B^{\prime}\right)$, we have $\sum_{k \in B^{\prime}}\left(b_{k}+a_{k .}^{T} \tilde{A}_{f}(x)-a_{k .}^{T} x\right) / \alpha_{k} \geq 1$. Because $\bar{l}=\tilde{A}_{f}(x), x$ satisfies $\sum_{k \in B^{\prime}}\left(b_{k}+\right.$ $\left.a_{k}^{T} \bar{l}-a_{k}^{T} x\right) / \alpha_{k} \geq 1$, it follows that $\sum_{k \in B^{\prime}}\left(b_{k}-a_{k .}^{T} y\right) / \alpha_{k} \geq 1$, i.e., $y$ satisfies the intersection cut (29) associated with $P\left(B^{\prime} \cap B^{=}\right)$.

Finally suppose $x \in \operatorname{Conv}\left(P\left(B^{\prime} \cup B^{=}\right) \cap F_{D\left(\pi, \pi_{0}^{1}, \pi_{0}^{2}\right)}\right) \cap A f f(P)+\bar{L}$. Write $x=y+\bar{l}$, where $y \in \operatorname{Conv}\left(P\left(B^{\prime} \cup B^{=}\right) \cap F_{D\left(\pi, \pi_{0}^{1}, \pi_{0}^{2}\right)}\right) \cap \operatorname{Aff}(P)$ and $\bar{l} \in \bar{L}$. Since $y=x-\bar{l} \in \operatorname{Conv}\left(P\left(B^{\prime} \cup B^{=}\right) \cap F_{D\left(\pi, \pi_{0}^{1}, \pi_{0}^{2}\right)}\right) \cap A f f(P)$ and $\bar{l} \in \bar{L}$, we have $\bar{l}=\tilde{A}_{f}(x)$. From the fact that $y$ satisfies the intersection cut (29) associated with $P\left(B^{\prime} \cup B^{=}\right)$and $D\left(\pi, \pi_{0}^{1}, \pi_{0}^{2}\right)$ it follows that $x$ satisfies the intersection cut (31) associated with $P^{\prime}\left(B^{\prime}\right)$ and $D\left(\pi, \pi_{0}^{1}, \pi_{0}^{2}\right)$.

We are now able to finish the proof of Theorem 1.

Lemma 31. Theorem 1 holds when $P, P_{1}$ and $P_{2}$ are non-empty and not full-dimensional.

Proof. Let $B^{\prime} \in \mathcal{B}_{r^{\prime}}^{\prime *}$ be arbitrary. We first prove that $\operatorname{Conv}\left(P(\bar{B}) \cap F_{D\left(\pi, \pi_{0}^{1}, \pi_{0}^{2}\right)}\right) \subseteq$ $\operatorname{Conv}\left(P\left(B^{\prime} \cup B^{=}\right) \cap F_{D\left(\pi, \pi_{0}^{1}, \pi_{0}^{2}\right)}\right)$. We distinguish two cases: 
(a) If $\left.\pi^{T} y \in\right] \pi_{0}^{1}, \pi_{0}^{2}$ [ for all $y \in \bar{x}+L\left(B^{\prime} \cup B^{=}\right)$:

An intersection cut $\bar{\delta}^{T} x \geq \bar{\delta}_{0}$ can be derived from $B^{\prime} \cup B^{=}$and $D\left(\pi, \pi_{0}^{1}, \pi_{0}^{2}\right)$. The inequality $\bar{\delta}^{T} x \geq \bar{\delta}_{0}$ is also an intersection cut for $\bar{B}$ and $D\left(\pi, \pi_{0}^{1}, \pi_{0}^{2}\right)$ for any $\bar{B} \in \mathcal{B}_{r}^{*}$ satisfying $\bar{B} \supseteq B^{\prime} \cup B^{=}$(Lemma 10). Furthermore we have $\operatorname{Conv}(P(\bar{B}) \cap$ $F_{D\left(\pi, \pi_{0}^{1}, \pi_{0}^{2}\right)} \subseteq \operatorname{Conv}\left(P\left(B^{\prime} \cup B^{=}\right) \cap F_{D\left(\pi, \pi_{0}^{1}, \pi_{0}^{2}\right)}\right)$.

(b) If $\left.\pi^{T} y \in\right] \pi_{0}^{1}, \pi_{0}^{2}\left[\right.$ for some $y \in \bar{x}+L\left(B^{\prime} \cup B^{=}\right)$:

$\operatorname{Conv}\left(P\left(B^{\prime} \cup B^{=}\right) \cap F_{D\left(\pi, \pi_{0}^{1}, \pi_{0}^{2}\right)}\right)=P\left(B^{\prime} \cup B^{=}\right)$. Also $P\left(B^{\prime} \cup B^{=}\right) \supseteq P(\bar{B}) \supseteq$ $\operatorname{Conv}\left(P(\bar{B}) \cap F_{D\left(\pi, \pi_{0}^{1}, \pi_{0}^{2}\right)}\right)$ for all $\bar{B} \in \mathcal{B}_{r}^{*}$ satisfying $\bar{B} \supseteq B^{\prime} \cup B^{=}$.

Therefore for any $B^{\prime} \in \mathcal{B}_{r^{\prime}}^{\prime *}$ there exists $\bar{B} \in \mathcal{B}_{r}^{*}$ s.t. $\operatorname{Conv}\left(P(\bar{B}) \cap F_{D\left(\pi, \pi_{0}^{1}, \pi_{0}^{2}\right)}\right) \subseteq$ $\operatorname{Conv}\left(P\left(B^{\prime} \cup B^{=}\right) \cap F_{D\left(\pi, \pi_{0}^{1}, \pi_{0}^{2}\right)}\right)$. Hence we can choose $\overline{\mathcal{B}}_{r}^{*} \subseteq \mathcal{B}_{r}^{*}$ such that $\cap_{B^{\prime} \in \mathcal{B}^{\prime *}{ }^{\prime}} \operatorname{Conv}\left(P\left(B^{\prime} \cup B^{=}\right) \cap F_{D\left(\pi, \pi_{0}^{1}, \pi_{0}^{2}\right)}\right) \supseteq \cap_{\bar{B} \in \overline{\mathcal{B}}_{r}^{*}} \operatorname{Conv}\left(P(\bar{B}) \cap F_{D\left(\pi, \pi_{0}^{1}, \pi_{0}^{2}\right)}\right)$.

Since $\overline{\mathcal{B}}_{r}^{*}$ is a subset of $\mathcal{B}_{r}^{*}$, we have the inclusion

$$
\cap_{B^{\prime} \in \mathcal{B}_{r^{\prime}}^{\prime *}} \operatorname{Conv}\left(P\left(B^{\prime} \cup B^{=}\right) \cap F_{D\left(\pi, \pi_{0}^{1}, \pi_{0}^{2}\right)}\right) \supseteq \cap_{B \in \mathcal{B}_{r}^{*}} \operatorname{Conv}\left(P(B) \cap F_{D\left(\pi, \pi_{0}^{1}, \pi_{0}^{2}\right)}\right) \text {. }
$$

Using the fact that $P^{\prime}$ is full-dimensional, Lemma 22 gives the identity $\operatorname{Conv}\left(P^{\prime} \cap F_{D\left(\pi, \pi_{0}^{1}, \pi_{0}^{2}\right)}\right)=\cap_{B^{\prime} \in \mathcal{B}^{\prime}{ }_{r^{\prime}}} \operatorname{Conv}\left(P^{\prime}\left(B^{\prime}\right) \cap F_{D\left(\pi, \pi_{0}^{1}, \pi_{0}^{2}\right)}\right)$. Also Lemma 24(ii) states $\operatorname{Conv}\left(P^{\prime} \cap F_{D\left(\pi, \pi_{0}^{1}, \pi_{0}^{2}\right)}\right)=\operatorname{Conv}\left(P \cap F_{D\left(\pi, \pi_{0}^{1}, \pi_{0}^{2}\right)}\right)+\bar{L}$. This implies that $\operatorname{Conv}(P \cap$ $\left.F_{D\left(\pi, \pi_{0}^{1}, \pi_{0}^{2}\right)}\right)+\bar{L}=\cap_{B^{\prime} \in \mathcal{B}^{\prime *}{ }^{\prime}} \operatorname{Conv}\left(P^{\prime}\left(B^{\prime}\right) \cap F_{D\left(\pi, \pi_{0}^{1}, \pi_{0}^{2}\right)}\right)$. Using Lemma 30(ii) gives that $\bar{L}+\operatorname{Aff}(P) \cap\left(\cap_{B^{\prime} \in \mathcal{B}^{\prime}{ }_{r^{\prime}}} \operatorname{Conv}\left(P\left(B^{\prime} \cup B^{=}\right) \cap F_{D\left(\pi, \pi_{0}^{1}, \pi_{0}^{2}\right)}\right)\right)=\bar{L}+\operatorname{Conv}(P \cap$ $\left.F_{D\left(\pi, \pi_{0}^{1}, \pi_{0}^{2}\right)}\right)$. Since $\cap_{B^{\prime} \in \mathcal{B}_{r^{\prime}}^{*}} \operatorname{Conv}\left(P\left(B^{\prime} \cup B^{=}\right) \cap F_{D\left(\pi, \pi_{0}^{1}, \pi_{0}^{2}\right)}\right) \supseteq \cap_{B \in \mathcal{B}_{r}^{*}} \operatorname{Conv}(P(B)$ $\left.\cap F_{D\left(\pi, \pi_{0}^{1}, \pi_{0}^{2}\right)}\right)$ and $\cap_{B \in \mathcal{B}_{r}^{*}} \operatorname{Conv}\left(P(B) \cap F_{D\left(\pi, \pi_{0}^{1}, \pi_{0}^{2}\right)} \subseteq P \subseteq \operatorname{Aff}(P)\right.$ we have $\bar{L}+$ $\operatorname{Conv}\left(P \cap F_{D\left(\pi, \pi_{0}^{1}, \pi_{0}^{2}\right)}\right) \supseteq \bar{L}+\cap_{B \in \mathcal{B}_{r}^{*}} \operatorname{Conv}\left(P(B) \cap F_{D\left(\pi, \pi_{0}^{1}, \pi_{0}^{2}\right)}\right)$. This implies $\operatorname{Conv}(P \cap$ $F_{D\left(\pi, \pi_{0}^{1}, \pi_{0}^{2}\right)} \supseteq \cap_{B \in \mathcal{B}_{r}^{*}} \operatorname{Conv}\left(P(B) \cap F_{D\left(\pi, \pi_{0}^{1}, \pi_{0}^{2}\right)}\right)$ (by intersecting with $\operatorname{Aff}(P)$ on both sides). Lemma 11 shows the other direction.

\section{Disjunctive hulls derived from polyhedra and two-term disjunctions}

We now consider two-term disjunctions $D$ of the form $D^{1} x \leq d^{1} \vee D^{2} x \leq d^{2}$, where $D^{1} \in \mathbb{R}^{m_{1} \times n}, D^{2} \in \mathbb{R}^{m_{2} \times n}, d^{1} \in \mathbb{R}^{m_{1}}$ and $d^{2} \in \mathbb{R}^{m_{2}}$. The set of points $\bar{x} \in \mathbb{R}^{n}$ that satisfy $D$ is denoted $F_{D}$. In this section, we will prove a decomposition result for the set $\operatorname{Conv}\left(P \cap F_{D}\right)$ (Theorem 3). The result states that $\operatorname{Conv}\left(P \cap F_{D}\right)$ can be written as the intersection of sets $\operatorname{Conv}\left(P(T) \cap F_{D}\right)$ over sets $T \in C_{1}^{*}$, where $C_{1}^{*}$ is a family of $(r+1)$-subsets of the constraints and $r$ is the rank of the constraint matrix $A$. Furthermore, when $r=n$, we show that it suffices to consider $r$-subsets of the constraints. Finally, we demonstrate how this result can be strengthened to obtain Theorem 1 for the split disjunctions $D\left(\pi, \pi_{0}^{1}, \pi_{0}^{2}\right)$.

We start by proving the following decomposition result on two-term disjunctions.

Theorem 4. Let $S \subseteq M$. If $S$ satisfies $|S| \geq r(S)+2$, then

$$
\operatorname{Conv}\left(P(S) \cap F_{D}\right)=\cap_{i \in S} \operatorname{Conv}\left(P(S \backslash\{i\}) \cap F_{D}\right) .
$$

Furthermore, (32) remains true if $r(S)=n$ and $|S|=n+1$. 
The following inclusion is obvious since $\operatorname{Conv}\left(P(S) \cap F_{D}\right) \subseteq \operatorname{Conv}\left(P(S \backslash\{i\}) \cap F_{D}\right)$.

Lemma 32. Let $S \subseteq M$ be non-empty.

$$
\operatorname{Conv}\left(P(S) \cap F_{D}\right) \subseteq \cap_{i \in S} \operatorname{Conv}\left(P(S \backslash\{i\}) \cap F_{D}\right) .
$$

However, the proof of the other inclusion involves the idea introduced by Balas [3] of lifting the set $\operatorname{Conv}\left(P(S) \cap F_{D}\right)$ into a higher dimensional space. Specifically, $\operatorname{Conv}(P(S) \cap$ $F_{D}$ ) can be described as the projection of the set, described by the constraints $(34)-(40)$, onto the space of $x$-variables (see [3]).

$$
\begin{array}{rlrl}
x & =x^{1}+x^{2}, & & \\
a_{i .}^{T} x^{1} & \leq b_{i} \lambda^{1}, & & \forall i \in S, \\
a_{i .}^{T} x^{2} & \leq b_{i} \lambda^{2}, & \forall i \in S, \\
\lambda^{1}+\lambda^{2} & =1, & & \\
D^{1} x^{1} & \leq d^{1} \lambda^{1}, & \\
D^{2} x^{2} & \leq d^{2} \lambda^{2}, & \\
\lambda^{1}, \lambda^{2} & \geq 0 .
\end{array}
$$

The description (34)-(40) can be projected into $\left(x, x^{1}, \lambda^{1}\right)$-space by using constraints (34) and (37). By doing this, we obtain the following characterization of $\operatorname{Conv}(P(S) \cap$ $\left.F_{D}\right)$.

$$
\begin{aligned}
-\lambda^{1} b_{i}+a_{i .}^{T} x^{1} & \leq 0, \quad \forall i \in S, \\
\lambda^{1} b_{i}-a_{i .}^{T} x^{1} & \leq b_{i}-a_{i .}^{T} x, \quad \forall i \in S, \\
\lambda^{1} & \leq 1, \\
-\lambda^{1} d^{1}+D^{1} x^{1} & \leq 0_{m_{1}}, \\
\lambda^{1} d^{2}-D^{2} x^{1} & \leq d^{2}-D^{2} x, \\
-\lambda^{1} & \leq 0 .
\end{aligned}
$$

The problem of deciding whether a given vector $x \in \mathbb{R}^{n}$ belongs to the set $\operatorname{Conv}(P(S)$ $\cap F_{D}$ ) can be decided by solving the following Phase I linear program called $P_{L P}(x, S)$, where the variables are $x^{1}, \lambda^{1}$ and $s$.

$$
\begin{aligned}
& \max -s \\
& -\lambda^{1} b_{i}+a_{i .}^{T} x^{1} \leq 0, \quad \forall i \in S, \quad\left(u_{i}\right) \\
& \lambda^{1} b_{i}-a_{i .}^{T} x^{1} \leq b_{i}-a_{i .}^{T} x, \quad \forall i \in S, \quad\left(v_{i}\right) \\
& \lambda^{1} \leq 1 \text {, } \\
& \left(w_{0}\right) \\
& -\lambda^{1} d^{1}+D^{1} x^{1}-s 1_{m_{1}} \leq 0_{m_{1}}, \quad\left(u^{0}\right) \\
& \lambda^{1} d^{2}-D^{2} x^{1}-s 1_{m_{2}} \leq d^{2}-D^{2} x, \quad\left(v^{0}\right) \\
& -\lambda^{1} \leq 0 \\
& -s \leq 0 . \quad\left(t_{1}\right)
\end{aligned}
$$


Observe that $P_{L P}(x, S)$ is feasible if and only if $x \in P(S)$. Furthermore $P_{L P}(x, S)$ is always bounded above by zero. Finally note that $x \in \operatorname{Conv}\left(P(S) \cap F_{D}\right)$ if and only if $P_{L P}(x, S)$ is feasible and bounded, and there exists an optimal solution in which the variable $s$ has the value zero.

Note that, in the remainder of this section, we will make extensive use of the dual $D_{L P}(x, S)$ of $P_{L P}(x, S)$. The name of the dual variable associated with each constraint in $P_{L P}(x, S)$ is given next to the constraints in (41) - (47). To prove the other direction of (32), we use the problem $P_{L P}(x, S)$ and its dual $D_{L P}(x, S)$. Specifically we use these problems on sets $S \subseteq M$ satisfying $|S| \geq 2$ and points $x \in P(S)$. Observe that the condition $x \in \cap_{i \in S} \operatorname{Conv}\left(P(S \backslash\{i\}) \cap F_{D}\right)$, where $|S| \geq 2$, implies $x \in P(S)$.

Throughout this section $\bar{x}$ denotes some element of $P(S)$. Let $\left(\bar{x}^{1}, \bar{\lambda}^{1}, \bar{s}\right)$ denote an optimal basic feasible solution to $P_{L P}(\bar{x}, S)$, and let $\left(\bar{u}, \bar{v}, \bar{u}^{0}, \bar{v}^{0}, \bar{w}_{0}, \bar{t}_{0}, \bar{t}_{1}\right)$ denote a corresponding optimal basic feasible solution to $D_{L P}(\bar{x}, S)$. Observe that the solutions to $P_{L P}(\bar{x}, S)$ and $D_{L P}(\bar{x}, S)$ depend on the values of $\bar{x}$ and $S$, i.e., when we write $\bar{u}_{i}$, say, we mean $\bar{u}_{i}(\bar{x}, S)$, where the values of $\bar{x}$ and $S$ should be clear from the context.

We now formulate the dual $D_{L P}(\bar{x}, S)$ of $P_{L P}(\bar{x}, S)$. The formulation uses the following quantities. Given $u^{0} \geq 0_{m_{1}}$ and scalars $u_{i} \geq 0$ for $i \in S$, define $\alpha^{1}\left(S, u, u^{0}\right):=$ $\sum_{i \in S} u_{i} a_{i}+\left(D^{1}\right)^{T} u^{0}$ and $\beta^{1}\left(S, u, u^{0}\right):=\sum_{i \in S} u_{i} b_{i}+\left(d^{1}\right)^{T} u^{0}$. Observe that the inequality $\left(\alpha^{1}\left(S, u, u^{0}\right)\right)^{T} x \leq \beta^{1}\left(S, u, u^{0}\right)$ is valid for the set $\left\{x \in P(S): D^{1} x \leq d^{1}\right\}$. Similarly, given $v^{0} \geq 0_{m_{2}}$ and scalars $v_{i} \geq 0$ for $i \in S$, defining the quantities $\alpha^{2}\left(S, v, v^{0}\right):=\sum_{i \in S} v_{i} a_{i}+\left(D^{2}\right)^{T} v^{0}$ and $\beta^{2}\left(S, v, v^{0}\right):=\sum_{i \in S} v_{i} b_{i}+\left(d^{2}\right)^{T} v^{0}$, gives the inequality $\left(\alpha^{2}\left(S, v, v^{0}\right)\right)^{T} x \leq \beta^{2}\left(S, v, v^{0}\right)$, which is valid for $\left\{x \in P(S): D^{2} x \leq\right.$ $\left.d^{2}\right\}$. With these quantities the dual $D_{L P}(\bar{x}, S)$ of $P_{L P}(\bar{x}, S)$ can be formulated as follows.

$\min$

$$
\begin{aligned}
\beta^{2}\left(S, v, v^{0}\right)-\left(\alpha^{2}\left(S, v, v^{0}\right)\right)^{T} \bar{x}+w_{0} & \\
\alpha^{1}\left(S, u, u^{0}\right)-\alpha^{2}\left(S, v, v^{0}\right) & =0, \\
\beta^{2}\left(S, v, v^{0}\right)-\beta^{1}\left(S, u, u^{0}\right)+w_{0}-t_{0} & =0, \\
1_{m_{1}}^{T} u^{0}+1_{m_{2}}^{T} v^{0}+t_{1} & =1, \\
u^{0} & \geq 0_{m_{1}}, \\
v^{0} & \geq 0_{m_{2}}, \\
w_{0}, t_{0}, t_{1} & \geq 0, \\
u_{i}, v_{i} & \geq 0,
\end{aligned}
$$

By our previous observation, $P_{L P}(x, S)$ is feasible if and only if $x \in P(S)$. We now characterize the optimal solution $\left(\bar{u}, \bar{v}, \bar{u}^{0}, \bar{v}^{0}, \bar{w}_{0}, \bar{t}_{0}, \bar{t}_{1}\right)$ to $D_{L P}(\bar{x}, S)$ for sets $S \subseteq M$ and points $\bar{x} \in P(S)$. First we consider the variables $u^{0}$ and $v^{0}$.

Lemma 33. Let $S \subseteq M$ be non-empty. Suppose $\bar{x} \in P(S) \backslash \operatorname{Conv}\left(P(S) \cap F_{D}\right)$. Then $\bar{u}^{0} \neq 0_{m_{1}}$ and $\bar{v}^{0} \neq 0_{m_{2}}$.

Proof. Let $S$ and $\bar{x}$ be as stated, and suppose firstly that $\bar{v}^{0}=0_{m_{2}}$. By the definition of $\alpha^{2}\left(S, v, v^{0}\right)$ and $\beta^{2}\left(S, v, v^{0}\right)$, the inequality $\left(\alpha^{2}\left(S, \bar{v}, 0_{m_{2}}\right)\right)^{T} x \leq \beta^{2}\left(S, \bar{v}, 0_{m_{2}}\right)$ is valid for $P(S)$. However the optimal objective value to $D_{L P}(\bar{x}, S)$ is negative (since $\bar{x} \notin$ $\left.\operatorname{Conv}\left(P(S) \cap F_{D}\right)\right)$, and $\bar{w}_{0} \geq 0$, so we must have $\beta^{2}\left(S, \bar{v}, 0_{m_{2}}\right)-\left(\alpha^{2}\left(S, \bar{v}, 0_{m_{2}}\right)\right)^{T} \bar{x}<$ 0 , a contradiction. 
Now suppose $\bar{u}_{0}=0_{m_{1}}$. The inequality $\left(\alpha^{1}\left(S, \bar{u}, 0_{m_{1}}\right)\right)^{T} x \leq \beta^{1}\left(S, \bar{u}, 0_{m_{1}}\right)$ is valid for $P(S)$, but $\beta^{1}\left(S, \bar{u}, 0_{m_{1}}\right)-\left(\alpha^{1}\left(S, \bar{u}, 0_{m_{1}}\right)\right)^{T} \bar{x} \leq \beta^{2}\left(S, \bar{v}, \bar{v}^{0}\right)+w_{0}-$ $\left(\alpha^{2}\left(S, \bar{v}, \bar{v}^{0}\right)\right)^{T} \bar{x}<0$, (from (48) and (49) above). This contradicts $\bar{x} \in P(S)$.

Observe that Lemma 33 implies that at least two of the variables in $\left(u^{0}, v^{0}\right)$ are basic when $\bar{x} \in P(S) \backslash \operatorname{Conv}\left(P(S) \cap F_{D}\right)$.

Define $B_{u}:=\left\{i \in S: u_{i}\right.$ basic $\}$ and $B_{v}:=\left\{i \in S: v_{i}\right.$ basic $\}$ to be the set of basic $u$ 's and $v$ 's respectively. The next lemma describes properties of the variables $u_{i}$ and $v_{i}$ for $i \in S$ when $\bar{x}$ belongs to $\cap_{i \in T} \operatorname{Conv}\left(P(S \backslash\{i\}) \cap F_{D}\right)$, but not to $\operatorname{Conv}\left(P(S) \cap F_{D}\right)$, where $T$ is some subset of $S$ of size at least two.

Lemma 34. Suppose $\bar{x} \in \cap_{i \in T} \operatorname{Conv}\left(P(S \backslash\{i\}) \cap F_{D}\right)$ and $\bar{x} \notin \operatorname{Conv}\left(P(S) \cap F_{D}\right)$, where $T \subseteq S \subseteq M$ and $|T| \geq 2$. Let $B_{u}(T):=B_{u} \cap T$ and $B_{v}(T):=B_{v} \cap T$, i.e., $B_{u}(T)$ and $B_{v}(T)$ denote the basic $u$ 's and $v$ 's in the set $T$ respectively. Then

(i) For each $i \in T$, either $u_{i}$ or $v_{i}$ has a positive value, i.e., $\bar{u}_{i}>0$ or $\bar{v}_{i}>0$ for all $i \in T$.

(ii) $B_{u}(T) \cap B_{v}(T)=\emptyset$ and $B_{u}(T) \cup B_{v}(T)=T$, i.e., exactly one of the variables $u_{i}$ and $v_{i}$ is basic for all $i \in T$.

(iii) The vectors $\left\{\left[a_{i}^{T},-b_{i}\right]\right\}_{i \in T}$ are linearly independent.

(iv) $\left|B_{u}(T) \cup B_{v}(T)\right|=|T| \leq r(T)+1$, i.e., the size of $T$ is bounded by $r(T)+1$.

Proof. Let $S, T$ and $\bar{x}$ be as stated. We will prove (i) first. Suppose by contradiction $\bar{u}_{i^{\prime}}=0$ and $\bar{v}_{i^{\prime}}=0$ for some $i^{\prime} \in T$. Since $\bar{x} \notin \operatorname{Conv}\left(P(S) \cap F_{D}\right)$, the linear program $P_{L P}(\bar{x}, S)$ is infeasible, and hence its dual $D_{L P}(\bar{x}, S)$ is unbounded. Because $\bar{u}_{i^{\prime}}=0$ and $\bar{v}_{i^{\prime}}=0$, the linear program $D_{L P}^{\prime}\left(\bar{x}, S \backslash\left\{i^{\prime}\right\}\right)$, obtained from $D_{L P}(\bar{x}, S)$ by eliminating $u_{i^{\prime}}, v_{i^{\prime}}$ and the normalization constraint (50), is also unbounded. Consequently, the dual of $D_{L P}^{\prime}\left(\bar{x}, S \backslash\left\{i^{\prime}\right\}\right)$, the problem $P_{L P}^{\prime}\left(\bar{x}, S \backslash\left\{i^{\prime}\right\}\right)$, is infeasible. But $P_{L P}^{\prime}\left(\bar{x}, S \backslash\left\{i^{\prime}\right\}\right)$ can be obtained from $P_{L P}(\bar{x}, S)$ by eliminating the variable $s$ and the constraints corresponding to $i^{\prime}$ in (41) and (42), and we see that $P_{L P}^{\prime}\left(\bar{x}, S \backslash\left\{i^{\prime}\right\}\right)$ is feasible if and only if $\bar{x}$ is in $\operatorname{Conv}\left(P\left(S \backslash\left\{i^{\prime}\right\}\right) \cap F_{D}\right)$. The fact that $\bar{x} \in \operatorname{Conv}\left(P\left(S \backslash\left\{i^{\prime}\right\}\right) \cap F_{D}\right)$ implies the feasibility of $P_{L P}^{\prime}\left(\bar{x}, S \backslash\left\{i^{\prime}\right\}\right)$, which is a contradiction. Hence $\bar{u}_{i}>0$ or $\bar{v}_{i}>0$ for all $i \in T$, which gives $B_{u}(T) \cup B_{v}(T)=T$. This proves (i). To prove (ii), we show that at most one of $u_{i}$ and $v_{i}$ can be basic.

The feasible set for $D_{L P}(\bar{x}, S)$ can be written in the form $\left\{y \in \mathbb{R}^{n^{\prime}}: Z y=z^{0}, y \geq\right.$ $\left.0_{n^{\prime}}\right\}$, where $Z$ and $z^{0}$ are of suitable dimensions. Let $i \in T$ be arbitrary. The column of $Z$ corresponding to $u_{i}$ is given by $\left[a_{i}^{T},-b_{i}, 0\right]^{T}$. Similarly the column of $Z$ corresponding to $v_{i}$ is given by $\left[-a_{i}^{T}, b_{i}, 0\right]^{T}$. Hence the columns of $Z$ corresponding to $u_{i}$ and $v_{i}$ are linearly dependent. Since the columns of $Z$ corresponding to basic variables must be linearly independent, it follows that at most one of $u_{i}$ and $v_{i}$ can be basic. This also shows that the vectors $\left\{\left[a_{i}^{T},-b_{i}\right]\right\}_{i \in T}$ are linearly independent. This completes the proof of (i), (ii) and (iii).

Finally, since the vectors $\left\{\left[-a_{i}^{T}, b_{i}\right]\right\}_{i \in T}$ are linearly independent, there must exist a set $T^{\prime} \subseteq T$ of cardinality either $|T|$ or $|T|-1$ such that the vectors $\left\{a_{i}\right\}_{i \in T^{\prime}}$ are linearly independent. It follows that $\left|B_{u}(T) \cup B_{v}(T)\right|=|T| \leq r(T)+1$. Therefore (iv) is also proved.

With the above lemmas we can prove the missing direction in Theorem 4. 
Lemma 35. Let $S \subseteq M$, and suppose that either $|S| \geq r(S)+2$ or $r(S)=n$ and $|S|=n+1$. Then

$$
\operatorname{Conv}\left(P(S) \cap F_{D}\right) \supseteq \cap_{i \in S} \operatorname{Conv}\left(P(S \backslash\{i\}) \cap F_{D}\right) .
$$

Proof. Let $\bar{x} \in \cap_{i \in S} \operatorname{Conv}\left(P(S \backslash\{i\}) \cap F_{D}\right)$ and suppose by contradiction $\bar{x} \notin \operatorname{Conv}\left(P(S) \cap F_{D}\right)$. If $|S| \geq r(S)+2$, Lemma 34(iv) with $T=S$ implies that $S \leq r(S)+1$, a contradiction. If $|S|=n+1$, since the number of basic variables is bounded by the number of equality constraints in $D_{L P}(\bar{x}, S)$, the number of basic variables in the solution $\left(\bar{u}, \bar{v}, \bar{u}^{0}, \bar{v}^{0}, \bar{w}_{0}, \bar{t}_{0}, \bar{t}_{1}\right)$ is at most $n+2$. The number of basic variables among the variables $u_{i}$ and $v_{i}$ for $i \in S$ is $|S|=n+1$ (Lemma 34 with $T:=S)$. However, according to Lemma 33, at least two of the variables in $\left(\bar{u}^{0}, \bar{v}^{0}\right)$ are basic, which gives a total of $n+3$ basic variables, a contradiction.

We now strengthen Theorem 4 for the case where $|S| \geq r(S)+2$. Let $\bar{I}(S)$ be the set of constraints $i \in S$ whose removal from $S$ leaves the rank unchanged, i.e., $\bar{I}(S):=\{i \in S: r(S)=r(S \backslash\{i\})\}$. We have

Theorem 5. Let $S \subseteq M$ satisfy $|S| \geq r(S)+2$. Then

$$
\operatorname{Conv}\left(P(S) \cap F_{D}\right)=\cap_{i \in \bar{I}(S)} \operatorname{Conv}\left(P(S \backslash\{i\}) \cap F_{D}\right) .
$$

Like in Theorem 4, and with the a similar proof, the inclusion " $\subseteq$ " of Theorem 5 is easy to prove. To prove the remaining inclusion, we observe that the condition $|S| \geq r(S)+1$ and the assumption $a_{i} \neq 0_{n}, \forall i \in M$, imply $|\bar{I}(S)| \geq 2$. Therefore, $\cap_{i \in \bar{I}(S)} \operatorname{Conv}\left(P(S \backslash\{i\}) \cap F_{D}\right) \subseteq P(S)$, so that the problem $P_{L P}(\bar{x}, S)$ is feasible and bounded for a point $\bar{x} \in \cap_{i \in \bar{I}(S)} \operatorname{Conv}\left(P(S \backslash\{i\}) \cap F_{D}\right)$. The following lemma gives a formal statement and proof of the remaining inclusion.

Lemma 36. Let $S \subseteq M$ satisfy $|S| \geq r(S)+2$. Then

$$
\operatorname{Conv}\left(P(S) \cap F_{D}\right) \supseteq \cap_{i \in \bar{I}(S)} \operatorname{Conv}\left(P(S \backslash\{i\}) \cap F_{D}\right) .
$$

Proof. Let $\bar{x} \in \cap_{i \in \bar{I}(S)} \operatorname{Conv}\left(P(S \backslash\{i\}) \cap F_{D}\right)$ and assume by contradiction $\bar{x} \notin$ $\operatorname{Conv}\left(P(S) \cap F_{D}\right)$. Observe that it suffices to prove that the vectors $\left\{\left[a_{i}^{T},-b_{i}\right]\right\}_{i \in S}$ are linearly independent, since that contradicts $|S| \geq r(S)+2$. Assume for a contradiction that the vectors $\left\{\left[a_{i}^{T},-b_{i}\right]\right\}_{i \in S}$ are linearly dependent, and let $k \in S$ satisfy $\left[a_{k}^{T},-b_{k}\right]=\sum_{i \in S \backslash\{k\}} \mu_{i}\left[a_{i}^{T},-b_{i}\right]$, where $\left\{\mu_{i}\right\}_{i \in S \backslash\{k\}}$ are not all zero. This means $a_{k} \in \operatorname{Span}\left(\left\{a_{i}\right\}_{i \in S \backslash\{k\}}\right)$, which implies $r(S)=r(S \backslash\{k\})$, i.e., $k \in \bar{I}(S)$.

We have shown that, if $\left[a_{k}^{T},-b_{k}\right]=\sum_{i \in S \backslash\{k\}} \mu_{i}\left[a_{i .}^{T},-b_{i}\right]$, where $k \in S$ and $\left\{\mu_{i}\right\}_{i \in S \backslash\{k\}}$ are not all zero, then $k \in \bar{I}(S)$. Note that this implies $\mu_{i}=0$ for all $i \in$ $S \backslash \bar{I}(S)$. To see this, assume for a contradiction that $\mu_{l} \neq 0$ for some $l \in S \backslash \bar{I}(S)$. Then we may write $\left[a_{l .}^{T},-b_{l}\right]=\sum_{i \in S \backslash\{l\}} \mu_{i}^{\prime}\left[a_{i}^{T},-b_{i}\right]$, where $\mu_{i}^{\prime}=\frac{\mu_{i}}{\mu_{l}}$ for $i \in S \backslash\{k, l\}$ and $\mu_{k}^{\prime}=\frac{1}{\mu_{l}}$. As observed above, this implies $l \in \bar{I}(S)$, a contradiction. It follows that $\mu_{i}=0$ for all $i \in S \backslash \bar{I}(S)$.

We therefore have $\left[a_{k}^{T},-b_{k}\right]=\sum_{i \in \bar{I}(S) \backslash\{k\}} \mu_{i}\left[a_{i}^{T},-b_{i}\right]$ and $k \in \bar{I}(S)$. However, according to Lemma 34(iii) with $T:=\bar{I}(S)$, the vectors $\left\{\left[a_{i .}^{T},-b_{i}\right]\right\}_{i \in \bar{I}(S)}$ are linearly independent, a contradiction. 
If we apply Theorem 5 iteratively to sets $S$ satisfying $|S| \geq r(S)+2$, and Theorem 4 for sets $S$ satisfying $|S|=n+1$, we obtain Theorem 3 in Sect. 2. The example in Figure 4 in Sect. 2 demonstrates that the assumption $|S| \geq r(S)+2$ is necessary for (57) to be true.

In the following we prove that (57) remains valid for $|S|=r(S)+1$ for the special case of a general split disjunction $D\left(\pi, \pi_{0}^{1}, \pi_{0}^{2}\right)$, where $\pi_{0}^{1}<\pi_{0}^{2}$. The problem $P_{L P}(\bar{x}, S)$ for the disjunction $D\left(\pi, \pi_{0}^{1}, \pi_{0}^{2}\right)$, which will be called $P_{L P}^{S}(\bar{x}, S)$ in the following, is obtained from the problem $P_{L P}(\bar{x}, S)$ by replacing (44) and (45) with

$$
\begin{array}{ll}
-\lambda^{1} \pi_{0}^{1}+\pi^{T} x^{1}-s \leq 0, & \left(u^{0}\right) \\
-\lambda^{1} \pi_{0}^{2}+\pi^{T} x^{1}-s \leq-\pi_{0}^{2}+\pi^{T} \bar{x} . & \left(v^{0}\right)
\end{array}
$$

The dual of $P_{L P}^{S}(\bar{x}, S)$ is the problem $D_{L P}^{S}(\bar{x}, S)$ as follows.

$$
\begin{aligned}
\min \quad \sum_{i \in S} v_{i}\left(b_{i}-a_{i .}^{T} \bar{x}\right)+w_{0}+v^{0}\left(\pi^{T} \bar{x}-\pi_{0}^{2}\right) & \\
\text { s.t. } \quad \sum_{i \in S} a_{i .}\left(u_{i}-v_{i}\right)+\pi\left(u^{0}+v^{0}\right) & =0_{n}, \\
\sum_{i \in S} b_{i}\left(v_{i}-u_{i}\right)-\pi_{0}^{1} u^{0}-\pi_{0}^{2} v^{0}+w_{0}-t_{0} & =0 \\
u^{0}+v^{0}+t_{1} & =1 \\
u^{0}, v^{0}, w_{0}, t_{0}, t_{1} & \geq 0 \\
u_{i}, v_{i} & \geq 0, \quad \forall i \in S .
\end{aligned}
$$

The optimal solution $\left(\bar{u}, \bar{v}, \bar{u}^{0}, \bar{v}^{0}, \bar{w}_{0}, \bar{t}_{0}, \bar{t}_{1}\right)$ to $D_{L P}^{S}(\bar{x}, S)$, for the case where $|S|=$ $r(S)+1$, is characterized in the following lemma (also see Lemma 2 in [4]). The statements of this lemma are very similar to the statements made in Lemma 34. However, the assumptions are different than in Lemma 34. Also, we now prove that the vectors $\left\{a_{i}\right\}_{i \in B_{u} \cup B_{v}}$ are linearly independent, and this does not follow from the statement of Lemma 34 that the vectors $\left\{\left[a_{i},-b_{i}\right]\right\}_{i \in B_{u} \cup B_{v}}$ are linearly independent.

Lemma 37. Suppose that $\bar{x} \in \cap_{i \in \bar{I}(S)} \operatorname{Conv}\left(P(S \backslash\{i\}) \cap F_{D\left(\pi, \pi_{0}^{1}, \pi_{0}^{2}\right)}\right.$ and $\bar{x} \notin \operatorname{Conv}\left(P(S) \cap F_{D\left(\pi, \pi_{0}^{1}, \pi_{0}^{2}\right)}\right)$. Then $B_{u} \cap B_{v}=\emptyset, r(S)=n,\left|B_{u} \cup B_{v}\right|=n$, and the vectors $\left\{a_{i .}\right\}_{i \in B_{u} \cup B_{v}}$ are linearly independent.

Proof. The feasible set for $D_{L P}^{S}(\bar{x}, S)$ can be written in the form $\left\{y \in \mathbb{R}^{n^{\prime}}: Z y=\right.$ $\left.z_{0}, y \geq 0_{n^{\prime}}\right\}$, where $Z$ and $z_{0}$ are of suitable dimensions. We first argue that the variables $w_{0}, t_{0}$ and $t_{1}$ take the value 0 . Since $\bar{x} \notin \operatorname{Conv}\left(P(S) \cap F_{D\left(\pi, \pi_{0}^{1}, \pi_{0}^{2}\right)}\right)$, the optimal objective value of $P_{L P}^{S}(\bar{x}, S)$ and $D_{L P}^{S}(\bar{x}, S)$ is $-\bar{s}<0$, i.e., $s$ is basic. By complementarity slackness, we deduce that $t_{1}=0$. From Lemma 33 it follows that both $u^{0}$ and $v^{0}$ are basic. The column in $Z$ corresponding to $u^{0}$ is $\left[\pi^{T},-\pi_{0}^{1}, 1\right]^{T}$ and the column corresponding to $v^{0}$ is $\left[\pi^{T},-\pi_{0}^{2}, 1\right]^{T}$. Subtracting the column corresponding to $v^{0}$ from the column corresponding to $u^{0}$ gives a constant times $e_{n+1}$, i.e., a constant times the $(n+1)^{\text {th }}$ unit vector in $\mathbb{R}^{n+2}$. Since the column corresponding to both $w_{0}$ and $-t_{0}$ is $e_{n+1}$, and since basic columns are linearly independent, $w_{0}$ and $t_{0}$ must be non-basic. Therefore, both $w_{0}$ and $t_{0}$ take the value 0 . 
For all $i \in S$, not both $v_{i}$ and $u_{i}$ can be in the basis, since their corresponding columns in $Z$ are multiples of each other. Hence $B_{u} \cap B_{v}=\varnothing$. Furthermore, since $\left(\bar{u}, \bar{v}, \bar{u}^{0}, \bar{v}^{0}, \bar{w}_{0}, \bar{t}_{0}, \bar{t}_{1}\right)$ is a basic solution to $D_{L P}^{S}(\bar{x}, S)$, the following system has a unique solution.

$$
\begin{array}{lr}
\sum_{i \in B_{u}} a_{i .} u_{i}-\sum_{i \in B_{v}} a_{i .} v_{i}+\pi\left(u^{0}+v^{0}\right)=0_{n} \\
\sum_{i \in B_{v}} b_{i} v_{i}-\sum_{i \in B_{u}} b_{i} u_{i}-\pi_{0}^{1} u^{0}-v^{0} \pi_{0}^{2}=0 \\
u^{0}+v^{0} & =1
\end{array}
$$

The system (65)-(67) is of the form $Z^{B} y=z_{0}^{B}$. The number of rows of $Z^{B}$ (and $z_{0}^{B}$ ) is $n+2$ and the number of columns is $\left|B_{u} \cup B_{v}\right|+2$. All columns of $Z^{B}$ are linearly independent. Therefore we must have $\left|B_{u} \cup B_{v}\right|=n$, since otherwise multiple solutions would exist.

Now we show that the vectors $\left\{a_{i .}\right\}_{i \in B_{u} \cup B_{v}}$ are linearly independent. Then $r(S)=$ $n$ is also proved. Assume by contradiction that the vectors $\left\{a_{i}\right\}_{i \in B_{u} \cup B_{v}}$ are linearly dependent. Then there exists a non-zero solution $\left(u^{*}, v^{*}\right)$ to the system $\sum_{i \in B_{u}} a_{i .} u_{i}^{*}-$ $\sum_{i \in B_{v}} a_{i} v_{i}^{*}=0_{n}$. Define scalars $u_{i}(\delta):=\bar{u}_{i}+\delta u_{i}^{*}$ for $i \in B_{u}$ and $v_{i}(\delta):=\bar{v}_{i}+$ $\delta v_{i}^{*}$ for $i \in B_{v}$, where $\delta \in \mathbb{R}$. By solving (65)-(67) for the two remaining variables, we get that $\left(u(\delta), v(\delta), u^{0}(\delta), v^{0}(\delta)\right)$ satisfies (65)-(67) if and only if $u^{0}(\delta)=1-$ $v^{0}(\delta)$ and $v^{0}(\delta)=\left(\pi_{0}^{1} \bar{u}_{0}+\pi_{0}^{2} \bar{v}_{0}-\pi_{0}^{1}+\delta\left(\sum_{i \in B_{v}} b_{i} v_{i}^{*}-\sum_{i \in B_{u}} b_{i} u_{i}^{*}\right)\right) /\left(\pi_{0}^{2}-\pi_{0}^{1}\right)$. Since the numbers $\left\{u_{i}^{*}\right\}_{i \in B_{u}} \cup\left\{v_{i}^{*}\right\}_{i \in B_{v}}$ are not all zero there must exist $\delta^{*} \in \mathbb{R}$ such that $\left(u\left(\delta^{*}\right), v\left(\delta^{*}\right), u^{0}\left(\delta^{*}\right), v^{0}\left(\delta^{*}\right)\right)$ is a different solution to (65)-(67) than $\left(\bar{u}, \bar{v}, \bar{u}_{0}, \bar{v}_{0}\right)$, which is a contradiction.

From the above lemma we have the desired extension of Theorem 4 for split disjunctions.

Lemma 38. Suppose $S \subseteq M$ satisfies $|S|=r(S)+1$. Then

$$
\operatorname{Conv}\left(P(S) \cap F_{D\left(\pi, \pi_{0}^{1}, \pi_{0}^{2}\right)}\right)=\cap_{i \in \bar{I}(S)} \operatorname{Conv}\left(P(S \backslash\{i\}) \cap F_{D\left(\pi, \pi_{0}^{1}, \pi_{0}^{2}\right)}\right) .
$$

Proof. By a similar argument to the proof of Lemma 11, it is easy to show the inclusion $\operatorname{Conv}\left(P(S) \cap F_{D\left(\pi, \pi_{0}^{1}, \pi_{0}^{2}\right)}\right) \subseteq \cap_{i \in \bar{I}(S)} \operatorname{Conv}\left(P(S \backslash\{i\}) \cap F_{D\left(\pi, \pi_{0}^{1}, \pi_{0}^{2}\right)}\right)$. We prove the remaining inclusion by contradiction. Suppose we have $\bar{x} \in \cap_{i \in \bar{I}(S)} \operatorname{Conv}(P(S \backslash$ $\left.\{i\}) \cap F_{D\left(\pi, \pi_{0}^{1}, \pi_{0}^{2}\right)}\right)$ and $\bar{x} \notin \operatorname{Conv}\left(P(S) \cap F_{D\left(\pi, \pi_{0}^{1}, \pi_{0}^{2}\right)}\right.$. By Lemma 37, $B_{u} \cap B_{v}=\emptyset$, $r(S)=n,\left|B_{u} \cup B_{v}\right|=n$, and the vectors $\left\{a_{i}\right\}_{i \in B_{u} \cup B_{v}}$ are linearly independent. Let $\{\bar{i}\}=S \backslash\left(B_{u} \cup B_{v}\right)$. We have that $\bar{i} \notin \bar{I}(S)$, since otherwise Lemma 34(i) with $T:=\bar{I}(S)$ would imply $\bar{u}_{\bar{i}}>0$ or $\bar{v}_{\bar{i}}^{-}>0$, which contradicts $\bar{i} \notin B_{u} \cup B_{v}$. But $\bar{i} \in S \backslash \bar{I}(S)$ implies that $\bar{i}$ is in every basis of $\mathrm{S}$, which contradicts $\bar{i} \notin B_{u} \cup B_{v}$.

From Theorem 5 and Lemma 38, we get Theorem 1 of Sect. 2.

Acknowledgements. The authors are grateful to the referees for their detailed and valuable comments, which have helped in improving the presentation of this paper. Special thanks to Jean-Philippe Richard for his careful reading and extensive reports. 


\section{References}

1. Andersen, K., Cornuéjols, G., Li, Y.: Reduce-and-split cuts: Improving the performance of mixed integer Gomory cuts. Submitted for publication, 2004

2. Balas, E.: Intersection cuts - a new type of cutting planes for integer programming. Oper. Res. 19, 19-39 (1971)

3. Balas, E.: Disjunctive programming. Ann. Discrete Math. 5, 3-51 (1979)

4. Balas, E., Perregaard, M.: A Precise correspondence between, lift-and-project cuts, simple disjunctive cuts and mixed integer Gomory cuts for 0-1 programming. Mathematical Programming B 94, 221-245 (2003)

5. Bixby, R.E., Gu, Z., Rothberg, E., Wunderling, R.: Mixed integer programming: a progress report. In: M. Grötschel (ed)., The sharpest cut: the impact of Manfred Padberg and his work, MPS/SIAM Series on Optimization 2004, pp. 309-326

6. Cook, W., Kannan, R., Schrijver, A.: Chvátal closures for mixed integer programs. Mathematical Programming 47, 155-174 (1990)

7. Cornuéjols, G., Li, Y.: Elementary closures for integer programs. Oper. Res. Lett. 28, 1-8 (2001)

8. Cornuéjols, G., Li, Y.: On the rank of mixed 0, 1 polyhedra. Mathematical Programming 91, 391-397 (2002)

9. Gomory, R.: An algorithm for the mixed integer problem. Techinical Report RM-2597, The Rand Coporation, 1960

10. Nemhauser, G., Wolsey, L.: A recursive procedure to generate all cuts for 0-1 mixed integer programs. Mathematical Programming 46, 379-390 (1990) 